\title{
WestVirginiaUniversity
}

THE RESEARCH REPOSITORY @ WVU

Graduate Theses, Dissertations, and Problem Reports

2011

\section{Vegetative compatibility in arbuscular mycorrhizal fungi}

Sonia Purin

West Virginia University

Follow this and additional works at: https://researchrepository.wvu.edu/etd

\section{Recommended Citation}

Purin, Sonia, "Vegetative compatibility in arbuscular mycorrhizal fungi" (2011). Graduate Theses,

Dissertations, and Problem Reports. 4769.

https://researchrepository.wvu.edu/etd/4769

This Dissertation is protected by copyright and/or related rights. It has been brought to you by the The Research Repository @ WVU with permission from the rights-holder(s). You are free to use this Dissertation in any way that is permitted by the copyright and related rights legislation that applies to your use. For other uses you must obtain permission from the rights-holder(s) directly, unless additional rights are indicated by a Creative Commons license in the record and/ or on the work itself. This Dissertation has been accepted for inclusion in WVU Graduate Theses, Dissertations, and Problem Reports collection by an authorized administrator of The Research Repository @ WVU.

For more information, please contact researchrepository@mail.wvu.edu. 


\title{
VEGETATIVE COMPATIBILITY IN ARBUSCULAR MYCORRHIZAL FUNGI
}

\author{
Sonia Purin
}

\section{Dissertation submitted to the Davis College of Agriculture, Natural Resources and Design at West Virginia University in partial fulfillment of the requirements for the degree of}

Doctor of Philosophy in Agricultural Sciences

Joseph B. Morton, Ph.D., Chair

Alan J. Sexstone, Ph.D. Daniel G. Panaccione, Ph.D. James D. Bever, Ph.D. Teresa E. Pawlowska, Ph.D.

Division of Plant and Soil Sciences

Morgantown, WV

2011

Keywords: vegetative compatibility, anastomosis, arbuscular mycorrhizal fungi

Copyright 2011 Sonia Purin 


\section{ABSTRACT \\ VEGETATIVE COMPATIBILITY IN ARBUSCULAR MYCORRHIZAL FUNGI}

Sonia Purin

The process of anastomosis promotes interconnectivity between hyphal branches during formation of a mycelial network and between fungal colonies. Self anastomosis involves fusion within a mycelium from a germinating spore, and this process is common in all species of fungi. Non-self anastomosis involves fusion between hyphae from different spores or colonies and allows lateral gene transfer, but this process is much rarer. Anastomosis in Ascomycota is well studied, but little is known for asexual arbuscular mycorrhizal fungi (AMF) in Glomeromycota. Because AMF are obligate symbionts in plant roots, studies have focused on anastomosis in asymbiotic hyphae of germinating spores. To study behavior of symbiotic hyphae growing from a mycorrhizal plant, a "rhizohyphatron" was designed which allowed symbiotic hyphae to grow from single or paired containerized mycorrhizal plants onto an agar-coated surface that could be removed and examined microscopically. In Chapter 1, anastomosis was studied in genetically uncharacterized populations of AMF species representing major genera. Hyphal fusions occurred at very low frequencies in Glomus clarum and Glomus intraradices $(<15 \%)$ but were absent in Ambispora, Gigaspora, Paraglomus and Scutellospora. These results suggest that anastomosis in symbiotic mycelia may not be biologically important for most clades of AMF. In Chapter 2, self and non-self anastomosis were studied in both asymbiotic and symbiotic hyphae of G. clarum from single spore cultures genotyped using two anonymous microsatellite-flanking markers. Frequency of self anastomosis was similar in asymbiotic hyphae from germinating spores and symbiotic mycelia from plants, ranging from 8 to $38 \%$. Non-self anastomosis was observed only in asymbiotic hyphae of isolates that were genetically identical as well as different isolates. However, fusions occurred in less than $6 \%$ of hyphal contacts. Such low levels of hyphal fusion may still permit gene flow in AMF populations, but anastomosis is restricted to asymbiotic hyphae of isolates from proximate locations. Results from this study show that vegetative compatibility between individuals occurs in AMF, but it is limited to only a few lineages and is constrained to only a small phase of the AMF life cycle. 


\section{Dedication}

God has made these verses become true in my life as never before:

"The Lord is my shepherd; I have everything I need. He lets me rest in fields of green grass and leads me to quiet pools of fresh water. He gives me new strength. He guides me in the right paths, as he has promised. Even if I go through the deepest darkness, I will not be afraid, Lord, for you are with me".

Psalms 23: 1-4.

I truly had everything I needed, and I always will. 


\section{Acknowledgements}

Most importantly, I would like to thank God. His grace has been my source of strength, peace, love and safety. My faith in Him has made this accomplishment possible. Day after day, he has poured out countless blessings and fulfilled my every physical and spiritual need.

I would like to honor my parents, Evanilde and João, for their love. I lost my lovely mother before I started my Ph.D. program, but I believe she would be very happy for this accomplishment if she were still with me.

I want to thank my fiancé, Rob Bills. From picking spores, studying biochemistry, coaching a boy's basketball team, and bird watching; we have shared many wonderful experiences together. I want to thank him for his unconditional love, endless support, and for always being at my side.

I am especially thankful to Rick and Debbie Mullens for "adopting" me and making me a part of their family. They are a blessing and wonderful example of love, friendship, parenthood and discipleship. I will never forget their kindness, generosity and support.

I wish to thank Pastor Casey Shorr and my brothers in Christ from the Pierpont Church of the Nazarene. I can't thank them enough for their companionship and for making me feel at home in this loving community.

I am particularly thankful to my friends Marciela de Souza, Carlos Grippa, Rosane Giacomozzi, Angela Purim and Lourdes Araujo. They have proved to me that true friendship can overcome both distance and time. I am very blessed to have them in my life.

I would like to express my gratefulness to my advisor, Dr. Joseph Morton. His guidance, encouragement and support were essential for this accomplishment. He also supported me financially throughout this program and provided me an assistantship for the last year, and I am grateful for that. I am also extremely thankful for the mentorship received from my committee members: Alan Sexstone, Daniel Panaccione, James Bever and Teresa Pawlowska. A special thank you goes to Dr. Bever and Dr. Pawlowska who always managed their schedule to accommodate my needs in Morgantown. I really appreciate their participation, encouragement and support.

I would like to thank my friend and former lab mate Zola Msiska. Much of my experience with molecular biology comes from her.

I would like to extend my appreciation to Bill Wheeler. Every student who has been in Morton's lab knows that we cannot succeed without him. He is truly our superman.

This Ph.D. program was financially supported by the Brazilian Federal Government (CAPES) and the Fulbright Program. I would like to thank both institutions for this exceptional scholarship, and for the unique opportunity to experience graduate education and life in the United States. 


\section{TABLE OF CONTENTS}

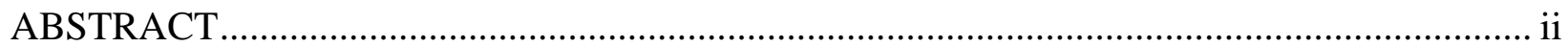

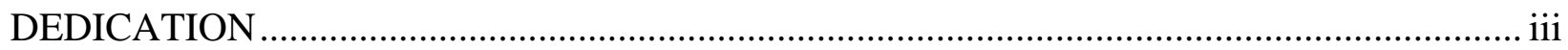

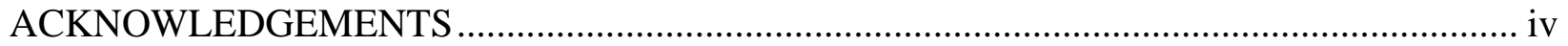

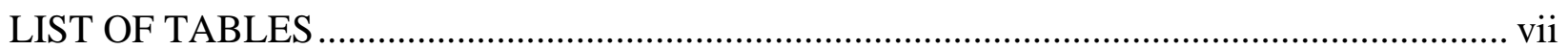

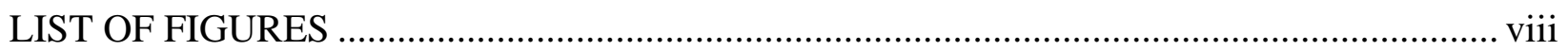

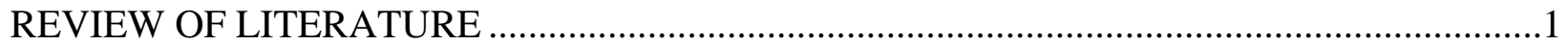

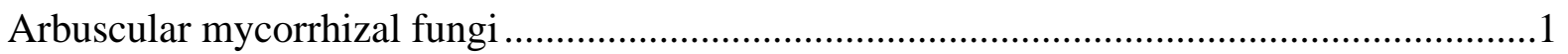

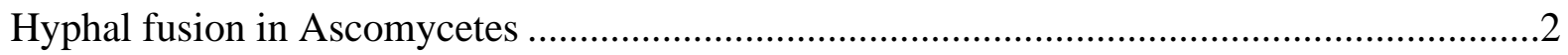

Incompatibility systems in Podospora anserina ..............................................................4

Incompatibility systems in Neurospora crassa .........................................................

Occurrence and significance of anastomosis in Ascomycetes..........................................5

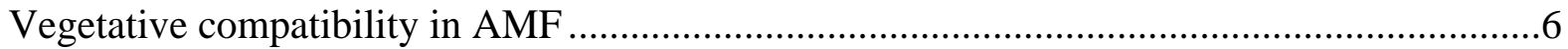

Vegetative compatibility within populations ..................................................................

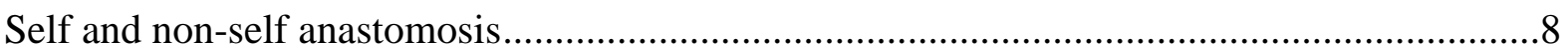

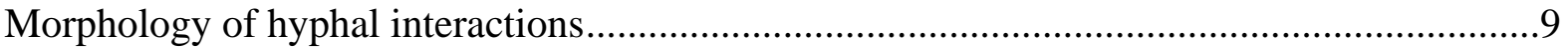

Significance of anastomosis in arbuscular mycorrhizal fungi .......................................... 9

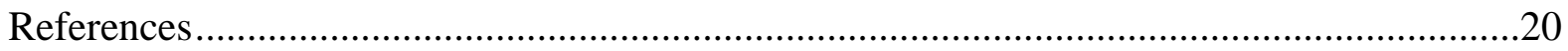

CHAPTER 1. IN SITU ANALYSIS OF ANASTOMOSIS IN REPRESENTATIVE

GENERA OF ARBUSCULAR MYCORRHIZAL FUNGI .................................................25

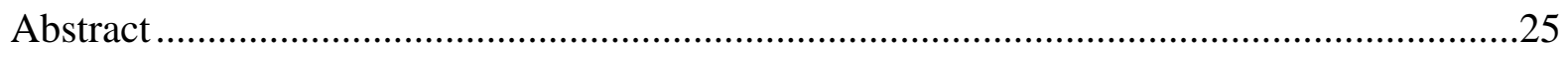

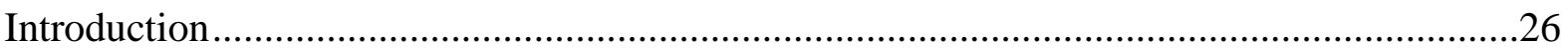

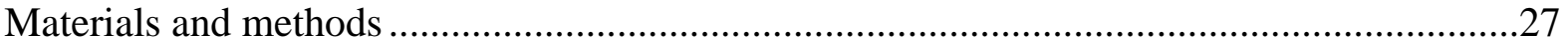

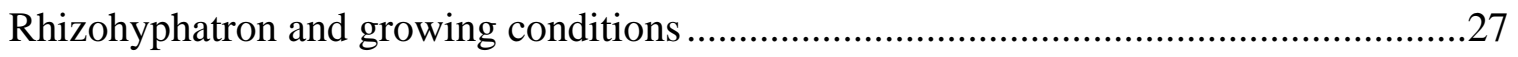

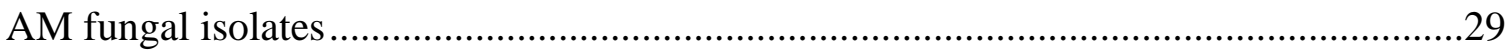

Microscopic evaluation of hyphal contacts ...........................................................29

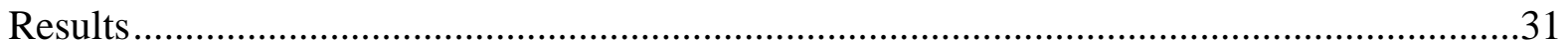

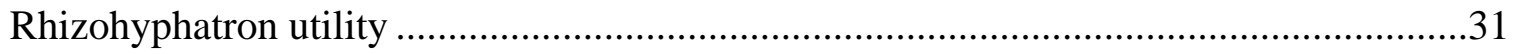

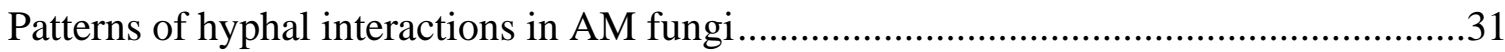

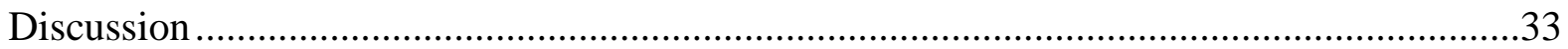




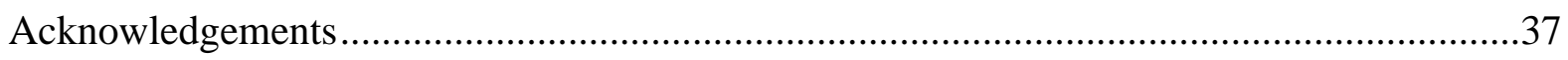

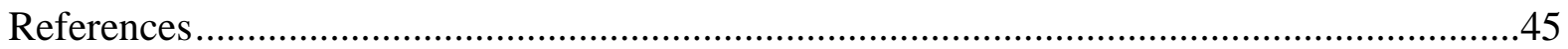

CHAPTER 2. ASYMBIOTIC AND SYMBIOTIC HYPHAE OF THE ARBUSCULAR MYCORRHIZAL FUNGUS GLOMUS CLARUM HAVE UNIQUE ROLES IN NON-SELF

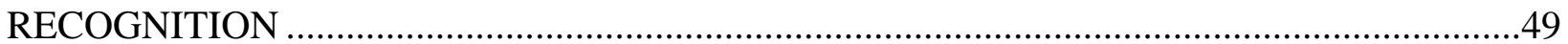

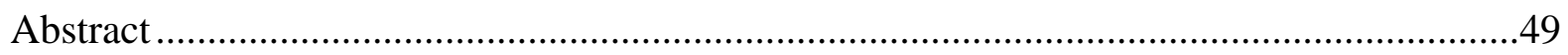

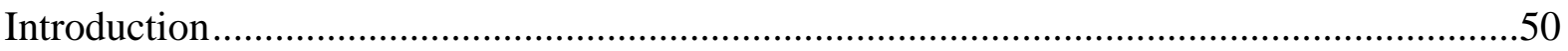

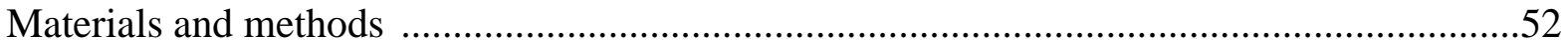

Sampling sites and establishment of single spore cultures .............................................52

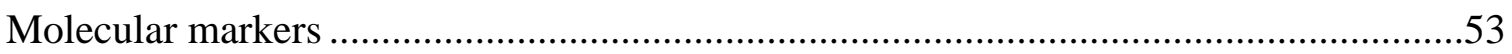

Genotyping of single spore cultures ……………………...........................................54

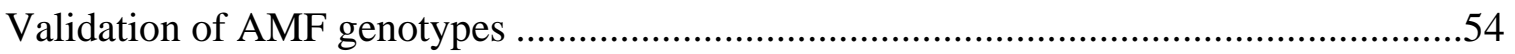

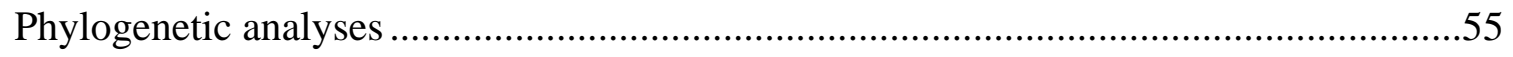

Criteria to establish pairings between isolates ..............................................................5

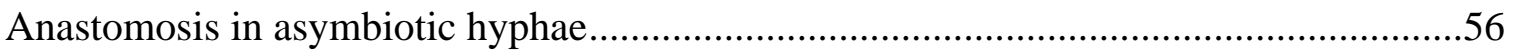

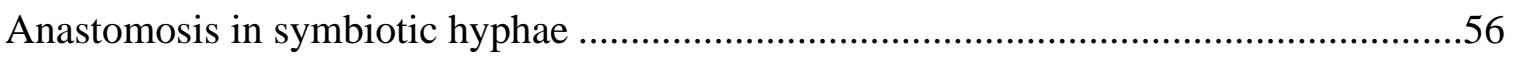

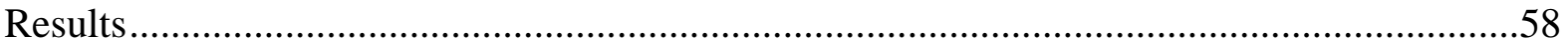

Genetic diversity of Glomus clarum at pasture and mining sites .....................................58

Anastomosis in asymbiotic mycelia...............................................................................

Anastomosis in symbiotic mycelia ………………….................................................59

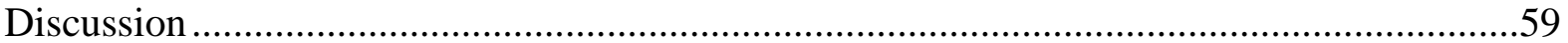

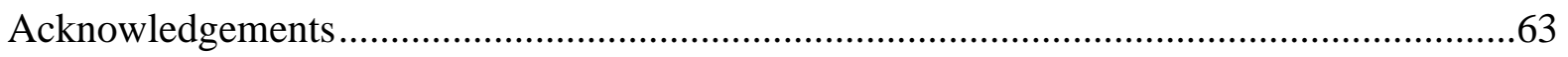

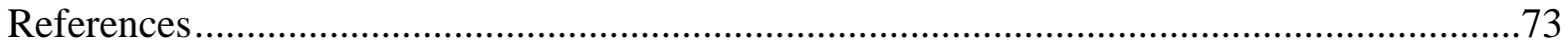

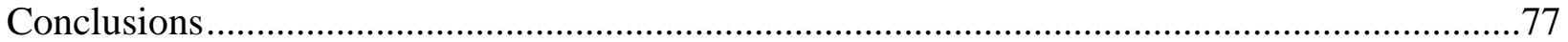

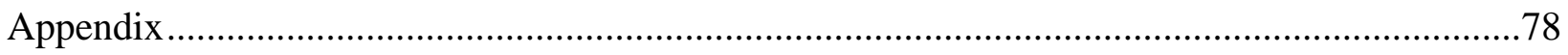

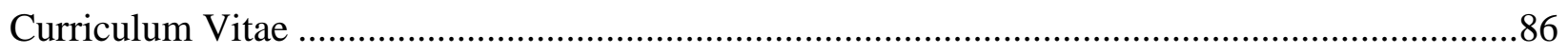




\section{LIST OF TABLES}

Review of literature - Table 1. Number of vegetative incompatibility genes, vegetative compatibility groups, and type of interaction in some species of Ascomycete fungi. Fonts:

Glass \& Kuldau (1992), Glass et al. (2000)

Chapter 1 - Table 1. Accessions obtained from the INVAM for the in situ evaluation of anastomosis

Chapter 1 - Table 2. Type and number of hyphal contacts indicative of compatibility and/or incompatibility of symbiotic external hyphae from the six glomeromycotan species tested, as measured on a $1 \times 1-\mathrm{mm}$ grid overlaying an agar-coated slide

Chapter 1 - Table 3. Total number of hyphal contacts observed in the six glomeromycotan species tested. The hyphal contacts were classified as "compatible," "incompatible," and "no recognition." The frequency of contacts (calculated as a percentage) was determined from the number of hyphal contacts in each category divided by the total number of contacts

Chapter 2 - Table 1. Microsatellite repeats used as PCR primers to amplify flanking regions in genomic DNA extracted from a pooled population of Glomus clarum spores

Chapter 2 - Table 2. Primer pairs used to amplify microsatellite-flanking regions from single spore cultures of Glomus clarum. Primers were designed from ten amplicons generated by microsatellite-primers (Table 1)

Chapter 2 - Table 3. Percentage of hyphal contacts that resulted in compatible hyphal fusion (self anastomosis) in relation to the total number of hyphal contacts observed. Fusions were observed in germinating spores (asymbiotic phase) and in extraradical mycelium of Glomus clarum growing from mycorrhizal plants (symbiotic phase).

Chapter 2 - Table 4. Genetic distance and percentage of contacts that resulted in compatible hyphal fusion (non-self anastomosis) in relation to the total number of hyphal contacts observed. Data from pairings where non-self anastomosis was evaluated in germinating spores (asymbiotic phase) and extraradical mycelium of Glomus clarum growing from mycorrhizal plants (symbiotic phase) 


\section{LIST OF FIGURES}

Review of literature - Figure 1. Events during hyphal fusion (anastomosis) between hyphae of filamentous fungi. (i) initiation, (ii) recognition, (iii) maintenance, (iv) rejection. Adapted from Glass \& Kaneko (2003)

Review of literature - Figure 2. Events during anastomosis between hyphae of Neurospora crassa. a) Homing of hyphal tips. b) Fusion of walls and creation of a fusion pore (arrow). c) Intermixing of cytoplasmic contents. d) Movement of a vacuole between compatible hyphae through a fusion pore. e) Evidence of heterokaryon rejection. Note intense vacuolization (black arrowhead) and hyphal degradation (white arrowhead). Bars $=10 \mu \mathrm{m}$. Images from Saupe (2000), Hickey et al. (2002), and Glass \& Kaneko (2003)

Review of literature - Figure 3. Pairings between vegetative compatible (a) and vegetative incompatible (b) colonies of Cryphonectria parasitica. The dark line in (b) reflects the zone of anastomosis between the two colonies where anastomosis fails and the heterokaryont mycelium is destroyed. Source: personal image by author.

Review of literature - Figure 4. Three types of interactions between vegetative incompatibility genes. For fungal individuals to be compatible, all alleles must be identical (top). If one allele is different, interacting individuals are vegetative incompatible (bottom). Adapted from Glass \& Dementhon (2006)

Review of literature - Figure 5. Four possible vegetative compatibility groups (VCGs) considering 2 vegetative incompatibility genes (het-c and pin-c), each one with two alleles (c1 and $c 2$ ). Source: personal image by author.

Review of literature - Figure 6. Compatible and incompatible hyphal fusion in AMF. a) Long bridge in G. mosseae, anastomosis is evidenced by formazan salt depositions. From Giovannetti et al. (2001), bar $=10 \mu \mathrm{m}$. b) Short bridge in $G$. mosseae, notice fusion of hyphal walls and establishment of protoplasmic continuity. From Giovannetti et al. (2004), bar = 10 $\mu \mathrm{m}$. c) Post-fusion incompatibility in $G$. intraradices; protoplasm withdrawal and septum formation (arrow) in one of the fused hyphae after anastomosis. From Croll et al. (2009). d) Post-fusion incompatible interaction in $G$. mosseae, evidenced by formation of septa separating fused compartments. Reprinted with permission from Mycologia. CThe Mycological Society of America. From Sbrana et al. (2010), bar $=4.5 \mu \mathrm{m}$

Review of literature - Figure 7. Evidence of pre-fusion vegetative incompatibility between isolates of Glomus mosseae. Note swelling, retraction and formation of septa before fusion of hyphae. From Giovannetti et al. (2003). Bars $=35 \mu \mathrm{m}$ in a; $9 \mu \mathrm{m}$ in b

Review of literature - Figure 8. Sorting of genetic variation in AMF according to contrasting (a) heterokaryotic and (b) homokaryotic models. From Pawlowska \& Taylor (2004) 
Chapter 1- Figure 1. Design of a rhizohyphatron used to evaluate interactions among external hyphae of arbuscular mycorrhizal fungi originating from symbiotic mycorrhizal plants. a) Illustration of the assembled unit: a vertical plant compartment containing preinoculated mycorrhizal seedlings, a lateral slide compartment for the placement of two agar-coated glass slides, a nylon mesh with $41-\mu \mathrm{m}$ openings so that only hyphae from the plant compartment are able to grow into the slide compartment, and a nylon mesh-and-cap combination to keep the slides in place and seal the slide compartment. b) An assembled unit containing two 4-week-old mycorrhizal sorghum plants. c) The slide compartment consisting of a $3.4 \times 7-\mathrm{cm}$ PVC tube with a centered $3.3-\mathrm{cm}$ opening to observe and monitor hyphal growth on top slide and plastic supports for glass slides made from pot labels and affixed with $100 \%$ silicon. d) End cap and nylon mesh with $40-\mu$ m openings to cover each end of the pictured slide compartment

Chapter 1- Figure 2. a) Illustration of AMF mycelia growing on a cover slip coated with agar. b) cover slip overlaying a $1 \times 1-\mathrm{mm}$ grid to evaluate anastomosis. The hyphal tip most distal from the side of the cover slip nearest the plant compartment was identified as the reference point for measurements. Hyphal contacts were evaluated in alternate squares of 1mm-wide columns (labeled 1-11) starting at the reference edge

Chapter 1- Figure 3. Hyphal contacts in the two Glomus species that produced compatible hyphal fusions (anastomosis). a) and b) Formation of a short bridge between the hyphae of $G$. intraradices in close proximity. c) Formation of a long bridge between the more distant hyphae of $G$. intraradices. d) Fusion of walls of $G$. clarum hyphae in direct contact with each other. Bars $=25 \mu \mathrm{m}$

Chapter 1- Figure 4. Hyphal contacts suggestive of vegetative incompatibility in two AMF species. a) Septa formation in a hypha of G. clarum. b) Change in shape of a hyphal branch of G. clarum. c) Septation and change in shape of a hyphal branch of G. clarum. d) No recognition between the hyphae of $G$. gigantea . Bars $=25 \mu \mathrm{m}$

Chapter 2- Figure 1. Map of West Virginia state (USA) indicating sampled locations and respective distances. 6AmA, Valley, $1 \mathrm{UnC}$, CRwest, CRcenter and CReast are abandoned coal stripmine sites near Morgantown. WV101 originated from a pasture near Cassville. WV123A and WV310 originated from sites in 4-6 year-old hydroseeded pasture mixture near Logan, WV. Two single spore cultures were obtained from each sampling population (except CReast, with one culture)

Chapter 2- Figure 2. Neighbor-joining tree reconstructed from two concatenated sequences of microsatellite-flanking regions amplified from the G. clarum genome. Numbers at nodes indicate bootstrap values $\geq 50 \%$. The model of nucleotide substitution is $\mathrm{TrN}+\mathrm{I}$, with a rate substitution matrix of $A$ to $G$ at 14.34 and $C$ to $T$ at 6.25 and proportion of invariable sites of 0.97

Chapter 2- Figure 3. Plant-fungal growth system (rhizohyphatron) designed to study anastomosis in isolates of Glomus clarum. a) Illustration of a single plant compartment unit. The cover slip compartment provides placement for three agar-coated cover slips. Nylon 
mesh with $41 \mu \mathrm{m}$ openings (Sefar America Inc., Depew, NY) separates contents of the plant growth compartment from the cover slip compartment. Nylon mesh + cap tightly seal open end of the cover slip compartment. b) Assembled single unit containing sorghum seedlings preinoculated with spores. c) Illustration of a two plant compartment unit. Nylon mesh (blue lines) blocks roots so that only hyphae grow onto agar-coated cover slips in the cover slip compartment. d) Open rhizohyphatron showing mesh barrier, plastic supports for agar-coated cover slips spaced 1-cm apart in the center compartment, and sorghum seedlings preinoculated with spores

Chapter 2- Figure 4. Non-self anastomosis between asymbiotic hyphae of Glomus clarum. a) Fusion between hyphae of isolates WV123A\#6 and WV123A\#7, with insert showing complete wall fusion and protoplasm continuity, bar $=25 \mu \mathrm{m}$. b) Paired spores of the isolates $1 \mathrm{UnC} \# 5$ and $1 \mathrm{UnC} \# 7$, bar $=200 \mu \mathrm{m}$. c) Detail of region marked in (b) by a rectangle, bar $=$ $25 \mu \mathrm{m}$

Chapter 2- Figure 5. Self anastomosis between symbiotic hyphae of Glomus clarum from

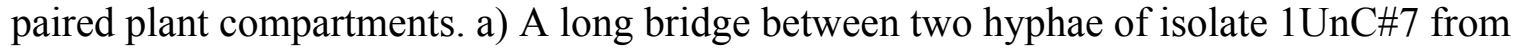
opposite compartments (arrows), bar $=100 \mu \mathrm{m}$. b) Another long bridge from isolate 1UnC\#7 forming between two hyphae from opposite compartments passing each other (arrows). Cytoplasmic flow into the bridge is evident, bar $=50 \mu \mathrm{m}$

Appendix -Figure 1. Germinating spores of Glomus clarum isolates WV123A\#6 (left) and $1 \mathrm{UnC} \# 7$ (right) on a cellophane membrane over M medium. Note hyphal contacts (arrows) in regions where hyphae overlap. Bar $=100 \mu \mathrm{m}$

Appendix -Figure 2. Germinating spores of the Glomus clarum isolates WV123A\#6 on a cellophane membrane over $\mathrm{M}$ medium. The main hypha from each spore fused to form one continuous hypha. Bar $=100 \mu \mathrm{m}$

Appendix - Figure 3. Germinating spores of Glomus clarum isolates 6AmA\#2 (left) and

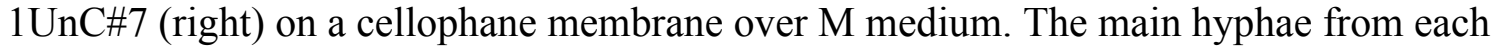
spore are directed away from each other and thereby avoid any physical contact. Bar $=100$ $\mu \mathrm{m}$

Appendix - Figure 4. Germinating spore of Glomus clarum isolate WV123A\#7 on a cellophane membrane over M medium. The main hypha branches immediately after emergence from the broken end of the subtending hypha. Two "spore-like" structures (arrows) are formed 14 days after germination. Bar $=25 \mu \mathrm{m}$

Appendix - Figure 5. Symbiotic mycelium of Glomus clarum isolate CRwest\#8 growing on a cover slip coated with $1.5 \%$ agar. Bar $=100 \mu \mathrm{m}$

Appendix - Figure 6. Symbiotic mycelia of Glomus clarum isolates 1UnC\#7 (left) 6AmA\#3 (right) growing on a cover slip coated with 1.5\% agar. Hyphae from each isolate originated from mycorrhizal plant compartments in direct contact with the cover slip. Bar $=100 \mu \mathrm{m}$ 
Appendix - Figure 7. Self anastomosis in symbiotic mycelium of Glomus clarum isolate 1 UnC\#7 growing on a cover slip coated with $1.5 \%$ agar. Two long bridges are formed (arrows) together with one spore. Bar $=50 \mu \mathrm{m}$

Appendix - Figure 8. Symbiotic mycelium of Glomus clarum isolate 6AmA\#2 growing on a cover slip coated with $1.5 \%$ agar. Intense sporulation occurs 6 weeks after inoculation of sorghum seedlings. Bar $=200 \mu \mathrm{m}$ 


\section{REVIEW OF LITERATURE}

\section{Arbuscular mycorrhizal fungi}

Arbuscular mycorrhizal fungi (AMF, Glomeromycota) are obligate symbionts that colonize roots of most land plant species (Wang \& Qiu, 2006). AMF are unique among fungi in that their life cycle can be divided into discrete asymbiotic and symbiotic phases. Spores in soil begin the life cycle because they are dispersed as individual propagules and then germinate to produce a new autonomous individual (Smith \& Read, 2008). In the absence of a host plant, survival of a germinating spore rarely exceeds 20-30 days (Bonfante \& Perotto, 1995). The hyphae branching from a spore germ tube characterizes the asymbiotic phase, during which the fungus grows to seek a root and establish an obligate mycorrhizal association with a plant.

When an asymbiotic hypha establishes contact with the surface of a root, an appressorium forms on the root epidermis. Infection hyphae are produced from the appressorium and penetrate into the root cortex (Garriock et al., 1989). The establishment of mycorrhizal colonization characterizes the beginning of the dominant symbiotic phase, which may last for as long as the plant host lives. AMF form specialized structures associated with intraradical hyphae that are unique to the mycorrhizal association: intracellular branching arbuscules or terminal intercellular vesicles (Gianinazzi, 1991). Arbuscules are formed between the plant cell wall and membrane, and mediate nutrient exchange between plant and fungus (Harrison, 1997).

Extraradical hyphae grow from roots 2-4 weeks after establishment of colonization (Jakobsen et al., 1992). These hyphae spread through soil aggregates and absorb nutrients (mainly phosphorus) that are transferred to the plant symbiont through the arbuscules (Harrison, 1999). Spores are produced either isolated or in aggregates from different parts of vegetative hyphae in soil or roots within weeks to months of mycorrhizal development (Smith \& Read, 2008). Throughout the entire fungal life cycle, no regular partitioning of hyphae is observed nor are any specialized structures produced to control migration of multiple nuclei through the fungal thallus or to spores (Jany \& Pawlowska, 2010). As a mycelium grows and expands, hyphae that establish physical contact with each other may undergo fusion (anastomosis). This phenomenon is poorly understood in AMF, but it has been well described in Ascomycetes. 


\section{Hyphal fusion in Ascomycetes}

Of all fungal groups, anastomosis is best understood in Ascomycetes. Phenotypic events associated with anastomosis are similar across species. Events include (i) initiation, (ii) recognition and either (iii) maintenance of the heterokaryon or (iv) rejection of the heterokaryon (Fig. 1).

During initiation, hyphal tips or pegs grow towards each other (Fig. 1i, 2a) and establish physical contact. Parts of the walls from both hyphae are broken down by enzymes. A fusion pore then is formed, through which protoplasmic continuity is established (Fig. 1ii, 2b, c). All steps during initiation occur independent of the genetic identity of vegetative incompatibility loci in either of the interacting hyphae.

During recognition, protoplasmic contents from both fungi are intermixed in a distinct zone that becomes a heterokaryon (Fig. 1ii). This condition is defined as heterokaryosis because interacting fungi likely differ at one or more loci in the genome. The newly formed heterokaryont sector may or may not be stable, depending on the genetic identity of recognition genes from both hyphae. Several genes determining stability of the heterokaryon are identified as vegetative incompatibility (vic), heterokaryon incompatibility (het) or partner incompatibility (pin). Proteins produced by vic, het and pin loci interact and form dimers. If all alleles are identical between anastomosing hyphae, homodimers are formed and the heterokaryon is stable. If only one allele is different at these loci, a heterodimer is formed that triggers destruction of the heterokaryont mycelium (Glass \& Kuldau, 1992; Glass \& Dementhon, 2006).

For maintenance of the heterokaryon, alleles of all vegetative incompatibility loci must be identical (Fig. 1iii, 2d). After fusion, the apical compartment undergoes cell division and a new heterokaryont mycelium sector is created (Newhouse \& MacDonald, 1991; McCabe et al., 1999). The result is vegetative (or somatic) compatibility. The heterokaryon is stable until nuclear segregation eventually occurs through asexual sporulation or hyphal branching and restores homokaryosis.

Rejection of the heterokaryon occurs when hyphae that differ genetically at any incompatibility loci fuse and then are killed (Fig. 1iv, 2e). This process is indicative of vegetative (or somatic) incompatibility. Degradation of the heterokaryont mycelium occurs by programmed cell death (PCD). PCD during anastomosis occurs by autophagy (Marek et al., 2003; Paoletti \& Clave, 2007), which contrasts with apoptosis, a form of PCD in animal cells (Pinan-Lucarré et 
al., 2003). In apoptosis, cells shrink and fragment but are not completely degraded. Organelles that are not harmful can be phagocytosed and recycled by the organism. With autophagy, however, cell contents are totally destroyed and cellular compartments are not recycled (reviewed by Bursch, 2001).

The first step of PCD in heterokaryons is the plugging of septal pores by proteins, which compartmentalizes the hyphal region undergoing an incompatible reaction. Autophagosomes engulph protoplasmic components and target them for degradation in vacuoles (Fig. 2e).

Proteases and laccases are produced to degrade fungal cell walls and proteins (Boucherie \& Bernet, 1978; Paoletti et al., 2001). Three of these enzymes have been genetically characterized. Because of expression only during autophagy, these genes are classified as idi (induced $d$ uring incompatibility) genes (Saupe, 2000). Progressive loss of cytoplasmic density is observed during autophagy until the entire heterokaryon is degraded, followed by mycelium death and cell lysis (Marek et al., 2003). The zone between two incompatible colonies is manifested phenotypically as a dark line devoid of mycelium (Fig. 3).

Vegetative incompatibility in Ascomycete fungi is regulated by two genetic systems, one allelic and the other non-allelic. In the allelic system, proteins produced by the same locus interact to form dimers. In non-allelic systems, interaction occurs between products of alleles from two or more closely-linked or unlinked loci (Fig. 4). Regardless of the type of interaction, all alleles must be identical for fungi to be vegetative compatible.

The number of genes involved in vegetative incompatibility and the type of allelic interaction varies with fungal species (Table 1). Based on the number of genes and alleles, it is possible to estimate the number of vegetative compatibility groups (VCGs) in fungal populations. Every unique allelic combination constitutes a VCG (Fig. 5).

Vegetative incompatibility genes are highly polymorphic, increasing the number of possible VCGs in fungal populations. When polymorphic, all incompatibility genes but one (het$s$ ) have a HET domain, suggesting that this specific domain is involved in dimer formation during heterokaryon formation (Paoletti \& Saupe, 2009). Although several species have been reported to possess vegetative incompatibility systems, genes have been cloned and characterized genetically only in Podospora anserina and Neurospora crassa. 


\section{Incompatibility systems in Podospora anserina}

At least 17 loci are involved in both allelic and non-allelic heterokaryon incompatibility in $P$. anserina (Glass et al., 2000). Of those interactions, the het-s locus is unique in that the HET domain is absent.

The het-s locus can exist as either het-s and het-S alleles. The het-s allele expresses two phenotypes: prion-free [Het-s*] and prion-infected [Het-s]. Prion-free fungi acquire prions from infected individuals upon physical contact. Fungi expressing the het-S allele are prion-free, with the phenotype $[$ Het-S]. When undergoing hyphal fusion, [Het-s*] and [Het-S] individuals are able to anastomose. Vegetative incompatibility and cell death occur only when hyphae of a [Hets] phenotype fuses with that of the [Het-S] phenotype. The prion in [Het-s] induces misfolding of various proteins and triggers programmed cell death (Saupe, 2007).

The best described non-allelic system comprises three closely-linked loci: het-c, het-d and het-e. Four het-c, four het-e, and three het-d alleles have been described. Each allele in het-c is incompatible with a specific allele of het- $d$ and het-e alleles, respectively (Saupe, 2000; Glass $\&$ Dementhon, 2006). The gene het-c encodes for a glycolipid transfer protein (Saupe et al., 1994). The genes het- $d$ and het-e encode signal transduction ATPases with numerous domains (STAND proteins). These proteins have three domains: a $\mathrm{N}$ terminal HET domain, a central NACHT domain and a C terminal WD-repeat domain. (Saupe et al., 1995; Paoletti et al., 2007). HET-C proteins also have cellular functions other than vegetative incompatibility. These proteins appear to be incorporated into the hyphal cell wall, so that formation of heterodimers in heterokaryon incompatibility interferes with wall synthesis and contributes to cell death (Saupe et al., 1994).

The loci het-r and het-v are not linked and interact via a non-allelic mechanism. The het$r$, het- $d$ and het-e loci all encode STAND proteins (Chevanne et al., 2009). The het- $v$ has yet to be cloned and characterized.

\section{Incompatibility systems in Neurospora crassa}

At least 11 vegetative incompatibility genes have been identified in $N$. crassa, including one mating type gene (mat). All of the characterized heterokaryon incompatibility reactions appear to function through an allelic mechanism, with the exception of mat. 
The het-C locus has three allelic forms: het $C^{\mathrm{OR}}$, het $-C^{\mathrm{PA}}$ and het $-C^{\mathrm{GR}}$. These alleles encode glycine-rich proteins associated with the fungal cell walls. Heterodimer formation therefore affects normal growth and morphology (Saupe et al., 1996; Saupe \& Glass, 1997).

The locus het- 6 has two alleles: het $6^{\mathrm{PA}}$ and het $-6^{\mathrm{OR}}$. The similarity between those two alleles is only $68 \%$, the lowest level reported between alleles of a vegetative incompatibility locus. het $-6^{\mathrm{PA}}$ and het $-6^{\mathrm{OR}}$ mediate heterokaryon destruction through a non-allelic interaction with un-24 alleles (Smith et al., 2000). However, how the interaction triggers cell death is not clear.

The locus $u n-24$ has two characterized alleles: $u n-24^{\mathrm{OR}}$ and $u n-24^{\mathrm{PA}}$. In other fungi, $u n-24$ encodes the large subunit of ribonucleotide reductase. Interestingly, this enzyme in N. crassa has a unique carboxy-terminal domain that confers the heterokaryon incompatibility function (Smith et al., 2000).

Two alleles are identified in the het-I locus: het-I and het-i (Pittenger \& Brawner, 1961). Interaction of these alleles does not produce a full heterokaryotic mycelium sector. Instead, heterokaryons are lost by the destruction of only one nuclear component.

The mat gene is the only known example of non-allelic interactions in N. crassa. This gene is also involved in sexual incompatibility, conferring different mating types: $A$ and $a$. Mating type $A$ has three possible idiomorphs: mat $A-1$, mat $A-2$ and mat $A-3$. Mating type $a$ has only one idiomorph, mat $a-1$. All idiomorphs are involved in sexual incompatibility, but only mat $A-1$ and mat a-1 are involved in vegetative incompatibility. During mating, all alleles must be different. Successful vegetative interactions, however, require that all alleles are identical. Vegetative incompatibility is silenced during heterokaryon formation through proteins produced by an unlinked locus, tol. Transcription of tol makes the different nuclei tolerant to genetic differences $($ tol $=$ tolerant $)$. This is the only molecularly characterized mediator of allelic incompatibility in fungi (Shiu \& Glass, 1999).

\section{Occurrence and significance of anastomosis in Ascomycetes}

Anastomosis occurs frequently between hyphae of a same colony and also between different colonies. A fungal colony produced by a single spore is considered a ramet, which is loosely equivalent to an individual (Buss, 1983). Therefore, hyphal fusion within a colony is considered "self anastomosis". Fusion between hyphae produced by two separate colonies, or 
"individuals", is considered "non-self anastomosis" (Glass \& Kaneko, 2003; Glass et al., 2004). Non-self anastomosis can occur between both genetic similar or genetic different colonies.

Several hypotheses have been proposed to explain the occurrence of vegetative compatibility. Aanen et al. (2008) examined costs and benefits of vegetative compatibility, and they proposed hypotheses that seem consistent with patterns observed for most fungal species studied to date, especially those in the Ascomycetes. They suggest that self anastomosis is beneficial and selects for compatibility. Self compatibility within a mycelium is important for redistribution of water, nutrients and cytoplasmic contents (Gregory, 1984). On the other hand, non-self anastomosis is costly and therefore selects for incompatibility. Non-self anastomosis might represent fitness loss for one of the partners because of frequent nuclear parasitism (Debets \& Griffiths, 1998) and transmission of viruses or other harmful cytoplasmic elements (Biella et al., 2002). However, non-self anastomosis is not always costly. Exchange of nuclei between fungi is important to create genetic variability by mitotic recombination in the heterokaryont condition. This event might not be critical for fungi that alternate sexual and asexual stages, because heterokaryons are formed in the sexual stage and meiotic recombination generates variability (Coppin et al., 1997). Yet vegetative non-self compatibility might be very important for exclusively asexual fungi such as those in Glomeromycetes. In the absence of sexual reproduction, anastomosis between individuals would provide the only means for gene flow between populations.

\section{Vegetative compatibility in AMF}

Anastomosis has been studied mostly in the asymbiotic phase of the AMF life cycle, which consists of all events and processes from spore germination to contact with a host plant. Studies on anastomosis during the symbiotic phase, or events and processes after establishment of a mycorrhizal association, are rare because of methodological constraints. Mycelium behavior in colonized plants (Giovannetti et al., 2001; 2004) is difficult to observe and measure because hyphae are very sensitive to damage when manipulated. In vitro root organ cultures (Bécard \& Fortin, 1988) are useful for observation of hyphal fusions, but a majority of AMF species cannot be successfully cultivated in vitro. In this dissertation, a new cultivation system (rhizohyphatron) was invented to allow evaluation of anastomosis in symbiotic mycelia without constrains that are typically observed in other systems. The rhizohyphatron has two variations; one designed to 
evaluate self anastomosis in mixed populations of six AMF species (Chapter 1) and the other designed to evaluate both self and non-self anastomosis of defined genotypes in the species Glomus clarum (Chapter 2).

The definition of "self" or an AMF "individual" has been subjected to intense and endless debate. Some researchers have considered all spores from one accession to represent a ramet. All hyphal fusions observed within a mycelial network originating from a sampled population were described as self anastomosis (Giovannetti et al., 2003). However, this definition is questionable because one sample might contain two or more different genotypes so that the term "self" is not valid. More recently, the term "self" has been applied only to a single spore or a culture derived from a single spore inoculum (same ramet). The term "non-self" is used to denote interactions between different spores or between different single spore derived cultures (Croll et al., 2009). These will be the definitions adopted throughout this dissertation. When multiple spores are used as an inoculum source to study anastomosis, the interactions observed will be described as a "population" rather than an "individual".

\section{Vegetative compatibility within populations}

The first study of vegetative compatibility in AMF was documented by Tommerup (1988). She reported the occurrence of anastomosis between isolates of Acaulospora laevis, Archaeospora trappei, Glomus caledonium, G. monosporum, G. fasciculatum, G. tenue, Gigaspora decipiens and Scutellospora calospora. Because data concerning origin of these isolates, frequency of anastomosis, and experimental growth conditions were not documented, interpretation of results was of limited value. After 15 years, other studies were carried out, but inocula still consisted of multiple spores.

Evidence for hyphal fusion in the asymbiotic phase has been found only in Glomus species (Giovannetti et al., 1999; 2003; Cardenas Flores et al., 2010). In this group, the frequency of anastomosis ranges between 40-90\%. No anastomosis has been found in the mycelia of Scutellospora and Gigaspora species (Giovannetti et al., 1999), and it has not been studied in genera such as Paraglomus and Ambispora.

Anastomosis in symbiotic mycelium has been studied only in Glomus. In root organ cultures, $100 \%$ of hyphal contacts in mycelia of G. proliferum, G. intraradices and G. hoi had resulted in fusions (de la Providencia et al., 2005). In whole plant associations with G. mosseae, 
the frequency of anastomosis is considerably lower (36-78\%) (Giovannetti et al., 2001; 2004). Anastomosis frequency was similar within the mycelium of a single plant or between mycelia of paired plants.

Anastomosis has been studied in populations of only three out of 15 AMF genera. Two main constraints have limited advancements in research on anastomosis in symbiotic mycelia. The first is reliance on cultivation systems which do not reflect whole plant-fungus interactions. In vitro root organ cultures constitute atypical growth conditions, where the plant host consists of immortalized root explants subjected to high nutrient levels (de la Providencia et al., 2005; Voets et al., 2006). The second is the low number of species capable of being cultured in vitro. The main reason little is known about anastomosis in groups other than Glomus is their lack of compatibility with the root organ culture environment.

\section{Self and non-self anastomosis}

Self anastomosis in the asymbiotic phase has been studied only in $G$. intraradices. When germinating spores were paired, 46-51\% of the hyphal contacts led to fusion (Croll et al., 2009). Self anastomosis in symbiotic mycelium has been studied in a few species of Glomeraceae and Gigasporaceae using the in vitro culture system. In Glomus proliferum and G. intraradices, percentage of anastomosis in hyphae produced by a mycorrhizal root organ was of 80 and $88 \%$, respectively (Voets et al., 2006). In Gigaspora and Scutellospora, the frequency of hyphal fusion is less than 10\% (de Souza \& Declerck, 2003; de la Providencia et al., 2005; Voets et al., 2006). When fusion is evaluated between hyphae from two mycorrhizal root organs, the percentage of anastomosis decreases dramatically in Glomus and is completely absent in Gigaspora and Scutellospora (Voets et al., 2006). These observations suggest that recognition phenomena differ in a single mycelium versus paired mycelia of an AMF.

Non-self anastomosis has been studied only in germinating spores of defined genotypes of G. intraradices (Croll et al., 2009). Frequency of non-self anastomosis ranged between 0 and $10 \%$, and some concomitant variation in phenotypes of mycorrhizal plants suggests that lateral gene transfer occurred. Information on non-self anastomosis in the symbiotic phase has yet to be obtained on any AMF species, even though this phase is lengthy and crucial to success of the symbiosis. 


\section{Morphology of hyphal interactions}

When two colonies of Ascomycete fungi are paired, anastomosis events either lead to successful fusion or destruction of the heterokaryon. When colonies meet, there is a typical tipto-tip fusion (Fig. 1) and if individuals are incompatible a zone of hyphal destruction is formed (Fig. 3). Therefore, anastomosis in Ascomycetes is usually scored as either present or absent. When AMF mycelia are paired, no typical tip-to-tip fusion occurs, so hyphae often overlap. Hyphal contacts occur in both the center and periphery of colonies, and any response is not absolute. Some contacts may be incompatible while others result in fusion. Therefore, anastomosis cannot be scored using the same methods used for Ascomycete fungi. Instead, every hyphal contact must be evaluated under a microscope.

Compatible hyphal fusions in AMF mycelium are mainly characterized by the formation of short and long bridges (Giovannetti et al. 2001; 2004; Fig. 6a, b). While vegetative incompatibility is regulated after hyphal fusion in Ascomycetes (Fig. 1, 2), it seems to be evident both before and after fusion in AMF. Post-fusion incompatibility is identified when, following anastomosis, the protoplasm of one hypha withdraws and a septum formed between the two hyphae (Fig. 6c, d). Low levels of post-fusion incompatibility have been observed only in germinating spores of G. intraradices and G. mosseae (Croll et al., 2009; Sbrana et al., 2010). However, no post-fusion incompatibility has been observed when the fungus is in a symbiosis with its host (Giovannetti et al., 2001; 2004; de la Providencia et al., 2005; Voets et al., 2006; Sbrana et al., 2010). Vegetative incompatibility in AMF also is regulated the pre-fusion stage. Before or during physical contact between hyphae, germ tubes swell, branch and form septa (Fig. 7). Physical changes in germ tubes prior to any contact suggest that specific recognition signals are involved during this stage.

\section{Significance of anastomosis in arbuscular mycorrhizal fungi}

Anastomosis is functionally important for AMF for underground resource allocation as well as for healing of wounded or broken hyphae. Fusion between mycelia of Glomus mosseae isolates produces common mycorrhizal networks (CMNs) that allow nutrient transfer between plants (Mikkelsen et al., 2008). So far, the formation of CMNs has been reported only in $G$. mosseae, so the universality of this phenomenon remains unknown. Anastomosis behavior in other AMF species and phylogenetically diverse groups would contribute to a better 
understanding of the genetics of population and community-level interactions. After hyphal damage, new lateral or apical tips are formed and fuse to re-establish cytoplasmic flow (de la Providencia et al., 2005). Hyphal fusion is involved in healing processes in three genera studied so far (Glomus, Scutellospora and Gigaspora).

Knowledge of anastomosis also has implications at the genetic level. The genetic organization of AMF is unclear from available evidence, and two alternative hypotheses have been proposed, one involving homokaryotic and the other heterokaryotic organization of the AMF genome (Fig. 8). Anastomosis would not be important for maintenance of homokaryosis (Pawlowska \& Taylor, 2004), but it would be required to compensate genetic drift created by bottlenecks if heterokaryosis is the dominant process (Bever \& Wang, 2005).

Non-self anastomosis represents the only possibility for gene flow among individuals of asexual fungi. Non-self anastomosis between genetically different germinating spores of $G$. intraradices has been measured at low levels (Croll et al., 2009). However, non-self fusion between genetically different individuals cannot be generalized across AMF lineages for two reasons. First, data so far is confined to results from the study of only one species, $G$. intraradices. Second, no information is available on non-self fusion in symbiotic mycelia. Broader understanding of non-self anastomosis in a range of AMF species and in different stages of the AMF life cycle will help to understand mechanisms of inter-individual interactions.

The present dissertation is divided in two chapters. The objective of the study presented in the first chapter was to assess vegetative compatibility in symbiotic hyphae of populations of species from representative AMF genera. The hypothesis to be tested was that vegetative compatibility is observed in all taxa. The objective of the second chapter was to evaluate self and non-self anastomosis in germinating spores and symbiotic hyphae of Glomus clarum. This study was conducted to test the hypothesis that anastomosis is observed in all phases of the AMF life cycle. 
Table 1. Number of vegetative incompatibility genes, vegetative compatibility groups, and type of interaction in some species of Ascomycete fungi. Fonts: Glass \& Kuldau (1992), Glass et al. (2000).

\begin{tabular}{lccc}
\hline \multicolumn{1}{c}{ Species } & $\begin{array}{c}\text { Vegetative } \\
\text { incompatibility } \\
\text { genes }\end{array}$ & $\begin{array}{c}\text { Vegetative } \\
\text { compatibility } \\
\text { groups* }\end{array}$ & $\begin{array}{c}\text { Type of interaction } \\
\text { Cryphonectria parasitica } \\
\text { Aspergillus nidulans }\end{array}$ \\
$\begin{array}{l}\text { Neurospora crassa } \\
\text { Podospora anserina }\end{array}$ & 11 & 128 & Allelic \\
\hline * calculated on the assumption that each gene has two alleles. Therefore, a species with seven \\
genes and two allelic forms of each gene has 128 vegetative compatible groups $\left(2^{7}\right)$.
\end{tabular}


Individual A

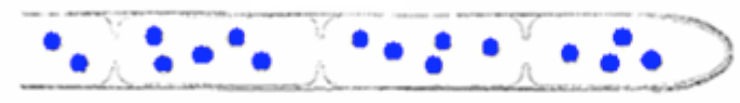

(ii)

(i)

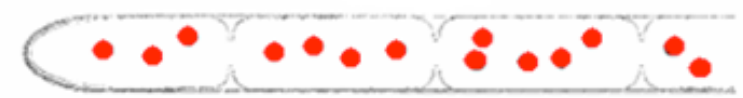

i) $\mathbf{1}$

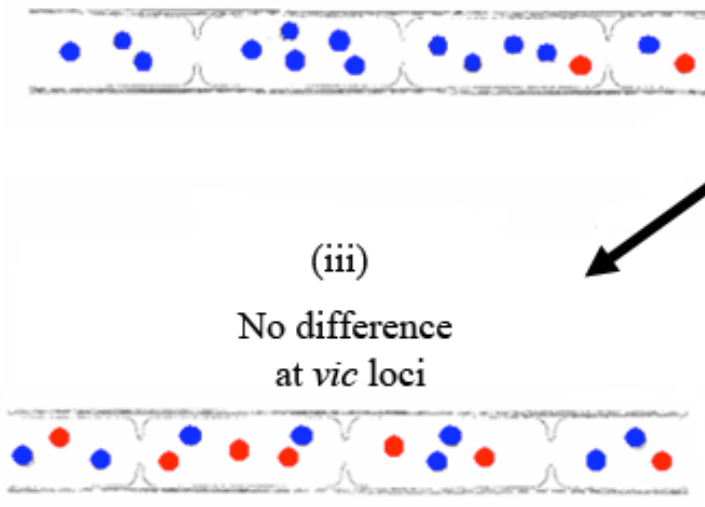

Establishment of a

compatible heterokaryon

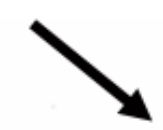

(iv)

Genetic difference at vic locus

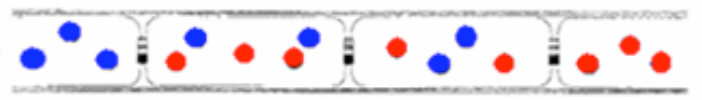

Septal plugging

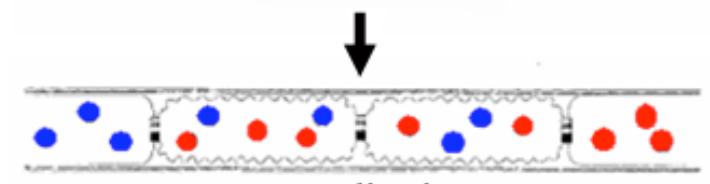

Vacuolization

Shrinkage of plasma membrane

Organelle degradation

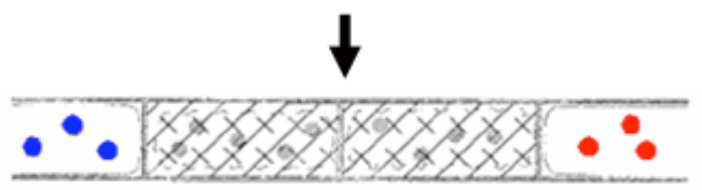

DNA fragmentation

Hyphal compartmentation

Cell death

Figure 1. Events during hyphal fusion (anastomosis) between hyphae of filamentous fungi. (i) initiation, (ii) recognition, (iii) maintenance, (iv) rejection. Adapted from Glass \& Kaneko (2003). 

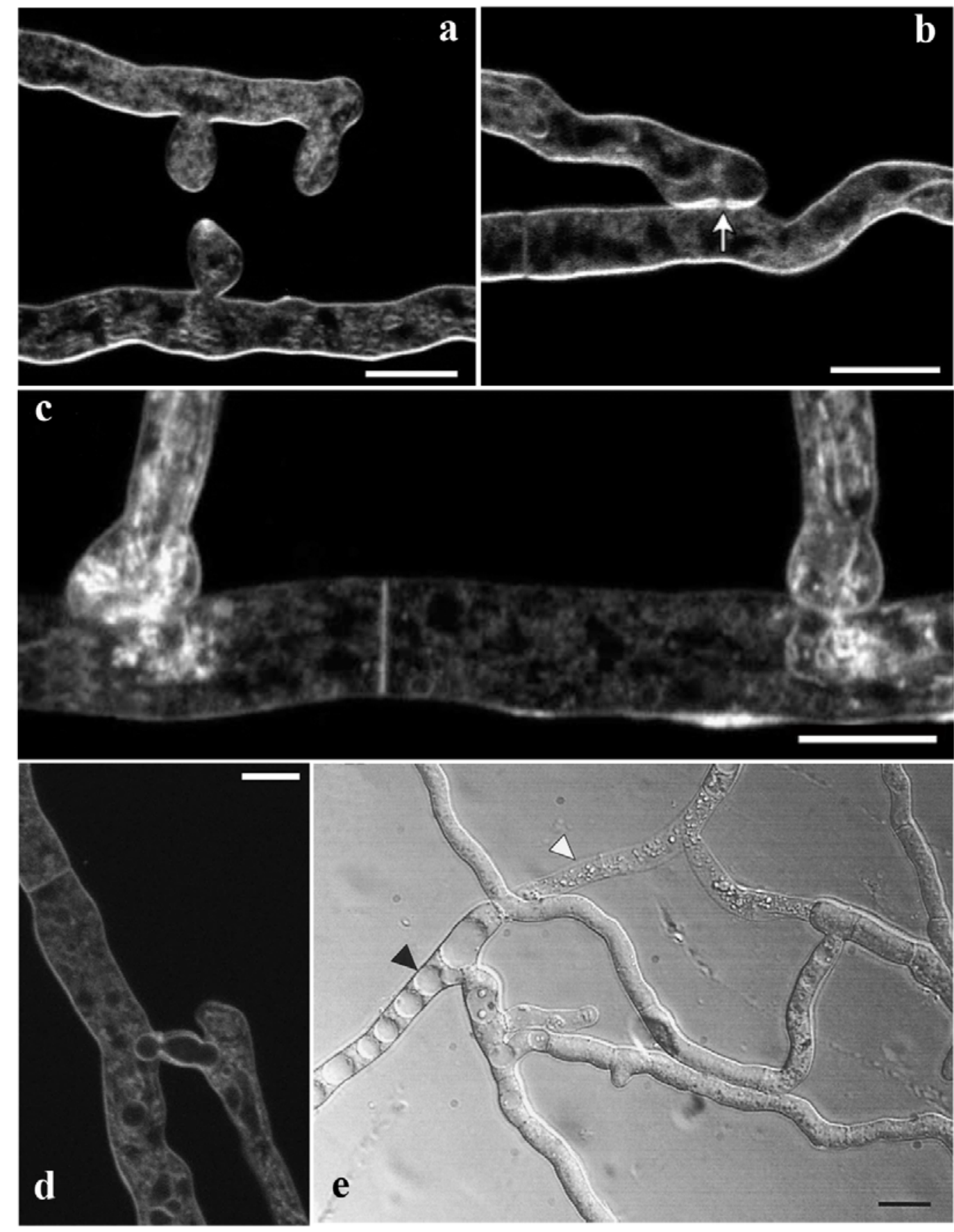

Figure 2. Events during anastomosis between hyphae of Neurospora crassa. a) Homing of hyphal tips. b) Fusion of walls and creation of a fusion pore (arrow). c) Intermixing of cytoplasmic contents. d) Movement of a vacuole between compatible hyphae through a fusion pore. e) Evidence of heterokaryon rejection. Note intense vacuolization (black arrowhead) and hyphal degradation (white arrowhead). Bars $=10 \mu \mathrm{m}$. Images from Saupe (2000), Hickey et al. (2002), and Glass \& Kaneko (2003). 


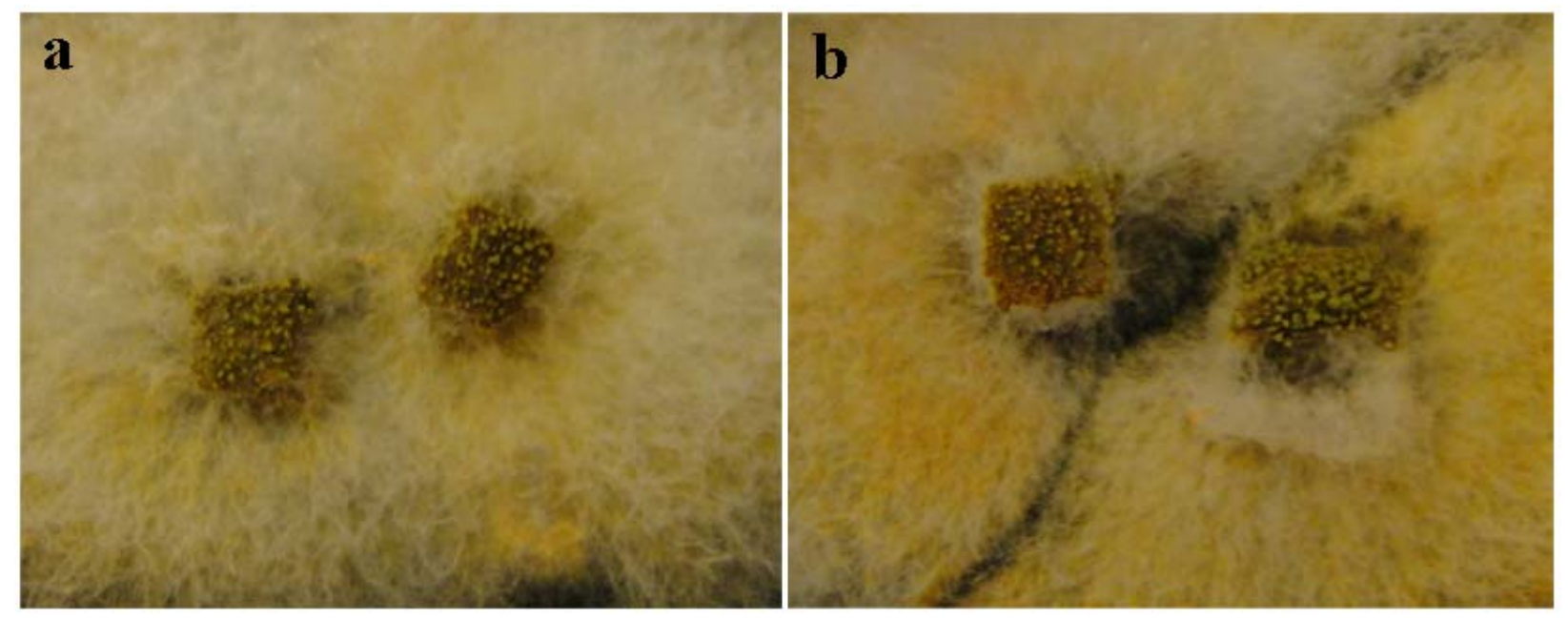

Figure 3. Pairings between vegetative compatible (a) and vegetative incompatible (b) colonies of Cryphonectria parasitica. The dark line in (b) reflects the zone of anastomosis between the two colonies where anastomosis fails and the heterokaryont mycelium is destroyed. Source: personal image by author. 


\section{Vegetative compatible fungi: identity at all alleles}
Allelic Non-allelic interactions: Non-allelic interactions:
interactions closely-linkedgenes unlinkedgenes

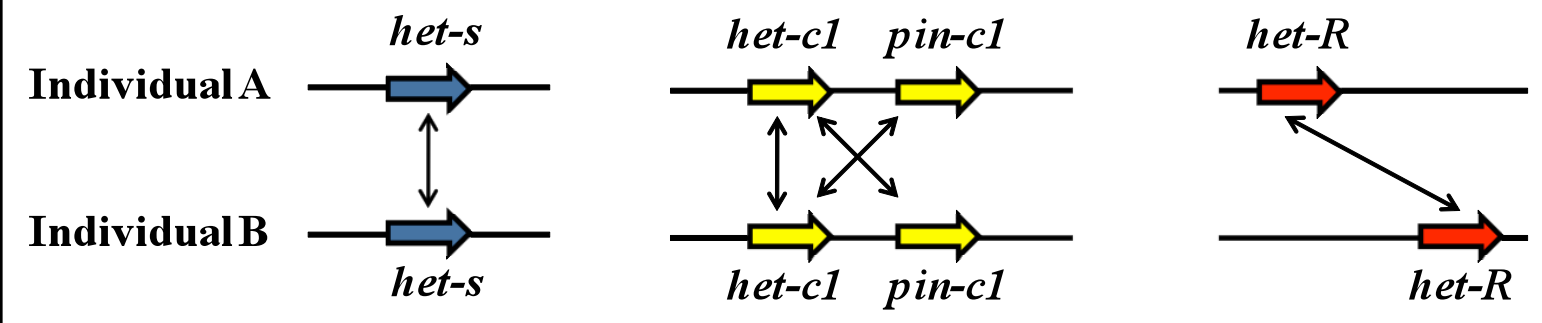

Vegetative incompatible fungi: allelic differences
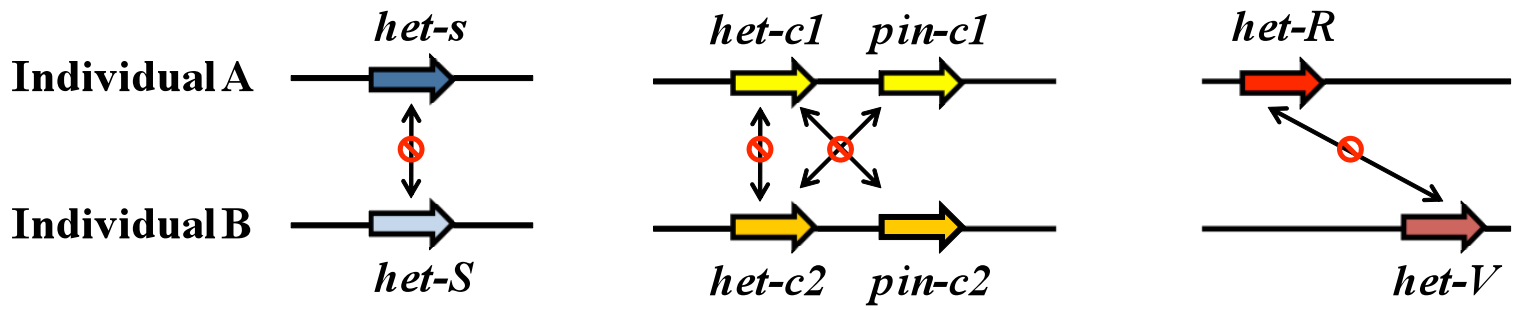

Figure 4. Three types of interactions between vegetative incompatibility genes. For fungal individuals to be compatible, all alleles must be identical (top). If one allele is different, interacting individuals are vegetative incompatible (bottom). Adapted from Glass \& Dementhon (2006). 


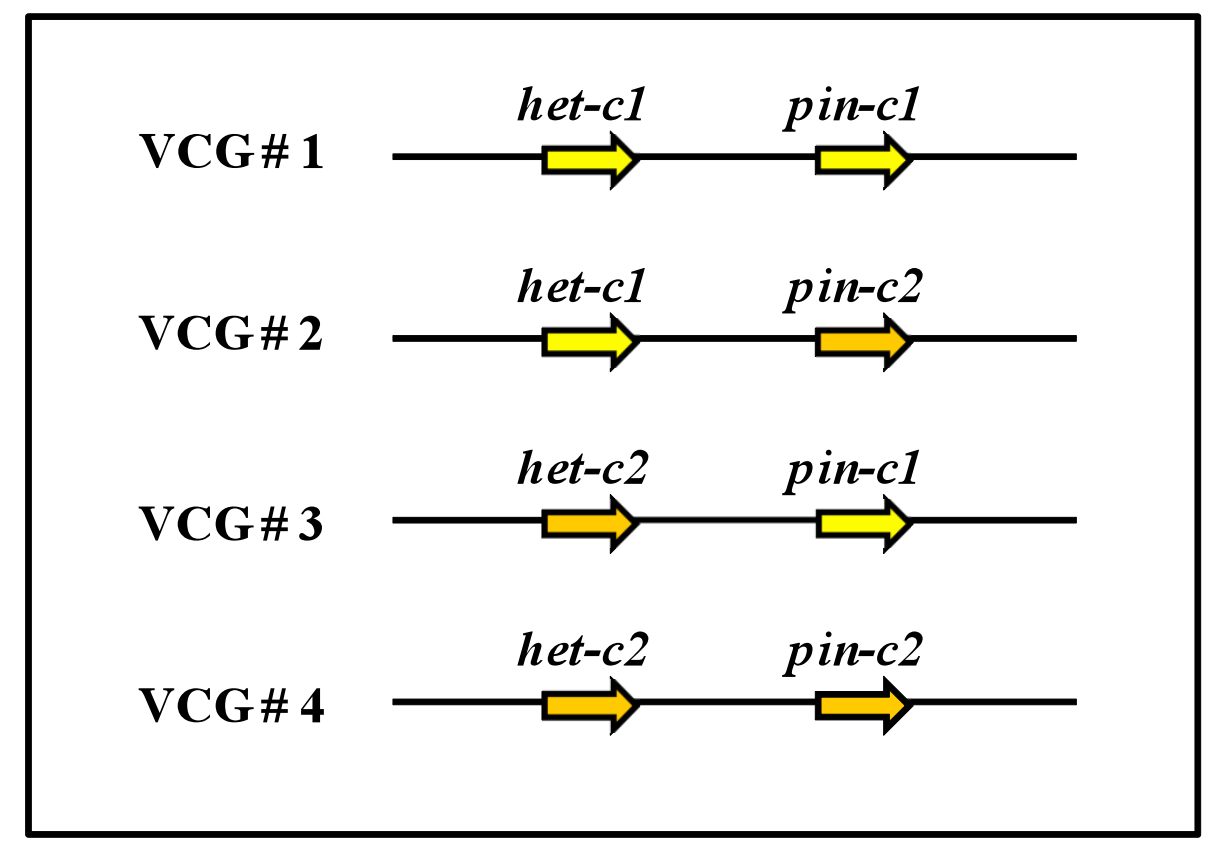

Figure 5. Four possible vegetative compatibility groups (VCGs) considering 2 vegetative incompatibility genes (het-c and pin-c), each one with two alleles ( $c 1$ and $c 2$ ). Source: personal image by author. 


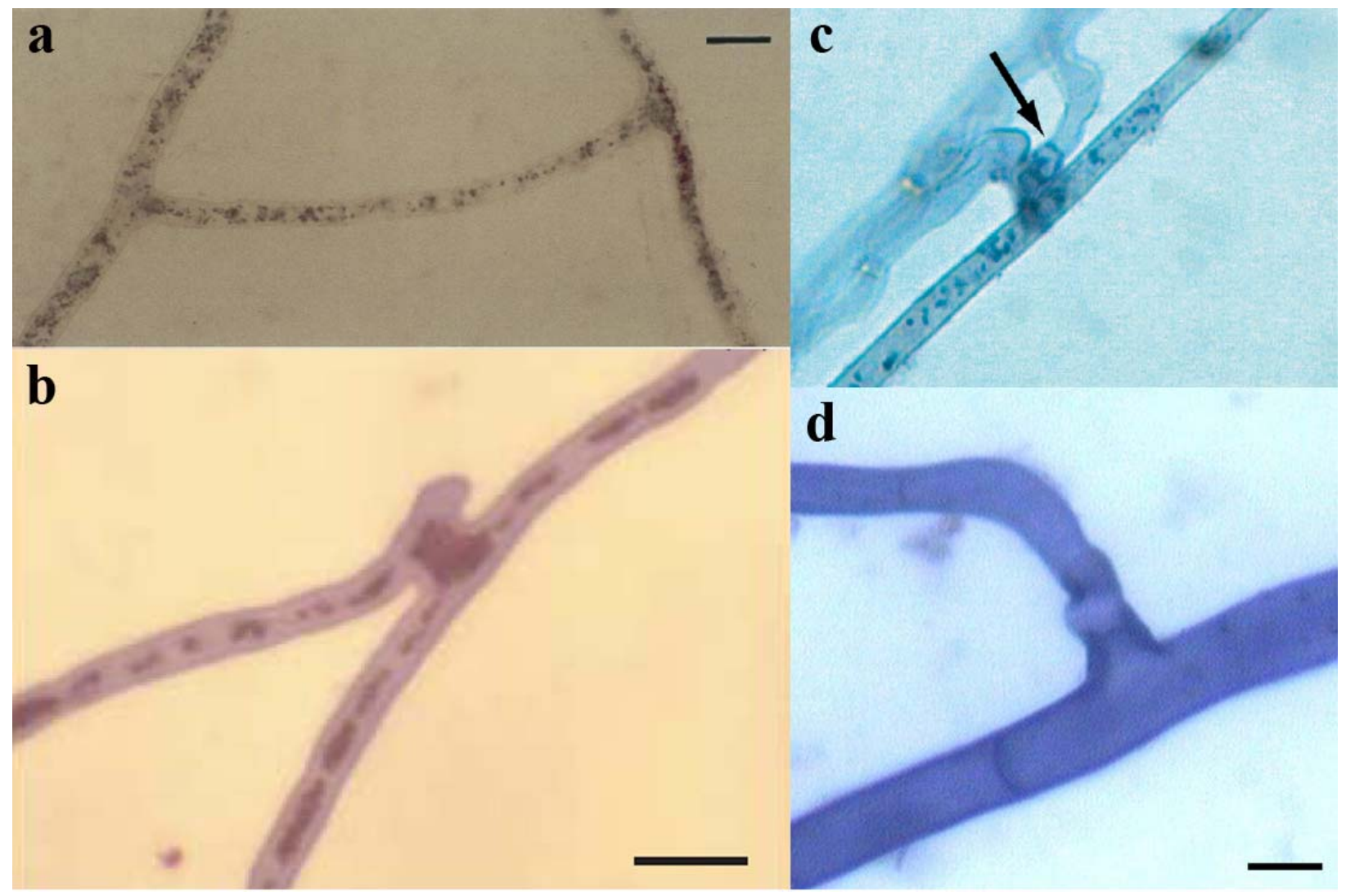

Figure 6. Compatible and incompatible hyphal fusion in AMF. a) Long bridge in G. mosseae, anastomosis is evidenced by formazan salt depositions. From Giovannetti et al. (2001), bar = 10 $\mu \mathrm{m}$. b) Short bridge in G. mosseae, notice fusion of hyphal walls and establishment of protoplasmic continuity. From Giovannetti et al. (2004), bar $=10 \mu \mathrm{m}$. c) Post-fusion incompatibility in $G$. intraradices; protoplasm withdrawal and septum formation (arrow) in one of the fused hyphae after anastomosis. From Croll et al. (2009). d) Post-fusion incompatible interaction in G. mosseae, evidenced by formation of septa separating fused compartments. Reprinted with permission from Mycologia. CThe Mycological Society of America. From Sbrana et al. (2010), bar $=4.5 \mu \mathrm{m}$. 

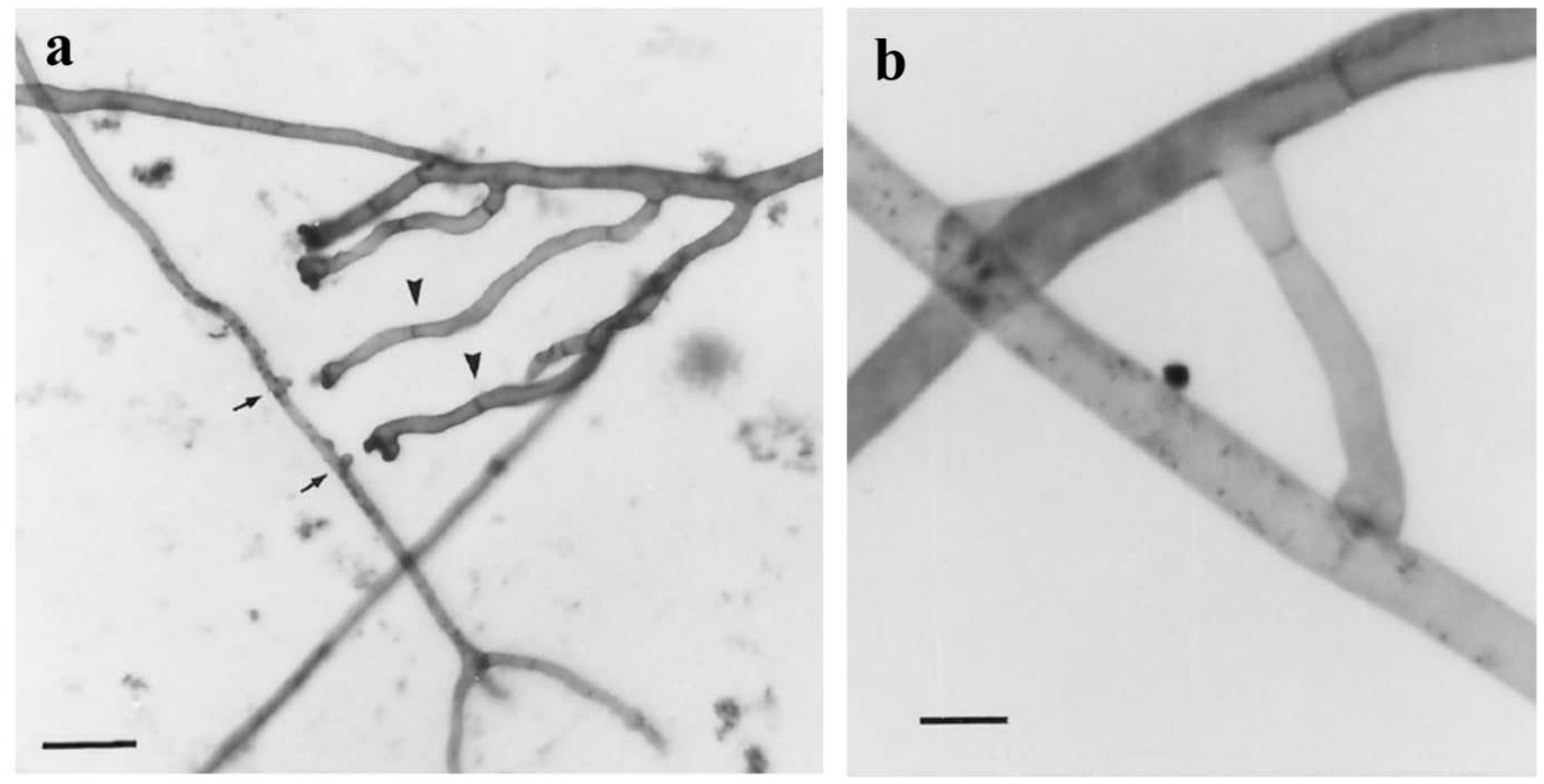

Figure 7. Evidence of pre-fusion vegetative incompatibility between isolates of Glomus mosseae. Note swelling, retraction and formation of septa before fusion of hyphae. From Giovannetti et al. (2003). Bars $=35 \mu \mathrm{m}$ in a; $9 \mu \mathrm{m}$ in b. 


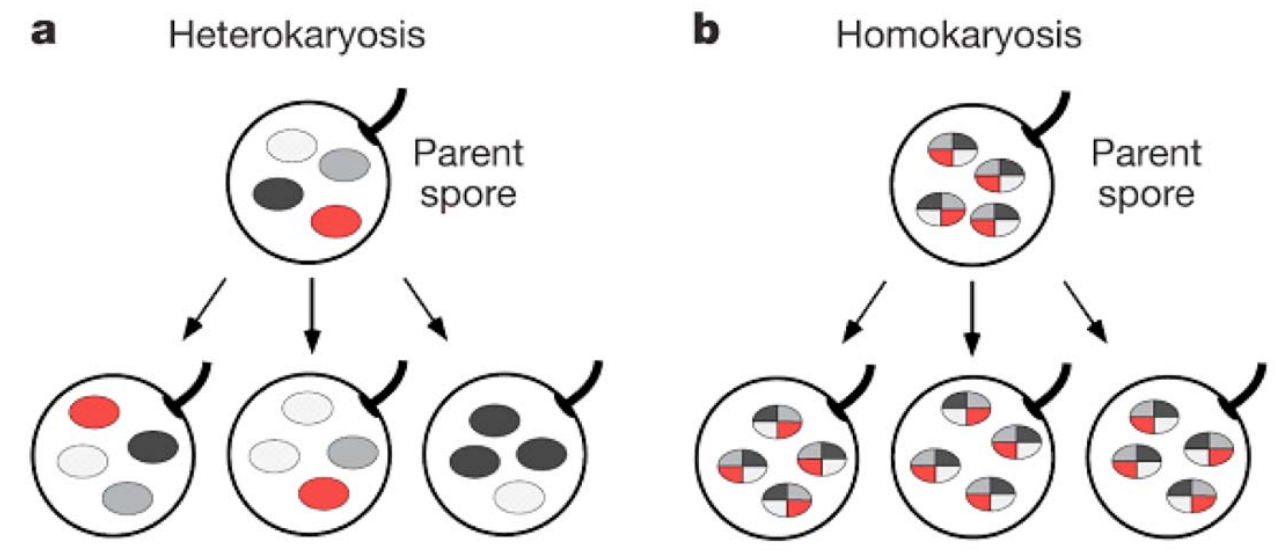

Figure 8. Sorting of genetic variation in AMF according to contrasting (a) heterokaryotic and (b) homokaryotic models. From Pawlowska \& Taylor (2004). 


\section{References}

Aanen DK, Debets AJM, de Visser JAGM, Hoekstra RF. 2008. The social evolution of somatic fusion. Bioessays 30: 1193-1203.

Bécard G, Fortin JA. 1988. Early events of vesicular arbuscular mycorrhiza formation on Ri TDNA transformed roots. New Phytologist 108: 211-218.

Bever JD, Wang M. 2005. Arbuscular mycorrhizal fungi - Hyphal fusion and multigenomic structure. Nature 433: E3-E4.

Biella S, Smith ML, Aist JR, Cortesi P, Milgroom MG. 2002. Programmed cell death correlates with virus transmission in a filamentous fungus. Proceedings of The Royal Society of London Series B - Biological Sciences 269: 2269-2276.

Bonfante P, Perotto S. 1995. Strategies of arbuscular mycorrhizal fungi when infecting host plants. New Phytologist 130: 3-21.

Boucherie H, Bernet J. 1978. Protoplasmic incompatibility and self-lysis in Podospora anserina: enzymes associated with cell destruction. Canadian Journal of Botany 56: 2171-2176.

Bursch W. 2001. The autophagosomal-lysosomal compartment in programmed cell death. Cell Death and Differentiation 8: 569-581.

Buss LW. 1983. Somatic variation and evolution. Paleobiology 9: 12-16.

Cardenas Flores A, Draye X, Bivort C, Cranenbrouck S, Declerck S. 2010. Impact of multispores in vitro subcultivation of Glomus sp. MUCL 43194 (DAOM 197198) on vegetative compatibility and genetic diversity detected by AFLP. Mycorrhiza 20: 415-425.

Chevanne D, Bastiaans E, Debets A, Saupe SJ, Clavé C, Paoletti M. 2009. Identification of the het-r vegetative incompatibility gene of Podospora anserina as a member of the fast evolving HNWD gene family. Current Genetics 55: 93-102.

Coppin E, Debuchy R, Arnaise S, Picard M. 1997. Mating types and sexual development in filamentous Ascomycetes. Microbiology and Molecular Biology Reviews 61: 411-428.

Croll D, Giovannetti M, Koch AM, Sbrana C, Ehinger M, Lammers PJ, Sanders IR. 2009. Nonself vegetative fusion and genetic exchange in the arbuscular mycorrhizal fungus Glomus intraradices. New Phytologist 181: 924-937.

de la Providencia IE, de Souza FA, Fernandez F, Delmas NS, Declerck S. 2005. Arbuscular mycorrhizal fungi reveal distinct patterns of anastomosis formation and hyphal healing mechanisms between different phylogenic groups. New Phytologist 165: 261-271. 
de Souza FA, Declerck S. 2003. Mycelium development and architecture, and spore production of Scutellospora reticulata in monoxenic culture with Ri T-DNA transformed carrot roots. Mycologia 95: 1004-1012.

Debets AJM, Griffiths AJF. 1998. Polymorphism of het genes prevents resource plundering in Neurospora crassa. Mycological Research 102: 1343-1349.

Garriock ML, Peterson RL, Ackerley CA. 1989. Early stages in colonization of Allium porrum (leek) roots by the vesicular-arbuscular mycorrhizal fungus, Glomus versiforme. New Phytologist 112: 85-92.

Gianinazzi S. 1991. Vesicular-arbuscular (endo-) mycorrhizas - cellular, biochemical and genetic-aspects. Agriculture Ecosystems and Environment 35: 105-119.

Giovannetti M, Azzolini D, Citernesi AS. 1999. Anastomosis formation and nuclear and protoplasmic exchange in arbuscular mycorrhizal fungi. Applied and Environmental Microbiology 65: 5571-5575.

Giovannetti M, Fortuna P, Citernesi AS, Morini S, Nuti MP. 2001. The occurrence of anastomosis formation and nuclear exchange in intact arbuscular mycorrhizal networks. New Phytologist 151: 717-724.

Giovannetti M, Sbrana C, Avio L, Strani P. 2004. Patterns of below-ground plant interconnections established by means of arbuscular mycorrhizal networks. New Phytologist 164: 175-181.

Giovannetti M, Sbrana C, Strani P, Agnolucci M, Rinaudo V, Avio L. 2003. Genetic diversity of isolates of Glomus mosseae from different geographic areas detected by vegetative compatibility testing and biochemical and molecular analysis. Applied and Environmental Microbiology 69: $616-624$.

Glass NL, Dementhon K. 2006. Non-self recognition and programmed cell death in filamentous fungi. Current Opinion in Microbiology 9: 553-558.

Glass NL, Jacobson DJ, Shiu PKT. 2000. The genetics of hyphal fusion and vegetative incompatibility in filamentous ascomycete fungi. Annual Review of Genetics 34: 165-186.

Glass NL, Kaneko I. 2003. Fatal attraction: Nonself recognition and heterokaryon incompatibility in filamentous fungi. Eukaryotic Cell 2: 1-8.

Glass NL, Kuldau GA. 1992. Mating type and vegetative incompatibility in filamentous ascomycetes. Annual Review of Phytopathology 30: 201-24.

Glass NL, Rasmussen C, Roca MG, Read ND. 2004. Hyphal homing, fusion and mycelial interconnectedness. Trends in Microbiology 12: 135-141. 
Gregory PH. 1984. The fungal mycelium: an historical-perspective. Transactions of the British Mycological Society 82: 1-11.

Harrison MJ. 1997. The arbuscular mycorrhizal symbiosis: An underground association. Trends in Plant Science 2: 54-60.

Harrison MJ. 1999. Molecular and cellular aspects of the arbuscular mycorrhizal symbiosis. Annual Review of Plant Physiology and Plant Molecular Biology 50: 361-389.

Hickey PC, Jacobson DJ, Read ND, Glass NL. 2002. Live-cell imaging of vegetative hyphal fusion in Neurospora crassa. Fungal Genetics and Biology 37: 109-119.

Jakobsen I, Abbott LK, Robson AD. 1992. External hyphae of vesicular-arbuscular mycorrhizal fungi associated with Trifolium subterraneum L. 1. Spread of hyphae and phosphorus inflow into roots. New Phytologist 120: 371-380.

Jany JL, Pawlowska TE. 2010. Multinucleate spores contribute to evolutionary longevity of asexual Glomeromycota. American Naturalist 175: 424-435.

Marek SM, Wu J, Glass NL, Gilchrist DG, Bostock RM. 2003. Nuclear DNA degradation during heterokaryon incompatibility in Neurospora crassa. Fungal Genetics and Biology 40: 126-137.

McCabe PM, Gallagher MP, Deacon JW. 1999. Microscopic observation of perfect hyphal fusion in Rhizoctonia solani. Mycological Research 103: 487-490.

Mikkelsen BL, Rosendahl S, Jakobsen I. 2008. Underground resource allocation between individual networks of mycorrhizal fungi. New Phytologist 180: 890-898.

Newhouse JR, MacDonald WL. 1991. The ultrastructure of hyphal anastomoses between vegetatively compatible and incompatible virulent and hypovirulent strains of Cryphonectria parasitica. Canadian Journal of Botany 69: 602-614.

Paoletti M, Castroviejo M, Begueret J, Clave C. 2001. Identification and characterization of a gene encoding a subtilisin-like serine protease induced during the vegetative incompatibility reaction in Podospora anserina. Current Genetics 39: 244-252.

Paoletti M, Saupe SJ, Clave C. 2007. Genesis of a fungal non-self recognition repertoire. Plos One 2: e283.

Paoletti M, Clave C. 2007. The fungus-specific HET domain mediates programmed cell death in Podospora anserina. Eukaryotic Cell 6: 2001-2008.

Paoletti M, Saupe SJ. 2009. Fungal incompatibility: Evolutionary origin in pathogen defense? Bioessays 31: 1201-1210. 
Pawlowska TE, Taylor JW. 2004. Organization of genetic variation in individuals of arbuscular mycorrhizal fungi. Nature 427: 733-737.

Pinan-Lucarré R, Paoletti M, Dementhon K, Coulary-Salin B, Clave C. 2003. Autophagy is induced during cell death by incompatibility and is essential for differentiation in the filamentous fungus Podospora anserina. Molecular Microbiology 47: 321-333.

Pittenger TH, Brawner TG. 1961. Genetic control of nuclear selection in Neurospora heterokaryons. Genetics 46: 1645-1663.

Saupe S, Descamps C, Turcq B, Begueret J. 1994. Inactivation of the Podospora anserina vegetative incompatibility locus het-c, whose product resembles a glycolipid transfer protein, drastically impairs ascospore production. Proceedings of the National Academy of Sciences of the United States of America 91: 5927-5931.

Saupe S, Turcq B, Begueret J. 1995. Sequence diversity and unusual variability at the het-c locus involved in vegetative incompatibility in the fungus Podospora anserina. Current Genetics 27: 466-471.

Saupe SJ, Glass NL. 1997. Allelic specificity at the het-c heterokaryon incompatibility locus of Neurospora crassa is determined by a highly variable domain. Genetics 146: 1299-1309.

Saupe SJ, Kuldau GA, Smith ML, Glass NL. 1996. The product of the het-C heterokaryon incompatibility gene of Neurospora crassa has characteristics of a glycine-rich cell wall protein. Genetics 143: 1589-1600.

Saupe SJ. 2000. Molecular genetics of heterokaryon incompatibility in filamentous ascomycetes. Microbiology and Molecular Biology Reviews 64: 489-502.

Saupe SJ. 2007. A short history of small s a prion of the fungus Podospora anserina. Prion 1: $110-115$.

Sbrana D, Fortuna P, Giovannetti M. 2010. Plugging into the network: belowground connections between germlings and extraradical mycelium of arbuscular mycorrhizal fungi. Mycologia. doi:10.3852/10-125.

Shiu PKT, Glass NL. 1999. Molecular characterization of tol, a mediator of mating-typeassociated vegetative incompatibility in Neurospora crassa. Genetics 151:545-55.

Smith ML, Micali OC, Hubbard SP, Mir-Rashed N, Jacobson DJ, Glass NL. 2000. Vegetative incompatibility in the het-6 region of Neurospora crassa is mediated by two linked genes.

Genetics 155: 1095-1104.

Smith SE, Read DJ. 2008. Mycorrhizal symbiosis. Cambridge, UK: Academic Press. 
Tommerup IC. 1988. The vesicular arbuscular mycorrhizas. Advances in Plant Pathology 6: 8191.

Voets L, de la Providencia IE, Declerck S. 2006. Glomeraceae and Gigasporaceae differ in their ability to form hyphal networks. New Phytologist 172: 185-188.

Wang B, Qiu Y-L. 2006. Phylogenetic distribution and evolution of mycorrhizas in land plants. Mycorrhiza 16: 299-363.

Note: all the copyright-protected images used in the bibliographic review were reproduced with permission from the publishers. 


\title{
CHAPTER 1
}

\section{IN SITU ANALYSIS OF ANASTOMOSIS IN REPRESENTATIVE GENERA OF ARBUSCULAR MYCORRHIZAL FUNGI ${ }^{1}$}

\begin{abstract}
Arbuscular mycorrhizal fungi (AMF) form obligate symbiotic associations with plants. As a result, the role of hyphal interactions in the establishment and maintenance of common mycorrhizal networks is poorly understood because of constraints on methods for in situ analysis. We designed a rhizohyphatron that allows the examination of intact mycelia growing from whole mycorrhizal plants. Plants preinoculated with spores were cultivated in a compartment with a connecting tube from which hyphae extend through a fine nylon mesh onto agar-coated slides. Species selected from each of the five AMF genera were used to assess and characterize the anastomosis behavior in the rhizohyphatron. Hyphal networks of Paraglomus occultum, Ambispora leptoticha, Scutellospora heterogama, and Gigaspora gigantea growing on the agar coated slides showed no evidence of hyphal fusion. In contrast, anastomosis occurred in the hyphal networks of Glomus clarum and Glomus intraradices at an average frequency of less than $15 \%$ for both species. The rhizohyphatron developed in this study will provide knowledge of the biology and genetics of self/non-self recognition in AMF and help to better understand Glomeromycotan life history strategies.
\end{abstract}

${ }^{1}$ Purin S, Morton JB. 2011. In situ analysis of anastomosis in representative genera of arbuscular mycorrhizal fungi. Mycorrhiza. doi: 10.1007/s00572-010-0356-9. Reproduced with permission from the publisher. 


\section{Introduction}

Fungi are modular organisms that can grow until the nutritional resources are exhausted (Andrews, 1992). The continuous growth of mycelia permits fungal individuals to explore vast volumes of substrate. However, this hyphal network also poses a challenge to maintaining a continuous flow and exchange of genetic information and cytoplasmic material. A continuity of mycelia is maintained by hyphal fusion (anastomosis) that reconnects sectors that grow apart or become separated by mechanical injuries (Bago et al., 1999; de la Providencia et al., 2005). Hyphal fusions also provide a mechanism for gene flow between genetically distinct individuals within a population (Glass et al., 2000).

Anastomosis has been studied and characterized extensively in species of Ascomycete fungi (Saupe et al., 2000; Smith et al., 2000). After hyphae fuse, protoplasmic intermixing occurs in a confined sector named a heterokaryon (Pontecorvo, 1956; Glass et al., 2000). The expression of vegetative incompatibility genes (vic genes) determines heterokaryon stability. Allelic differences between hyphae trigger the destruction of the heterokaryon and the onset of vegetative incompatibility (for reviews, see Leslie, 1993; Glass \& Kaneko, 2003). Colonies with identical vic alleles are vegetatively compatible, and they maintain a stable heterokaryont mycelium capable of indefinite propagation (Glass \& Dementhon, 2006).

Although the mechanisms and the significance of anastomosis are well understood in Ascomycetes, understanding of hyphal fusions in populations of arbuscular mycorrhizal fungi $(\mathrm{AMF})$ in Glomeromycetes is rudimentary. AMF colonize the roots of many plant species so that mycelial connectivity contributes to common mycorrhizal networks (CMNs) potentially capable of interconnecting roots of the same or different plant species (Giovannetti et al., 2004). Little empirical evidence is available to explain the potential ecological significance of CMNs because of difficulties in making direct in situ observations of growing mycelia. AMF are obligate biotrophs that can only be cultured in the absence of a plant host for a brief period before a mycorrhizal association is established (Giovannetti et al., 1994; 1996). For this reason, AMF hyphal growth and development have been studied mostly in this asymbiotic phase or in monoxenic cultures of transformed roots.

Early studies reveal that different lineages of Glomeromycota exhibit diverse patterns of hyphal interactions, with unique differences in how these fungi behave during a asymbiotic versus a symbiotic phase. In the asymbiotic phase, anastomosis between germination tubes from 
a population of spores does not occur in Gigaspora and Scutellospora species but ranges from $34 \%$ to $90 \%$ in Glomus species (Giovannetti et al., 1999; 2003). In the symbiotic phase, a similar, albeit less dramatic, pattern of anastomosis is observed. The percentage of anastomosis in extraradical hyphae associated with root explants of Gigaspora and Scutellospora species is 4-10\%, while anastomosis in Glomus species reaches 80-100\% (de la Providencia et al., 2005; Voets et al., 2006). By comparison, anastomosis between the hyphae of Glomus mosseae growing from colonized plants is lower, ranging in frequency from $46 \%$ to $78 \%$ (Giovannetti et al., 2001; 2004). Hyphal fusions in the symbiotic phase have not been studied in any other AMF species.

Clearly, the interpretation of anastomosis behavior among external hyphae produced after the establishment of a mycorrhizal symbiosis is complicated by the experimental environment. The in vitro root organ culture system (Bécard \& Fortin, 1988) is atypical because immortalized root explants grow as autonomous organs in a medium with high soluble nutrient levels. The compatibility between fungi and the root host could be altered from the norm, as evidenced by the limited number of species that so far are maintained in culture (Declerck et al., 2005). To overcome these complications as much as possible, a cultivation system was invented in which the behavior and dynamics of hyphae generated from intact mycorrhizal plants growing in a solid substrate could be studied in situ. To test this cultivation system, we evaluated anastomosis within individual populations of a representative species from five glomeromycotan genera.

\section{Materials and methods}

\section{Rhizohyphatron and growing conditions}

A "rhizohyphatron" (Fig. 1) was designed to permit the direct observation of AMF hyphae actively growing in association with roots of intact host plants growing in a solid medium. This cultivation system consisted of two compartments: (1) a plant growth compartment within which mycorrhizal plants were grown for 7 to 8 weeks and (2) a slide compartment to hold two horizontally placed agar-coated glass microscope slides that could be added, removed, or replaced at any time (Fig. 1a). The plant growth compartment consisted of a 20-cm-long PVC tube. When upright, the bottom end of the tube was sealed with a plastic cap having three small holes for water drainage. A 3.4-cm circular opening was cut approximately 
$8.5 \mathrm{~cm}$ from the top of the tube to allow the lateral insertion of the slide compartment. The slide compartment consisted of a 7.0-cm long PVC tube. Plastic supports for slides were cut from $1.0 \times 7.5-\mathrm{cm}$ plastic pot labels and attached to the wall of the tube using $100 \%$ silicon sealant (Silicon II, General Electric Co.). The two supports in each tube were spaced $7.0 \mathrm{~mm}$ apart in the center of the tube (Fig. 1c). The plant and slide compartments were separated by a nylon mesh partition with 41- $\mu$ m openings (Sefar America Inc., Depew, NY, USA) between the plant and slide compartments. The nylon mesh and plastic cap sealed the end of the slide chamber and provided enough resistance so that the slides were in tight contact with the nylon mesh at the juncture of the plant compartment (Fig. 1d). All plastic parts consisted of Schedule 40 PVC pipe fittings, with tubing of 3.4-cm outside diameter.

Before assembly, all components were surface-sterilized by immersion in a $10 \%$ sodium hypochlorite solution for $30 \mathrm{~min}$. Plastic components were washed with distilled water, washed again in $70 \%$ ethanol, and then exposed to ultraviolet light in a laminar flow chamber for approximately $30 \mathrm{~min}$.

To assemble the rhizohyphatron, a 5.0×5.0-cm nylon mesh section was held manually in place at both ends of the slide compartment, with one end inserted into the plant compartment and the other end capped and sealed (Fig. 1d). The top opening of the slide compartment was sealed with transparent tape presterilized by UV irradiation. The plant compartment was filled with Terragreen (Oil Dri Corp., Chicago, IL, USA) that had been premoistened and autoclaved by incubation at $121^{\circ} \mathrm{C}$ for $30 \mathrm{~min}$. Two 10-12-day-old sorghum (Sorghum sudanense L.) seedlings were inoculated with 100-200 spores of a selected fungal isolate (Table 1) pipetted directly and evenly along intertwined roots. Before inoculation, spores were surface-cleaned by sonication and stored at $4{ }^{\circ} \mathrm{C}$ for 2 days. Spores showing any signs of degradation or microbial contamination were removed. After inoculation, seedlings were transplanted into Terragreen in the plant compartment. The slide compartment was covered with a layer of aluminum foil to ensure darkness. This entire protocol was completed in a sterile laminar chamber.

Rhizohyphatrons were placed in a growth room with a light intensity at $320 \mu \mathrm{E} \mathrm{m}^{-2} \mathrm{~s}^{-1}$ for a 14-h period and air temperature ranging from $25^{\circ} \mathrm{C}$ to $27^{\circ} \mathrm{C}$. Plants were watered ad libitum daily with double-distilled deionized water and grown for 5 weeks to allow mycorrhizal development and formation of external hyphae. After 5 weeks, each rhizohyphatron was moved 
to a laminar flow chamber. The cap was removed from the slide compartment, two agar-coated slides were inserted, and the tube resealed with the plastic cap.

To prepare microscope slides for insertion into the rhizohyphatron, a $22 \times 50$-mm cover slip was first affixed to the center of each slide with a $3 \times 3-\mathrm{mm}$ strip of autoclave tape. A cover slip was required to observe and/or measure the details of hyphal interactions under an inverted light microscope. Slides were first autoclaved for $20 \mathrm{~min}$ at $121^{\circ} \mathrm{C}$ and then transferred to a laminar flow chamber, where they were evenly spaced in the bottom of a flat $11.5 \times 20-\mathrm{cm}$ plastic tray, with 14 slides per tray. The slides were covered with a thin, uniform layer of agar by gently pouring $22 \mathrm{ml}$ of autoclaved agar to cover the bottom of the plastic tray. The $1.5 \%$ water agar contained $0.1 \mathrm{mg} \mathrm{kg}^{-1} \mathrm{P}$ (added as $\mathrm{KH}_{2} \mathrm{PO}_{4}$ ) and was adjusted to $\mathrm{pH}$ 5.5. Once the agar solidified, each slide was carefully excised with a sterile dissecting blade and placed immediately into the slide compartment. After the slides were positioned, the slide compartment was capped and the rhizohyphatron transferred to the growth room.

\section{AM fungal isolates}

Globally distributed species representing five of the major genera in Glomeromycota were chosen for this study (Table 1). One productive accession of each species was selected from the International Culture Collection of Vesicular Arbuscular Mycorrhizal Fungi (INVAM; Morton, 1993). Each accession had been started initially from many spores to adequately capture genetic diversity, and then a large sample of each pot culture was used in at least six successive propagation cycles to minimize any possibility of genetic drift. Given the history of these cultures, the possibility of different genetic lineages coexisting in any one of them could not be excluded.

\section{Microscopic evaluation of hyphal contacts}

The spread of AMF external hyphae from the plant growth compartment was monitored by placing the rhizohyphatron under a stereomicroscope and inspecting the top slide through the transparent tape that covered the opening in the slide compartment. Observations began 5 days after the slides were inserted and they were repeated thereafter at 3-day intervals. When a hyphal front had advanced close to midway across the cover slip, the slide and plant compartments were gently separated from each other in a laminar flow chamber (Fig. 2a). Cover slips were detached 
from each slide with forceps and immediately placed under an inverted microscope (Nikon Eclipse, TE2000-S) for examination.

On less than $25 \%$ of the slides, the agar surface became contaminated with bacteria or saprophytic fungi. These units were removed and dismantled. The source of the contaminants was traced to the plant compartment but origins never were identified definitively. Other coatings, such as Millipore membranes, were tested in attempts to reduce contamination. However, hyphae failed to grow from the plant compartment onto these membranes.

Hyphae present on cover slips were verified as being of AMF origin based on the structures of each species that typically formed concomitant with hyphal development, such as spores, auxiliary cells, or saccules. To facilitate the observation and identification of hyphal contacts, each cover slip was overlaid on a $1.0 \times 1.0-\mathrm{mm}$ grid. The hyphal front served as the base reference point (Fig. 2b). Hyphal contacts were evaluated in alternate columns distal to the hyphal front for a distance of $11 \mathrm{~mm}$. Therefore, the columns in the grid were evaluated at 1, 3, 5, 7, 9, and $11 \mathrm{~mm}$ from the hyphal front (Fig. 2b). Preliminary observations had indicated that most variations in hyphal anastomosis occurred in this region (data not shown). Within each column, alternate squares were examined, totaling an area of $11 \mathrm{~mm}^{2}$ per column. This approach was used for the isolates of Ambispora, Glomus, and Paraglomus species. Because hyphal contacts were much less frequent among hyphae formed by the isolates of Gigaspora gigantea and Scutellospora heterogama, all columns and all squares in each column were examined up to $11 \mathrm{~mm}$ from the hyphal front. One rhizohyphatron and two cover slips were evaluated per AMF isolate.

Hyphal contacts were classified as "compatible," "incompatible," or "no recognition" based on published descriptions of vegetative interactions in AMF. Compatible hyphal interactions were identified when interacting hyphae formed bridges with complete wall fusion and protoplasmic continuity (Giovannetti et al., 1999; 2001; 2004). Interactions suggestive of incompatibility were recognized when interacting hyphae formed septa, changed shape, or both (Giovannetti et al., 2003). Hyphal contacts were characterized as no recognition when hyphae physically overlapped without any morphological changes (Giovannetti et al., 1999; de la Providencia et al., 2005). The number of hyphal contacts in each category (compatible, incompatible, or no recognition) was divided by the total number of observed hyphal contacts in order to calculate relative frequencies. 


\section{Results}

\section{Rhizohyphatron utility}

The rhizohyphatron provided a suitable environment to conduct in situ observations of hyphal interactions in the symbiotic mycelium of AM fungi. With the exception of Acaulospora, the extraradical mycelium of all species extended into the slide compartment where the hyphal network could be examined microscopically. In most cases, the rhizohyphatron remained free of bacterial and fungal contamination for the duration of an experiment (7-8 weeks). Fungal contamination occurred in less than $25 \%$ of rhizohyphatrons and varied in both composition and abundance depending on the fungal isolate used as inoculum. Fungal contaminants always grew from the plant compartment.

\section{Patterns of hyphal interactions in AM fungi}

For all AMF species, the slide compartment was opened and cover slips were removed for microscopic analysis when the mycelia had grown an average distance of $40 \mathrm{~mm}$ on the agar surface. The mycelia of Ambispora leptoticha, Glomus clarum, and Glomus intraradices reached this distance within 7 days after the slides were placed in the rhizohyphatron. A similar distance for mycelial growth of other species was observed at 14 days. The mycelia of A. leptoticha, $G$. clarum, G. intraradices and Paraglomus occultum produced spores branching from older hyphae, especially near the nylon mesh partition separating the plant and slide compartments. The hyphae of G. gigantea and S. heterogama did not produce any spores, but they did form abundant auxiliary cells. Fast and bidirectional protoplasmic streaming was observed in the hyphae of A. leptoticha, G. clarum, and G. intraradices. In the hyphae of other species, bidirectional streaming was observed but the rate of streaming appeared to be markedly slower.

Evidence of hyphal fusion was observed only in the Glomus species analyzed. The hyphae of both $G$. clarum and $G$. intraradices fused by producing bridge-like structures. Bridges shorter than $50 \mu \mathrm{m}$ were classified as "short bridges" (Fig. 3a, b), and bridges longer than $50 \mu \mathrm{m}$ were classified as "long bridges" (Fig. 3c). Protoplasm flow between two hyphae via hyphal bridges confirmed mycelium continuity. Short and long bridges formed with similar frequency between hyphae of both species (Table 2). 
A unique anastomosis behavior was observed in G. clarum that did not involve hyphal tips or projections. Cell wall and membrane fusion occurred between some hyphae growing in such close proximity to each other that no bridges were formed (Fig. 3d). Protoplasmic streaming was observed in regions where hyphal walls in direct contact had dissolved, thus confirming that anastomosis had occurred.

Three morphological changes in interacting hyphae were hypothesized to indicate vegetative incompatibility: (1) septation, (2) change in shape, and (3) septation accompanied by change in shape (Table 2). Septation without any change in shape occurred most frequently in the hyphae of most of the AMF species tested and it often was localized near the tips and in coarser hyphae. Septation was the only indication of vegetative incompatibility among hyphae of G. gigantea and S. heterogama. For all AMF species, mycelial compartments created by septa were devoid of cytoplasm (Fig. 4a).

Changes in the shape of the hyphae were observed in all genera except Gigaspora and Scutellospora, regardless of the occurrence of septa formation. When a hyphal tip or lateral projection contacted a putative incompatible hypha, the morphology of the former was altered (Fig. 4b). Tips became swollen with lateral expansions, and anastomosis did not occur between the interacting hyphae (Fig. 4b). This response occurred most frequently as indicative of vegetative incompatibility in the hyphae of A. leptoticha and G. intraradices. In some instances, septation occurred with a change in hyphal shape and the flow of cytoplasm became blocked (Fig. 4c).

Hyphal contacts classified as no recognition occurred most frequently among the hyphae of all AMF species studied. Hyphae established physical contact with each other, but no evidence of anastomosis, septation, or change of shape was discernible (Fig. 4d). For $P$. occultum, all hyphal interactions were characterized by no recognition.

The average frequency of compatible hyphal contacts did not exceed $15 \%$ among the two species in which anastomosis occurred (Table 3), ranging from 6.7\% in G. clarum to $13.9 \%$ in $G$. intraradices. The frequency of incompatible contacts ranged from $7 \%$ to $32 \%$ among the six species studied. Among all AMF species, 61-100\% of hyphae in physical contact with each other showed no evidence of recognition (Table 3). 


\section{Discussion}

The rhizohyphatron designed for this study represents a unique tool for the in situ analysis of AMF external mycelia. The components of this system are easy to mass produce, and maintenance is straightforward. Slides can be inserted in the rhizohyphatron at any time after the establishment of root colonization so that the mycelium can be evaluated at any stage of the symbiosis or age of the host plant.

In an open system such as this one, contamination always is the most serious concern. Less than $25 \%$ of rhizohyphatrons showed evidence of either fungal or bacterial contamination, but the absence of any pattern suggested that the most likely source was spores in the inocula. Even though spores were incubated for 2 days to permit the growth of any internal parasites, some still could have escaped detection at the time of inspection. The 2-day incubation period used in this study to detect these contaminants was probably insufficient for some spore populations. Spore germination assays and inoculation of axenic root organ cultures with a range of isolates in INVAM indicate that even the most visually clean spores may harbor internal fungal or bacterial propagules, and these sometimes are not detected without a longer incubation (results not shown). In this study, the rhizohyphatrons were maintained in a room were several other pot cultures are maintained and so some contamination also from the air cannot be excluded.

The rhizohyphatron successfully allowed the anastomosis behavior in symbiotic hyphal networks to be studied for the first time in ancestral AMF species. The rhizohyphatron may also provide a useful approach in studying hyphal architecture, rates of hyphal development, and other variables in situ.

Anastomosis has been studied previously in fungi with membership in Glomus group A, Gigaspora, and Scutellospora. Our study expanded the analysis of anastomosis to include $A$. leptoticha and P. occultum, two species in ancestral clades (Redecker \& Raab, 2006). In addition to the species listed in Table 1, we also tested Acaulospora morrowiae (INVAM accession number WV220), but this species did not produce hyphae in the rhizohyphatron. Acaulospora species, in general, tend to be unpredictable even in standard pot culture environments (personal observation). Different species and growth media will need to be screened to resolve the compatibility issues in this genus. 
The frequency of hyphal fusions in Gigaspora and Scutellospora has been reported previously to average less than $10 \%$ (de la Providencia et al., 2005; Voets et al., 2006) or not to occur at all (Giovannetti et al., 1999). The results of this study support the findings of the latter study. The absence of hyphal fusion in Archaeospora and Paraglomus suggests that anastomosis is a derived trait present at least in the Glomus group A clade (Schüßler et al., 2001). In other fungi, anastomosis is important for colony formation to homogenize water and nutrient distribution in the mycelium and to reconstitute mycelium continuity when hyphae are injured (Gregory, 1984). However, hyphal fusions are rare or absent when a population harbors deleterious cytoplasmic elements, so their transmission among individuals is avoided (Biella $e t$ al., 2002). However, without knowledge of AMF genomes, it is difficult to explain the occurrence of anastomosis in one major clade only. A similar behavior may be found in other Glomus clades (B and C), but those species have not yet been tested.

Evidence suggests that anastomosis in Glomus mediates the underground resource allocation between plants by the formation of CMNs (Mikkelsen et al., 2008) and is important for hyphae-healing mechanisms (de la Providencia et al., 2005). These benefits would be of positive value to all glomeromycotan fungi and would not be restricted to Glomus group A. Whatever historical events or processes involved in the emergence and heritability of anastomosis cannot be inferred from data available at this time.

The dominant type of compatible hyphal interactions observed in Glomus species was the formation of hyphal bridges, which have also been described in some ascomycetes. In Neurospora crassa, Tuber borchii, and Beauveria bassiana, for example, short bridges are produced mainly when hyphae grow parallel to each other and in close proximity (Kawamoto \& Aizawa, 1989; Hickey et al., 2002; Marek et al., 2003; Sbrana et al., 2007). Long bridges are observed as well in some ascomycete species but at frequencies lower than those measured for short bridges (Ainsworth \& Rayner, 1986). The occurrence of long bridges is hypothesized to involve signaling molecules required for attraction (Hickey et al., 2002). In this study, an evidence of the homing of hyphal branches towards each other before fusion supports this hypothesis. In some Glomus species, the existence of signaling molecules might be important because short and long bridges were formed with similar frequency.

All fusion events between vegetative hyphae reported to date involve homing of at least one hyphal tip, such as observed in the formation of bridges and "tip-to-side" fusions or pegs 
(Ainsworth \& Rayner, 1986; Aylmore \& Todd, 1986; Ainsworth \& Rayner, 1989; Correll et al., 1989; Kues, 2000). The significance of the unique type of anastomosis behavior in G. clarum, where fusion occurred between hyphae growing parallel to each other in close proximity, is not known, but the localization of signaling molecules may intensify the opportunity for fusion events.

Both this study and the one by Giovannetti et al. (2003) indicate that any vegetative incompatibility response among symbiotic external hyphae is expressed prior to a fusion event. Given the coenocytic nature of AMF mycelium and rapid protoplasmic streaming, chemical signals that trigger vegetative incompatibility likely are expressed before anastomosis occurs. Otherwise, the spread of deleterious genetic elements across coenocytic hyphae would occur rapidly and the entire mycelium could be compromised. In contrast, signal recognition in ascomycete and basidiomycete fungi occurs during a post-fusion stage when a heterokaryont mycelium is established. In these fungi, only a small fraction of the hyphal network is compromised when there is allelic incompatibility because plugging of septa would effectively block the spread of deleterious elements (McCabe et al., 1999; Marek et al., 2003).

The frequency of hyphal fusions observed in G. clarum was very low when compared to the results of previous studies on other species in Glomus group A. Anastomosis in the symbiotic mycelia of Glomus hoi, G. mosseae, and Glomus proliferum ranged from $44 \%$ to $100 \%$ (Giovannetti et al., 2001; 2004; de la Providencia et al., 2005; Voets et al., 2006). The type of inoculum likely was not a factor since populations of spores were used in all of these studies as well as the one reported here.

The frequency of anastomosis in G. intraradices was $13.9 \%$, which is much lower than the $88 \%$ to $100 \%$ observed by de la Providencia et al. (2005) and Voets et al. (2006), respectively. However, the frequency of anastomosis could vary greatly between ecologically and geographically disjunct populations of a species, as has been observed in some basidiomycetes (Sbrana et al., 2007). Differences in cultivation systems may also influence the rate of hyphal anastomosis because of the variation in physiological processes and/or gene expression. Anastomosis in G. intraradices has been studied previously only in root organ cultures, which represent a selective and atypical growth environment for AMF. True comparative studies of root organ versus whole plant cultures have yet to be conducted to test the nature and magnitude of those differences. 
An overall anastomosis frequency of less than $15 \%$ in glomeromycete fungi is low compared to that of fungal species in other groups. Isolates of the basidiomycete Sclerotium rolfsii show no evidence of incompatibility reactions (Punja \& Grogan, 1983). In the ascomycete T. borchii, the percentage of anastomosis ranges between 50 and 88\% (Sbrana et al., 2007). In Rhizoctonia solani, anastomoses occur in more than $50 \%$ of hyphal contacts (Hyakumachi \& Ui, 1987).

The low frequency of anastomosis in AM fungi might be causally linked to the structure of their mycelia, which is coenocytic coupled with rapid protoplasmic streaming (personal observation; Jany \& Pawlowska, 2010). Although little is known about protoplasmic flow within a hyphal network, the absence of septation may allow for the continuous homogenization of cytoplasm in the different parts of a mycelium. Anastomosis then would not impact significantly on the redistribution of nutrients. Gregory (1984) reports that the coenocytic zygomycete fungi lack the ability to anastomose. However, no relationship between the inability to anastomose and mycelium structure or behavior has been established empirically.

In other fungal phyla, anastomosis allows the distribution of extra chromosomal DNA elements across a mycelium (Glass et al., 2000). A majority of studies report the frequency of hyphal fusions per hyphal length or focus on pairing isolates of different vegetative compatibility groups for the transmission of genetic elements such as viruses (Correll et al., 1989; Liu \& Milgroom, 1996). At the present time, it is not known if members of any glomeromycete species harbor plasmids or viruses. Anastomosis possibly plays an important role if AMF are heterokaryonts by compensating the effect of genetic drift (Pawlowska \& Taylor, 2004; Bever \& Wang, 2005), but details of the AMF genetic organization still are not decisively understood.

No sexual stage has been demonstrated in AM fungi; so, the only means for gene transfer among individuals is through the vegetative fusion of mycelia (Croll et al., 2009). Low percentages of anastomosis would suggest that the gene flow among "individuals" of AM fungi also likely is low. Other mechanisms must be active to provide genetic variability and compensate for genetic isolation.

Even though single isolate cultures were used in this study, inocula did not originate from a single spore. Therefore, the symbiotic hyphal interactions observed in all species at present must be interpreted within the context of a population with the possibility that more than one 
genotype was present. Even so, the absence of anastomosis in all of the genera tested except Glomus group A remains enigmatic.

\section{Acknowledgements}

The authors wish to thank Bill Wheeler and Robert Bills for the help in building and maintaining the rhizohyphatrons. We would like to thank Dr. J. Yao for the use of the inverted microscope in his laboratory. We are especially indebted to Dr. Teresa Pawlowska for insightful guidance and mentorship. Funding was provided by a Fulbright/CAPES Ph.D. scholarship to Sonia Purin and NSF grant DEB-0649341 to Joseph Morton. 
Table 1. Accessions obtained from the INVAM for the in situ evaluation of anastomosis.

\begin{tabular}{lcc}
\hline \multicolumn{1}{c}{ Species } & Accession code & Origin - contributor \\
\hline Ambispora leptoticha & CR312 & Costa Rica - L. Aldrich-Wolfe \\
Gigaspora gigantea & MN414D & Minnesota, USA - F. Pfleger \\
Glomus clarum & WV101 & West Virginia, USA - J. B. Morton \\
Glomus intraradices & ON201B & Ontario, Canada - T. McGonigle \\
Paraglomus occultum & WY112A & Wyoming, USA - D. Watson \\
Scutellospora heterogama & SN722 & Singapore - I. Louis \\
\hline
\end{tabular}


Table 2. Type and number of hyphal contacts indicative of compatibility and/or incompatibility of symbiotic external hyphae from the six glomeromycotan species tested, as measured on a $1 \times 1$ $\mathrm{mm}$ grid overlaying an agar-coated slide.

\begin{tabular}{lcccc}
\hline $\begin{array}{c}\text { Vegetative compatibility } \\
\text { in species: }\end{array}$ & $\begin{array}{c}\text { Short } \\
\text { bridges }\end{array}$ & $\begin{array}{c}\text { Long } \\
\text { Bridges }\end{array}$ & $\begin{array}{c}\text { Parallel growth } \\
\text { and fusion }\end{array}$ & $\begin{array}{c}\text { Total compatible } \\
\text { contacts }\end{array}$ \\
\hline Glomus clarum & 5 & 4 & 2 & 11 \\
Glomus intraradices & 14 & 12 & 0 & 26 \\
\hline \multicolumn{1}{c}{ Vegetative } & Septation & Change & Septation + & Total incompatible \\
\multicolumn{1}{c}{ incompatibility in } & & in shape & change in shape & contacts \\
$\quad$ species: & 5 & 10 & & 16 \\
\hline Ambispora leptoticha & 38 & 0 & 0 & 38 \\
Gigaspora gigantea & 44 & 4 & 5 & 53 \\
Glomus clarum & 3 & 7 & 4 & 25 \\
Glomus intraradices & 25 & 0 & 0 & 0 \\
Scutellospora heterogama & 0 & 0 & 0 & 14 \\
Paraglomus occultum & & & & \\
\hline
\end{tabular}


Table 3. Total number of hyphal contacts observed in the six glomeromycotan species tested. The hyphal contacts were classified as "compatible," "incompatible," and "no recognition." The frequency of contacts (calculated as a percentage) was determined from the number of hyphal contacts in each category divided by the total number of contacts.

\begin{tabular}{lcccc}
\hline \multicolumn{1}{c}{ Species } & $\begin{array}{c}\text { Number of } \\
\text { contacts }\end{array}$ & $\begin{array}{c}\text { Compatible } \\
\text { contacts } \\
\mathbf{( \% )}\end{array}$ & $\begin{array}{c}\text { Incompatible } \\
\text { contacts } \\
(\boldsymbol{\%})\end{array}$ & $\begin{array}{c}\text { No } \\
\text { recognition }\end{array}$ \\
\hline Ambispora leptoticha & 149 & 0 & 10.7 & 89.3 \\
Gigaspora gigantea & 247 & 0 & 15.4 & 84.6 \\
Glomus clarum & 165 & 6.7 & 32.1 & 61.2 \\
Glomus intraradices & 187 & 13.9 & 7.5 & 78.6 \\
Paraglomus occultum & 118 & 0 & 0 & 100.0 \\
Scutellospora heterogama & 189 & 0 & 13.2 & 86.8 \\
\hline
\end{tabular}



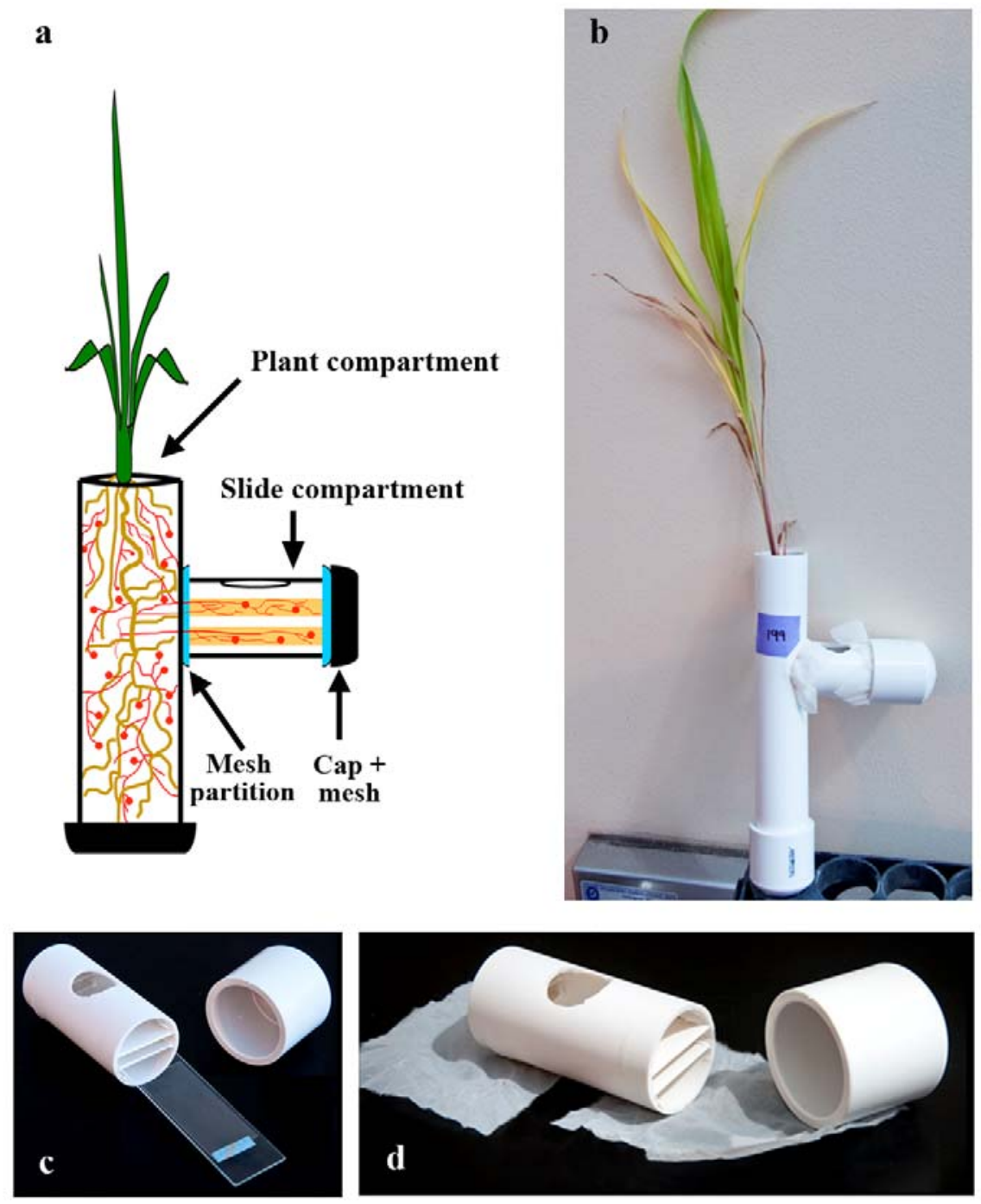

Figure 1. Design of a rhizohyphatron used to evaluate interactions among external hyphae of arbuscular mycorrhizal fungi originating from symbiotic mycorrhizal plants. a) Illustration of the assembled unit: a vertical plant compartment containing preinoculated mycorrhizal seedlings, a lateral slide compartment for the placement of two agar-coated glass slides, a nylon mesh with 41- $\mu \mathrm{m}$ openings so that only hyphae from the plant compartment are able to grow into the slide compartment, and a nylon mesh-and-cap combination to keep the slides in place and seal the slide compartment. b) An assembled unit containing two 4-week-old mycorrhizal sorghum plants. c) The slide compartment consisting of a $3.4 \times 7-\mathrm{cm}$ PVC tube with a centered $3.3-\mathrm{cm}$ opening to observe and monitor hyphal growth on top slide and plastic supports for glass slides made from pot labels and affixed with $100 \%$ silicon. d) End cap and nylon mesh with $40-\mu \mathrm{m}$ openings to cover each end of the pictured slide compartment. 

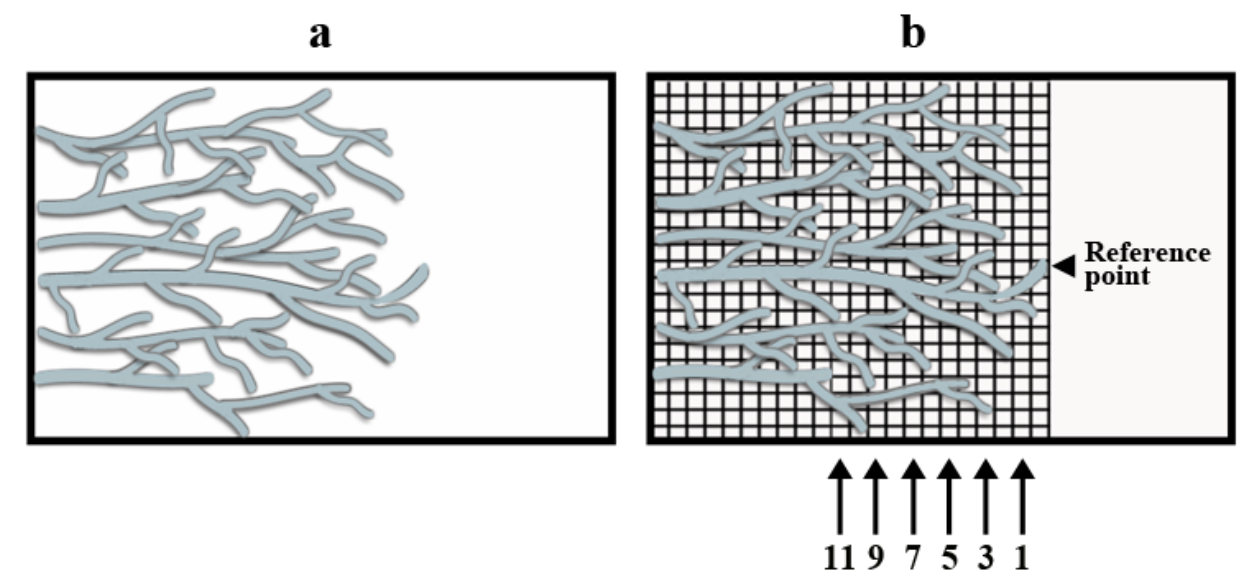

Figure 2. a) Illustration of AMF mycelia growing on a cover slip coated with agar. b) cover slip overlaying a $1 \times 1-\mathrm{mm}$ grid to evaluate anastomosis. The hyphal tip most distal from the side of the cover slip nearest the plant compartment was identified as the reference point for measurements. Hyphal contacts were evaluated in alternate squares of 1-mm-wide columns (labeled 1-11) starting at the reference edge. 

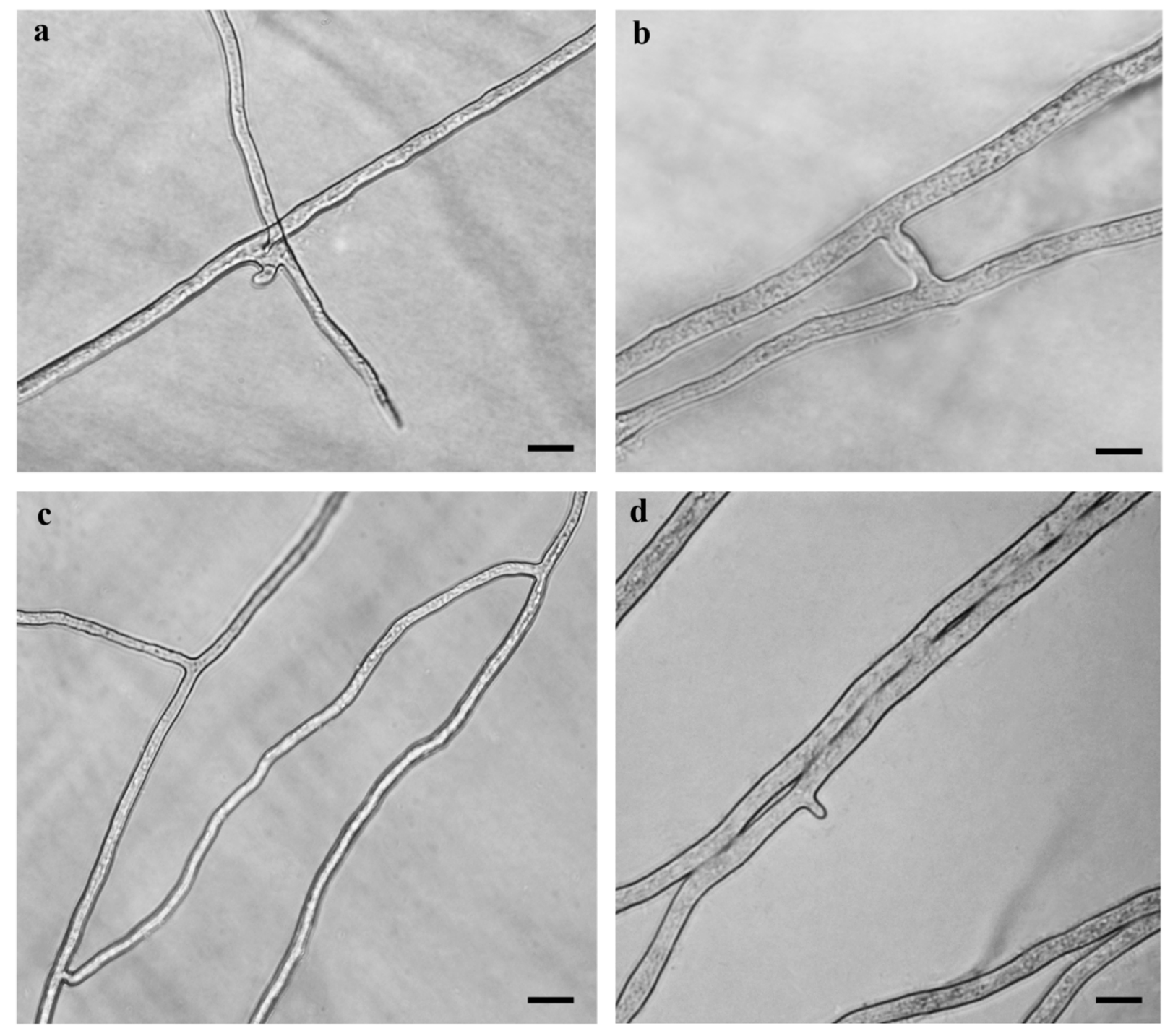

Figure 3. Hyphal contacts in the two Glomus species that produced compatible hyphal fusions (anastomosis). a) and b) Formation of a short bridge between the hyphae of G. intraradices in close proximity. c) Formation of a long bridge between the more distant hyphae of $G$. intraradices. d) Fusion of walls of G. clarum hyphae in direct contact with each other. Bars $=25$ $\mu \mathrm{m}$. 

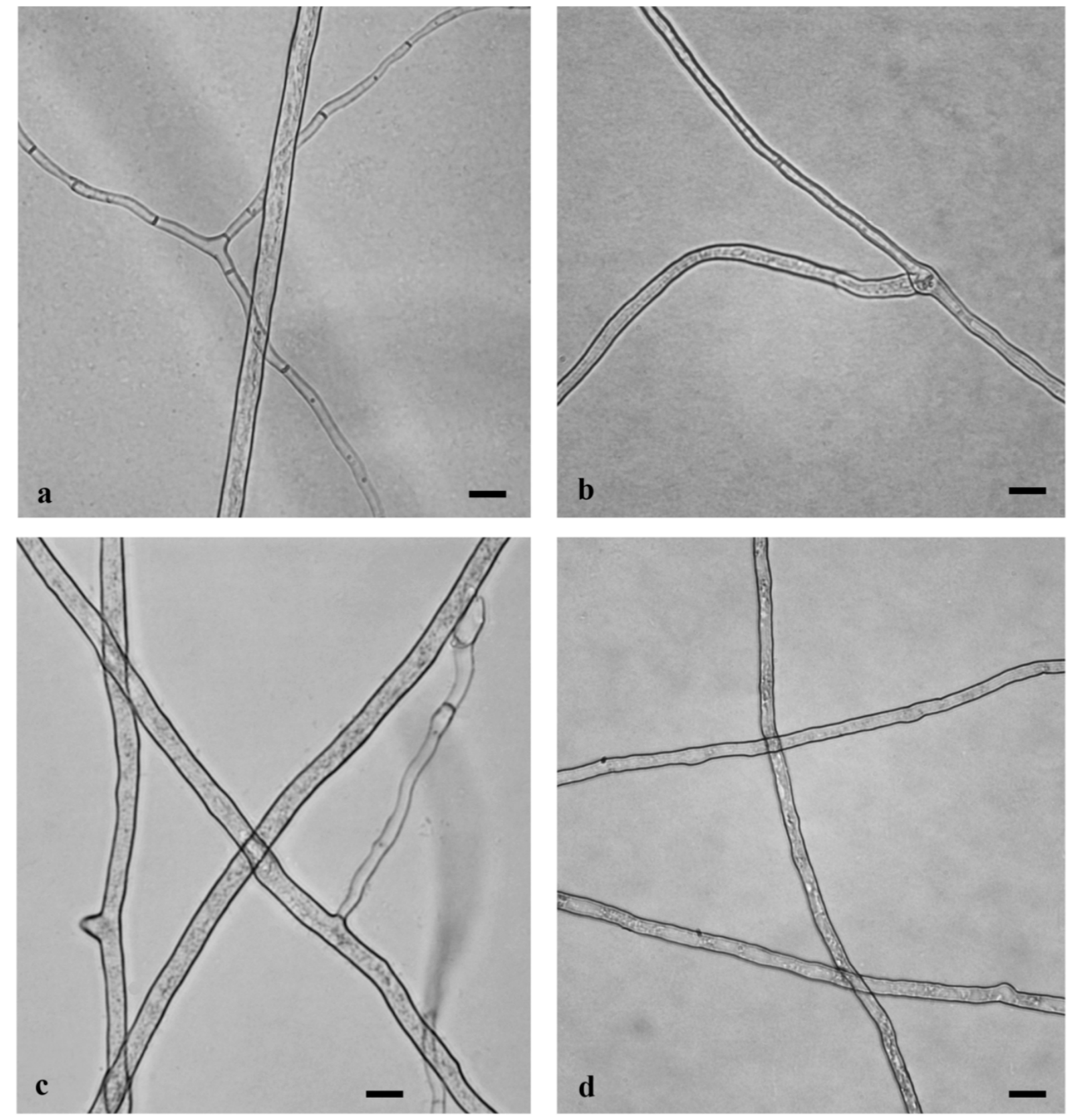

Figure 4. Hyphal contacts suggestive of vegetative incompatibility in two AMF species. a) Septa formation in a hypha of G. clarum. b) Change in shape of a hyphal branch of G. clarum. c) Septation and change in shape of a hyphal branch of $G$. clarum. d) No recognition between the hyphae of $G$. gigantea. Bars $=25 \mu \mathrm{m}$. 


\section{References}

Ainsworth AM, Rayner ADM. 1986. Responses of living hyphae associated with self and nonself fusions in the basidiomycete Phanerochaete velutina. Journal of General Microbiology 132: 191-201.

Ainsworth AM, Rayner ADM. 1989. Hyphal and mycelial responses associated with genetic exchange within and between species of the basidiomycete genus Stereum. Journal of General Microbiology 135: 1643-1659.

Andrews JH. 1992. Fungal life-history strategies. In: Carroll GW, Wicklow DT, eds. The fungal community, its organization and role in the ecosystem. New York: Marcel Dekker, 119-145.

Aylmore RC, Todd NK. 1986. Cytology of self fusions in hyphae of Phanerochaete velutina. Journal of General Microbiology 132: 571-579.

Bago B, Zipfel W, Williams RM, Piché Y. 1999. Nuclei of symbiotic arbuscular mycorrhizal fungi as revealed by in vivo two-photon microscopy. Protoplasma 209: 77-89.

Bécard G, Fortin JA. 1988. Early events of vesicular-arbuscular mycorrhiza formation on Ri TDNA transformed roots. New Phytologist 108: 211-218.

Bever JD, Wang M. 2005. Arbuscular mycorrhizal fungi - Hyphal fusion and multigenomic structure. Nature 433: E3-E4.

Biella S, Smith ML, Aist JR, Cortesi P, Milgroom MG. 2002. Programmed cell death correlates with virus transmission in a filamentous fungus. Proceedings of the Royal Society of London 269: 2269-2276.

Correll JC, Klittich CJR, Leslie JF. 1989. Heterokaryon self-incompatibility in Gibberella fujikuroi (Fusarium moniliforme). Mycological Research 93: 21-27.

Croll D, Giovannetti M, Koch AM, Sbrana C, Ehinger M, Lammers PJ, Sanders IR. 2009. Nonself vegetative fusion and genetic exchange in the arbuscular mycorrhizal fungus Glomus intraradices. New Phytologist 181: 924-937.

de la Providencia IE, de Souza FA, Fernandez F, Delmas NS, Declerck S. 2005. Arbuscular mycorrhizal fungi reveal distinct patterns of anastomosis formation and hyphal healing mechanisms between different phylogenic groups. New Phytologist 165: 261-271.

Declerck S, Séguin S, Dalpé Y. 2005. The monoxenic culture of arbuscular mycorrhizal fungi as a tool for germplasm collections. In: Declerck S, Strullu DG, Fortin A, eds. In vitro culture of mycorrhizas. Berlin: Springer-Verlag, 17-30. 
Giovannetti M, Azzolini D, Citernesi AS. 1999. Anastomosis formation and nuclear and protoplasmic exchange in arbuscular mycorrhizal fungi. Applied and Environmental Microbiology 65: 5571-5575.

Giovannetti M, Fortuna P, Citernesi AS, Morini S, Nuti MP. 2001. The occurrence of anastomosis formation and nuclear exchange in intact arbuscular mycorrhizal networks. New Phytologist 151: 717-724.

Giovannetti M, Sbrana C, Avio L, Strani P. 2004. Patterns of below-ground plant interconnections established by means of arbuscular mycorrhizal networks. New Phytologist 164: $175-181$.

Giovannetti M, Sbrana C, Citernesi AS, Avio L. 1996. Analysis of factors involved in fungal recognition responses to host derived signals by arbuscular mycorrhizal fungi. New Phytologist 133: $65-71$.

Giovannetti M, Sbrana C, Logi C. 1994. Early processes involved in host recognition by arbuscular mycorrhizal fungi. New Phytologist 127: 703-709.

Giovannetti M, Sbrana C, Strani P, Agnolucci M, Rinaudo V, Avio L. 2003. Genetic diversity of isolates of Glomus mosseae from different geographic areas detected by vegetative compatibility testing and biochemical and molecular analysis. Applied and Environmental Microbiology 69: 616-624.

Glass NL, Dementhon K. 2006. Non-self recognition and programmed cell death in filamentous fungi. Current Opinion in Microbiology 9: 553-558.

Glass NL, Jacobson DJ, Shiu PKT. 2000. The genetics of hyphal fusion and vegetative incompatibility in filamentous ascomycete fungi. Annual Review of Genetics 34: 165-186.

Glass NL, Kaneko I. 2003. Fatal attraction: nonself recognition and heterokaryon incompatibility in filamentous fungi. Eukaryotic Cell 2: 1-8.

Gregory PH. 1984. The fungal mycelium: an historical-perspective. Transactions of The British Mycological Society 82: 1-11.

Hickey PC, Jacobson DJ, Read ND, Glass NL. 2002. Live-cell imaging of vegetative hyphal fusion in Neurospora crassa. Fungal Genetics and Biology 37: 109-119.

Hyakumachi M, Ui T. 1987. Non-self-anastomosing isolates of Rhizoctonia solani obtained from fields of sugar-beet monoculture. Transactions of The British Mycological Society 89: 155-159.

Jany JL, Pawlowska TE. 2010. Multinucleate spores contribute to evolutionary longevity of asexual Glomeromycota. American Naturalist 175: 424-435. 
Kawamoto H, Aizawa K. 1989. Morphology of hyphal anastomosis in entomopathogenic fungi, Beauveria bassiana and Beauveria brongniartii. Applied Entomology and Zoology 24: 490-491.

Kues U. 2000. Life history and developmental processes in the basidiomycete Coprinus cinereus. Microbiology and Molecular Biology Reviews 64: 316-353.

Leslie JF. 1993. Fungal vegetative compatibility. Annual Review of Phytopathology 31: 127150.

Liu YC, Milgroom MG. 1996. Correlation between hypovirus transmission and the number of vegetative incompatibility (vic) genes different among isolates from a natural population of Cryphonectria parasitica. Phytopathology 86: 79-86.

Marek SM, Wu J, Glass NL, Gilchrist DG, Bostock RM. 2003. Nuclear DNA degradation during heterokaryon incompatibility in Neurospora crassa. Fungal Genetics and Biology 40: 126-137.

McCabe PM, Gallagher MP, Deacon JW. 1999. Microscopic observation of perfect hyphal fusion in Rhizoctonia solani. Mycological Research 103: 487-490.

Mikkelsen BL, Rosendahl S, Jakobsen I. 2008. Underground resource allocation between individual networks of mycorrhizal fungi. New Phytologist 180: 890-898.

Morton J. 1993. Germ plasm in the International Collection of Arbuscular and VesicularArbuscular Mycorrhizal Fungi (INVAM) and procedures for culture development, documentation and storage. Mycotaxon 48: 491-528.

Pawlowska TE, Taylor JW. 2004. Organization of genetic variation in individuals of arbuscular mycorrhizal fungi. Nature 427: 733-737.

Pontecorvo G. 1956. The parasexual cycle in fungi. Annual Review of Microbiology 10: 393400.

Punja ZK, Grogan RG. 1983. Hyphal interactions and antagonism among field isolates and single-basidiospore strains of Athelia (Sclerotium) rolfsii. Phytopathology 73: 1279-1284.

Redecker D, Raab P. 2006. Phylogeny of the Glomeromycota (arbuscular mycorrhizal fungi): recent developments and new gene markers. Mycologia 98: 885-895.

Saupe SJ, Clavé C, Bégueret J. 2000. Vegetative incompatibility in filamentous fungi: Podospora and Neurospora provide some clues. Current Opinion in Microbiology 3:608-612.

Sbrana C, Nuti MP, Giovannetti M. 2007. Self-anastomosing ability and vegetative incompatibility of Tuber borchii isolates. Mycorrhiza 17: 667-675.

Schüßler A, Schwarzott D, Walker C. 2001. A new fungal phylum, the Glomeromycota: phylogeny and evolution. Mycological Research 105: 1413-1421. 
Smith ML, Micali OC, Hubbard SP, Mir-Rashed N, Jacobson DJ, Glass NL. 2000. Vegetative incompatibility in the het-6 region of Neurospora crassa is mediated by two linked genes. Genetics 155: 1095-1104.

Voets L, de la Providencia IE, Declerck S. 2006. Glomeraceae and Gigasporaceae differ in their ability to form hyphal networks. New Phytologist 172: 185-188. 


\title{
CHAPTER 2
}

\section{ASYMBIOTIC AND SYMBIOTIC HYPHAE OF THE ARBUSCULAR MYCORRHIZAL FUNGUS GLOMUS CLARUM HAVE UNIQUE ROLES IN NON-SELF RECOGNITION ${ }^{2}$}

\begin{abstract}
The life history of arbuscular mycorrhizal fungi (AMF, Glomeromycota) consists of a short asymbiotic phase (germinating spores) and a long symbiotic phase (mycelium associated with a host plant). To date, non-self anastomosis has been studied only in the asymbiotic phase. Two anonymous markers of microsatellite-flanking regions were used to genotype single spore isolates of Glomus clarum from different environments and geographic locations. Patterns of self and non-self recognition as measured by hyphal anastomosis were evaluated in genetically identical and different isolates during both asymbiotic and symbiotic phases. An axenic system was used to examine anastomosis in germinating spores. A rhizohyphatron was used to evaluate anastomosis in symbiotic hyphae. Self anastomosis occurred at similar frequencies (8-38\%) in germinating spores and symbiotic mycelia. Non-self anastomosis was observed only in germinating spores and occurred between both genetically identical and different isolates, at less than $6 \%$ of hyphal contacts. Non-self anastomosis was confined to asymbiotic hyphae of $G$. clarum. This result provides evidence of functional differences between asymbiotic and symbiotic hyphae and that the former provides a short opportunity for gene flow between populations of this AMF species.
\end{abstract}

${ }^{2}$ Submitted for publication in the form of a research manuscript to the Journal "Fungal Biology". 


\section{Introduction}

Arbuscular mycorrhizal fungi (AMF, Glomeromycota) form mutualistic association with a broad range of terrestrial plant species. Like other fungi, AMF are modular organisms and thus have considerable flexibility in growth and behavior, with no defined birth or death. Hyphae within a mycelial network often come in contact with each other and can interact to fuse in the process of anastomosis. Anastomosis can occur in either an asymbiotic phase, when spores germinate to produce one or more germ tubes that elongate and branch in search of roots to colonize, or in a symbiotic phase, where hyphae grow both within and outside a mycorrhizal root. Growth of the fungus in the absence of a host is of brief duration, rarely extending beyond a few weeks (Bécard \& Piché, 1990). External hyphae exist as several forms (Friese \& Allen, 1991) and either grow along root surfaces or expand into the surrounding soil and scavenge for mineral nutrients such as phosphorus and nitrogen (Li et al., 1991; Smith et al., 2001). Self anastomosis occurs within a mycelium produced by one spore and is a universal phenomenon in all fungi studied so far (Glass \& Kuldau, 1992). This process performs the essential function of interconnecting different parts of the mycelium to redistribute water and nutrients within an expanding colony (Gregory, 1984). Non-self anastomosis occurs when mycelia derived from different spores establish contact. This process is advantageous when different alleles other than vic or het co-occur in hyphae with a heterokaryotic nuclear condition. For example, different enzyme forms can increase the ability of a fungus to grow on different nutrient substrates (Jinks, 1952; Caten \& Jinks, 1966). In AMF, non-self anastomosis could help maintain genetic diversity and compensate for the presumably low diversity caused by absence of sexual recombination (Bever \& Wang, 2005). Despite the theoretical advantages of heterokaryon formation, non-self anastomosis occurs with low frequency in most fungi (Aanen et al., 2008). This process is hypothesized to be risky because some populations harbor deleterious cytoplasmic viruses that are transmitted following fusion of hyphae (Liu \& Milgroom, 1996; Cortesi \& Milgroom, 1998). The incidence of aggressive genotypes and parasitic nuclei in some fungal populations also may explain the rarity of non-self anastomosis (Debets \& Griffiths, 1998).

Anastomosis behavior in AMF has been studied mostly by examination of hyphae from germinating spores or mycorrhizal roots that originated from fungal populations of undefined genetic origins (Giovannetti et al., 1999; 2001; 2003; 2004; de la Providencia et al., 2005; Cardenas Flores et al., 2010; Sbrana et al., 2010). Since the AMF organism is of diffuse structure 
and organization, an "individual" or "self" can be defined only by a thallus derived from a single spore or a single spore-derived culture (Croll et al., 2009). The term "non-self" refers to interactions between different individuals, whether they are genetically identical or genetically different (Glass \& Kaneko, 2003; Glass et al., 2004).

Self anastomosis has been studied in only a few AMF species and the patterns appeared to differ both between the asymbiotic and the symbiotic phase as well as among different lineages of glomeromycotan fungi. During the asymbiotic phase, self anastomosis between paired spores can range from 46 to $51 \%$ in Glomus intraradices (Croll et al., 2009). During the symbiotic phase, self anastomosis in G. intraradices and G. proliferum ranges from 80 to $88 \%$ within a symbiotic network, but these numbers decrease to $12-20 \%$ when mycelia are paired (Voets et al., 2006). In contrast, self anastomosis within a mycelium from mycorrhizal roots ranged between 1 and 10\% in Scutellospora and Gigaspora species, but was absent between paired mycelia (de Souza \& Declerck, 2003; de la Providencia et al., 2005).

Non-self anastomosis has been studied only during the asymbiotic phase of the life cycle of one species, $G$. intraradices (Croll et al., 2009). The average frequency of compatible hyphal contacts was $3 \%$, with some evidence suggesting that allelic variation was transmitted to progeny spores. The occurrence of non-self anastomosis during the symbiotic phase remains unknown, even though it comprises most of an AMF life cycle. An experimental constraint has been the absence of a cultivation system that allows for the in situ observation of hyphal interactions between mycelia from paired mycorrhizal plants.

In this study, self and non-self anastomosis were examined in both the asymbiotic phase (germinating spores) and the symbiotic phase (mycorrhizal external hyphae) of Glomus clarum, a species closely related to G. intraradices (Schüßler et al., 2001). We used an axenic Petri dish system (Croll et al., 2009) to measure asymbiotic interactions and a "rhizohyphatron" (Purin \& Morton, 2011) to observe in situ anastomosis between AMF hyphae in the symbiotic phase. "Self" and "non-self" interactions were evaluated between genetically similar and genetically different isolates of $G$. clarum identified using two anonymous genetic markers. We hypothesized that both self and non-self anastomosis can occur during the asymbiotic as well as the symbiotic phase. Contrary to this expectation, non-self anastomosis was observed only during the asymbiotic phase. 


\section{Materials and methods}

\section{Sampling sites and establishment of single spore cultures}

Single spore cultures of G. clarum Nicolson \& Schenck (henceforth defined as isolates) originated from two distinct environments: abandoned coal stripmine sites and pasture mixtures. The mine sites were located in Morgantown, WV (39 38' $\left.1^{\prime \prime} \mathrm{N} 79^{\circ} 57^{\prime} 2^{\prime \prime} \mathrm{W}\right)$, supporting plant communities composed of Andropogon virginicus, Acer rubrum, Crataegus species, Dicranum scoparium, Liriodendron tulipifera, Oxydendrum arboretum, and Solidago species. Soil properties were $\mathrm{pH} 3.0-3.3,2.8-4.2 \mathrm{mg} \mathrm{kg}^{-1}$ Olsen-extractable P, and 229-466 mg kg-1 $\mathrm{Al}$. Pasture mixtures were located near Logan, WV (37 50'54"N 81 $\left.59^{\prime} 16^{\prime \prime} \mathrm{W}\right)$ and Cassville, WV $\left(39^{\circ} 39^{\prime} 43^{\prime \prime} \mathrm{N} 80^{\circ} 03^{\prime} 03^{\prime \prime} \mathrm{W}\right)$, soils of both characterized by $\mathrm{pH}$ of 6.6-6.9, 9.3-12.8 $\mathrm{mg} \mathrm{kg}^{-1}$ Olsenextractable P, and 0.9-1.6 $\mathrm{mg} \mathrm{kg}^{-1}$ Al. Plant communities consisted of Agrostis alba, Achillea millefolium, Festuca eliator, Lolium perenne, Phleum pratense, Plantago lanceolata, Trifolium pratense, and Trifolium repens hydroseeded 5-6 years previously following disturbance. Multiple rhizosphere samples were collected from each location (Fig. 1). Roots and attached soil were collected from the rhizosphere of dominant plants in each mine site and pooled into a $300 \mathrm{~mL}$ sample. Isolates from the International Culture Collection of Arbuscular Mycorrhizal Fungi (INVAM) were used as the source of spores from pasture sites.

AMF spores were extracted from soil by wet-sieving and sucrose gradient centrifugation (Gerdemann \& Nicolson, 1963), washed, collected under a dissecting microscope and surfaced cleaned by sonication in distilled water. Fifteen spores of G. clarum were selected randomly from the population at each location to start single spore cultures. Each spore was pipetted onto intertwined roots of two 10-12 day-old sorghum (Sorghum sudanense L.) seedlings. Plants were transferred to $3.8 \times 21 \mathrm{~cm}\left(150 \mathrm{~cm}^{3}\right) \mathrm{SC} 10$ Supercell "cone-tainers" (Stuewe and Sons, Inc., Corvallis, OR) filled with a mixture of steamed-sterilized soil and Terragreen (Oil Dri Corp., Chicago, IL) in a 1:2 ratio (v/v). Cone-tainers were transferred to a growth room with $420 \mu \mathrm{E} \mathrm{m}{ }^{-2}$ $\mathrm{sec}^{-1}$ light intensity for a 14 hour day period and air temperature ranging from $25-28^{\circ} \mathrm{C}$. Plants were watered ad libitum daily. After 4 months, sporulating cultures were transplanted to $7.5 \times 25$ $\mathrm{cm}\left(600 \mathrm{~cm}^{3}\right)$ D40 “deepots" filled with Terragreen. Sorghum seeds surface-sterilized in 10\% bleach for 10 min and numbering 50-60 were placed in each deepot and covered with a thin layer of Terragreen. Plants were grown for four months under conditions described above. Cultures 
showing evidence of abundant sporulation were air-dried in situ for two weeks, pot contents bagged after removal of plant tops, and stored in sealed zip-loc bags at $4^{\circ} \mathrm{C}$.

\section{Molecular markers}

Microsatellite-flanking regions were used as molecular markers. PCR was performed using select microsatellite repeats known to occur in the genome of some AMF species as primers (Table 1). Genomic DNA of Glomus clarum was extracted from a pooled sample of approximately 2000 spores collected at random from all single spore cultures. Spores were surface-cleaned by sonication in distilled water and transferred to $1.5 \mathrm{~mL}$ Eppendorf tubes containing $0.5 \mathrm{~mm}$ glass beads. Spores were broken using a mini-bead beater (Biospec, USA). Genomic DNA was extracted using a DNeasy plant mini kit (Qiagen) following the manufacturer's protocol.

PCR was performed in a $50-\mu \mathrm{L}$ volume containing $5.0 \mu \mathrm{L}$ of genomic DNA, 1.0 U Taq DNA polymerase (New England Biolabs, USA), $7.0 \mu \mathrm{L} 10 \times \mathrm{PCR}_{\text {buffer, } 3.5 \mathrm{mM} \mathrm{MgCl}}, 1.25$ $\mathrm{mM}$ each of dNTPs, and $1.0 \mu \mathrm{M}$ primer. Amplification conditions were as follows: initial denaturation at $94^{\circ} \mathrm{C}$ for $2 \mathrm{~min} ; 30$ cycles with denaturation at $94^{\circ} \mathrm{C}$ for $30 \mathrm{~s}$, annealing for $30 \mathrm{~s}$, and extension at $72^{\circ} \mathrm{C}$ for $1 \mathrm{~min}$; followed by final extension at $72^{\circ} \mathrm{C}$ for $10 \mathrm{~min}$. The annealing temperature differed for each primer pair (Table 1). Amplicons were visualized by electrophoresis on 1.5\% agarose gel stained with ethidium bromide. Bands were excised and purified using a Gel extraction kit (Qiagen, USA). Purified PCR products were cloned using a cloning kit (Qiagen, USA). Recombinant colonies were grown overnight in $5 \mathrm{~mL}$ of liquid LB medium with ampicillin at $37^{\circ} \mathrm{C}$. Plasmid DNA was purified using a Miniprep kit (Qiagen, USA) and sequenced with M13F and M13R primers using the Big Dye Terminator 3.1 Cycle Sequencing Kit (Applied Biosystems) and ABI 3730XL DNA Analyzer (Applied Biosystems). Sequencing was performed by the West Virginia University Genomics Laboratory. Sequences were edited using Bioedit (Hall, 1999). Sequences were subjected to nucleotide query BlastN search (Altschul et al., 1990) of the National Center for Biotechnology Information (NCBI) databases. Sequences with high similarity to bacterial or fungal DNA sequences were discarded from further analyses. Forward and reverse primers were designed based on remaining sequences deposited in Genbank as accession numbers JF430217-JF430226, excluding the 5' and 3' microsatellite repeats to decrease the risk of no amplification if the number of repeats was 
variable among single spore cultures. Primers listed in Table 2 were designed using the software Primer 3 (Rozen \& Skaletsky, 2000).

\section{Genotyping of single spore cultures}

Two single spore cultures from each location (Fig. 1) were selected for genotyping using newly-designed primers (Table 2). Genomic DNA was extracted from 2000 progeny spores from each culture using a DNeasy plant mini kit (Qiagen). PCR was performed under the same reaction conditions described above, except that a proof-reading enzyme (Tgo DNA polymerase, Roche Applied Science) was used. Amplification conditions were as follows: initial denaturation at $94^{\circ} \mathrm{C}$ for $2 \mathrm{~min} ; 20$ cycles with denaturation at $94^{\circ} \mathrm{C}$ for $30 \mathrm{~s}$, annealing for $60 \mathrm{~s}$, and extension at $72^{\circ} \mathrm{C}$ for $45 \mathrm{~s}$; followed by final extension at $72^{\circ} \mathrm{C}$ for $7 \mathrm{~min}$. Annealing temperature differed for each primer pair (Table 2). PCR products were visualized and purified as described above and sequenced directly. Sequences were aligned in Clustal X (Thompson et al., 1997) and inspected for differences.

One primer pair (Gcla1155-98F/Gcla1155-1058R) failed to amplify DNA of some isolates. Another primer pair (Gcla550-80F/Gcla550-497R) produced non-specific amplification products in some isolates. Only two primer pairs (Gcla690-61F/Gcla690-553R and Gcla65032F/Gcla650-552R) generated sequences that were polymorphic among isolates. The Gcla65032F/Gcla650-552R fragment was highly similar to published sequences from other AMF. A sequence between nucleotide positions 348 and 445 was $94 \%$ similar to one from Glomus intraradices (NCBI accession AC237359.1). Conversely, no similarity was found between the Gcla690-61F/Gcla690-553R fragment and Genbank AMF sequences. All other primer pairs produced monomorphic sequences and thus were uninformative. Sequences obtained from all fungal isolates were deposited in Genbank as accession numbers JF430227-JF430260.

\section{Validation of AMF genotypes}

Single spore isolates 6AmA\#2 and 6AmA\#3 were established as in vitro root organ cultures (Bécard \& Fortin, 1988) to obtain contaminant-free mycelium. Ri T-DNA transformed carrot roots were grown in Petri dishes containing M medium in 3\% Phytagel at pH 6.0 for 1 week at room temperature. Spores were extracted and surface-sterilized with chloramine T and streptomycin/gentamicin sulfate (Bécard \& Fortin, 1988). Approximately 50 spores were placed 
close to root tips in each plate and the plates were stored in the dark at $30^{\circ} \mathrm{C}$ for 8 weeks. The Phytagel was solubilized by adding $10 \mathrm{mM}$ sodium citrate and incubating at $30^{\circ} \mathrm{C}$ for 15 minutes. Mycelia were filtered from the solubilized medium using a $15 \mu \mathrm{M}$ nylon mesh and washed with distilled water. DNA was extracted using a DNeasy plant mini kit (Qiagen). PCR was performed using only primer pairs that generated polymorphic sequences in the genotyping of single spore cultures and the same cycling conditions described previously. Sequences from genomic DNA of both $G$. clarum isolates were identical to those obtained from pot culture samples, thus verifying that amplified DNA originated from G. clarum.

\section{Phylogenetic analyses}

Genetic marker sequences were concatenated and aligned using ClustalX (Thompson et al., 1997). The appropriate model of nucleotide substitution was determined using MODELTEST 3.7 (Posada \& Crandall, 1998). The TrN+I and TrN+G+I (Tamura \& Nei, 1993) models adequately explained DNA substitutions. Neighbor-joining, maximum likelihood and maximum parsimony trees were constructed using PAUP 4.0b (Swofford, 1998), each with 1,000 bootstrap replications. Genetic distances among single spore cultures were computed in PAUP 4.0b (Swofford, 1998) using the TrN+I substitution model. The Mantel test was applied to verify the correlation between genetic and geographical distances using the software Arlequin 3.1 (Excoffier et al., 2005).

\section{Criteria to establish pairings between isolates}

Anastomosis between AMF isolates was evaluated in selected pairings between genetically different and genetically similar cultures. Six pairings were established to evaluate self anastomosis within and between germinating spores of the following isolates: WV310\#5, WV123A\#6, WV123A\#7, 1UnC\#7, 6AmA\#2 and CRwest\#8. Non-self anastomosis was evaluated in three pairings between genetically similar isolates (WV310\#5 x WV123A\#6, WV123A\#7 x WV123A\#6 and WV123A\#7 x WV310\#5) and seven pairings between genetically different isolates (WV310\#5 x 1UnC\#7, WV123A\#6 x 1UnC\#7, WV123A\#7 x 1UnC\#7, 6AmA\#2 x 1UnC\#7, 6AmA\#2 x CRwest\#8, 1UnC\#7 x CRwest\#8 and 1UnC\#5 x 1UnC\#7). 
Self and non-self anastomosis in symbiotic mycelia were evaluated using the same pairings described earlier. Six additional pairings were established to measure non-self anastomosis in a broader sampling of genetically similar isolates: 1UnC\#7 x CRwest\#8, CRcenter\#12 x WV101\#5, 6AmA\#2 x 1UnC\#5, Valley\#4 x Valley\#18, Valley\#4 x 6AmA\#3, Valley\#18 x 6AmA\#3. Another seven pairings were established to measure non-self anastomosis between a wider range of genetically different isolates: 1UnC\#5 x CRwest\#8, Valley\#4 x 1UnC\#7, Valley\#4 x CRwest\#8, Valley\#18 x 1UnC\#7, Valley\#18 x CRwest\#8, 6AmA\#3 x 1UnC\#7, 6AmA\#3 x CRwest\#8.

\section{Anastomosis in asymbiotic hyphae}

Spores were surface-sterilized (Bécard \& Fortin, 1988) and placed on cellophane membranes positioned into two-compartment, 10 -cm diameter Petri dishes. Two pieces of cellophane membranes were cut to fit the internal shape of each compartment. Membranes were placed on a thin layer of $\mathrm{M}$ medium, $\mathrm{pH}$ 6.0, lacking sucrose and vitamins (Bécard \& Fortin, 1988). To evaluate self anastomosis within a mycelium, one spore was placed in the center of each compartment using sterile reverse-action tweezers. To evaluate self and non-self anastomosis between paired mycelia, two spores were placed $3 \mathrm{~mm}$ apart in the center of each compartment (Croll et al., 2009). Fifteen replications were established for each single spore isolate and for each combination of paired spores.

Petri dishes were sealed with Parafilm and incubated at $28^{\circ} \mathrm{C}$ in the dark. After 14 days, germinated spores were observed for hyphal contacts using a Nikon Eclipse TE-2000S inverted microscope. For individual spores, all hyphal contacts were examined to detect any morphological changes. For paired spores, only the contacts between hyphae originating from different spores were examined. Compatible hyphal fusions were identified when interacting hyphae showed complete wall fusion and protoplasmic continuity (Giovannetti et al., 1999; $2001 ; 2004)$. The number of compatible hyphal fusions was divided by the total number of observed hyphal contacts in order to calculate percentage of anastomosis.

\section{Anastomosis in symbiotic hyphae}

A modified rhizohyphatron (Purin \& Morton, 2011) was used to monitor in situ hyphal interactions in the symbiotic phase of the AMF life cycle. Two versions of this system were 
used: (i) a single plant compartment to measure self anastomosis within a mycelium (Fig. 3a, b) and (ii) two plant compartments to measure self and non-self anastomosis between paired mycelia (Fig. 3c, d). Both versions consisted of plant compartments containing actively-growing mycorrhizal plants and a compartment housing three agar-coated microscope cover slips on which hyphae from plant compartment(s) could grow. All plastic components of the rhizohyphatron consisted of Schedule 40 PVC pipe fittings. The plant compartment was made using a $4.8 \mathrm{~cm}$ (inside diameter) $90^{\circ}$ PVC elbow. Two small holes were drilled in the base of the elbow for drainage. The cover slip compartment was a $4.0 \times 4.0 \mathrm{~cm}$ section of a PVC tube, within which plastic tabs to support cover slips were cut from $1.0 \times 7.5 \mathrm{~cm}$ pot labels and affixed to the wall of the PVC tube using white General Electric 100\% Silicon II sealant. One support $(1.0 \times 4.0 \mathrm{~cm})$ spanned the center of the tube and the other two supports $(1.0 \times 3.5 \mathrm{~cm})$ were spaced one $\mathrm{cm}$ above and below the center. Before assembly, all components were surfacesterilized by immersion in $10 \%$ bleach for 20 minutes followed by rinses with distilled water and $70 \%$ ethanol. Component parts then were exposed to ultraviolet light in a sterile hood for 20 minutes. At the time of assembly, the cover slip compartment remained empty. Each plant compartment was filled with premoistened, autoclaved Terragreen. Three 10-12-day-old sorghum seedlings were inoculated with approximately 200 spores by pipetting directly along the length of intertwined roots. Sorghum seedlings were transplanted into the Terragreen and the rhizohyphatrons were transferred to a growth room where they were maintained. Plants were watered ad libitum daily with double-distilled deionized water.

After mycorrhizal plants had grown for four weeks, rhizohyphatrons were transferred to a laminar hood to add cover slips. Cover slips (25 x 40mm, Ted Pella, Inc.) were coated with a thin layer of autoclaved $1.5 \%$ water agar containing $0.1 \mathrm{mg} \mathrm{kg}^{-1} \mathrm{P}$ (added as $\mathrm{KH}_{2} \mathrm{PO}_{4}$ ). To regulate thickness of the agar coating, $17 \mathrm{ml}$ of autoclaved agar was poured over 10 cover slips arranged on the bottom of a flat $8.8 \times 12.5 \mathrm{~cm}, 2 \mathrm{~cm}$ deep plastic tray. Once the agar solidified, cover slips were excised using a sterile dissecting blade and positioned in the compartment. In all units, care was taken to ensure that cover slips were in close contact with the nylon mesh so that hyphae from the plant compartment would migrate easily onto the agar surface of each cover slip. Rhizohyphatrons were returned to the growth room for ten days, at which time cover slips were removed from each unit for microscopic observation. 
Hyphae from paired plant compartments overlapped so that interactions were examined in detail in the central $30 \mathrm{~mm}$ region of each cover slip. Spores of G. clarum formed on all cover slips, thus confirming species identity. In all of the pairings made in this study, no fungal contaminants were observed. Contamination was eliminated by repeated cleaning of all adjacent surfaces with $70 \%$ ethanol and watering of plants only with double-distilled water.

All contacts between hyphae originating from opposing plant compartments were evaluated for morphological changes. Hyphal contacts were examined in three cover slips for each pairing (approximately 300 hyphal contacts per pairing). Hyphae from each contact point were tracked visually back to both ends of a cover slip to verify compartment of origin. Compatible hyphal fusions were identified and percentage of anastomosis was calculated as for germinating spores.

Percentages of self anastomosis were compared between single and paired spores using the t-Test. Spearman's rank correlation coeffient was calculated between the following combinations of variables: geographic distance + percentage of non-self anastomosis in germinating spores and genetic distance + percentage of non-self anastomosis in germinating spores. Analyses were performed using the software JMP version 5.0 (SAS Institute 1989-2002).

\section{Results}

\section{Genetic diversity of Glomus clarum at pasture and mining sites}

The 1014-bp concatenated sequences of two anonymous genetic markers Gcla69061F/Gcla690-553R and Gcla650-32F/Gcla650-552R (Table 2) used to genotype 17 isolates of Glomus clarum differentiated 15 distinct haplotypes. Isolates collected from Logan and Cassville pasture habitats showed little genetic differentiation despite a distance of almost $200 \mathrm{~km}$ between sites (mean pair-wise genetic distance of $0.002 \pm 0.002$ SD; Fig. 1, 2). In contrast, isolates from habitats disturbed by coal-mining were distinct genetically even though they all originated from sites less than $20 \mathrm{~km}$ apart (mean pair-wise genetic distance of $0.008 \pm 0.003 \mathrm{SD}$; Fig. 1, 2). The Mantel test revealed no significant correlation between geographic and genetic distance $(P=$ 0.678). 


\section{Anastomosis in asymbiotic mycelia}

Extensive asymbiotic hyphal networks emerging from germinating spores of G. clarum were formed in 14 days. The percentage of self anastomosis in a mycelium from a germinating spore ranged from 6 to $21 \%$. Self anastomosis occurred at higher frequencies in hyphae merging from two spores $(24.9 \% \pm 3.7 \% \mathrm{SE})$ than in hyphae growing from one spore $(12.6 \% \pm 3.7 \% \mathrm{SE})$ $(P<0.05$; Table 3). Non-self anastomosis was detected at low frequency in all pairings between genetically similar isolates from geographically close pasture sites (Table 4). Successful anastomosis was evident by complete wall fusion (Fig. 4a) and protoplasmic flow. When spores of genetically different isolates were paired, anastomosis was observed at an average frequency of 5.8\% (Table 4, Fig. 4b, c) only between isolates $1 \mathrm{UnC \# 5}$ and $1 \mathrm{UnC \# 7}$ from a mining site. Negative correlations were observed between genetic distance and percentage of non-self anastomosis (Spearman's rho $=-0.664 ; P=0.036$ ) and between geographic distance and percentage of non-self anastomosis (Spearman's rho $=-0.739 ; P=0.014$ ).

\section{Anastomosis in symbiotic mycelia}

Self anastomosis within a mycelium growing from a single plant compartment averaged $8.6 \% \pm 3.8 \%$ SE over all isolates (Table 3), with a range of 2-16\%. Self anastomosis between mycelia from two plant compartments was considerably higher, averaging $21.4 \% \pm 3.8 \% \mathrm{SE}$ over all isolates $(P<0.05$, Table 3$)$. All fusions were characterized by formation of bridges between paired hyphae (Fig. 5). Complete fusion between hyphae was confirmed by continuity in protoplasmic streaming. Non-self anastomosis was absent in all pairings, regardless of whether the paired isolates were genetically similar or different from each other (Table 4).

\section{Discussion}

In this study, clear distinctions were evident between asymbiotic and symbiotic phases of hyphal growth in G. clarum. Self anastomosis occurred in both asymbiotic and symbiotic hyphae, but non-self anastomosis was confined only to asymbiotic hyphae. Patterns of non-self interactions in symbiotic AMF mycelium, therefore, appear to be unique compared to other fungal groups. In Ascomycetes, hyphal interactions result in either fusion or rejection responses (Glass \& Kaneko, 2003). In AMF, neither response occurs once the fungus establishes symbiosis, so that inter-individual recognition is absent. 
The two phases of the AMF life cycle are easy to separate methodologically, thus providing the means to assess functional differences. Asymbiotic hyphae are examined exclusively in spore germination assays (Giovannetti et al., 1999; Croll et al., 2009). Symbiotic hyphae require living hosts, and so they were examined in a rhizohyphatron designed to observe interactions of hyphae traceable to mycorrhizal plants (Purin \& Morton, 2011).

Isolates of $G$. clarum could not be genotyped using primers that amplified microsatellite repeats in G. intraradices (Croll et al., 2008; Mathimaran et al., 2008), even though this species is in the same phylogenetic clade (Schwarzott et al., 2001; Silva et al., 2006). Analysis of the $G$. intraradices amplicons using these primers revealed that $46-90 \%$ nucleotide variation is in the microsatellite-flanking regions instead of in the repeat itself (Croll et al., 2008; Mathimaran et $a l .$, 2008). Even in ascomycete and basidiomycete fungi, variability in microsatellite-flanking regions is high (Guerin et al., 2004; Gauthier et al., 2007). Only two of ten products of flanking regions amplified by microsatellite primers were polymorphic in G. clarum. Similarly, only 18 of 80 primer pairs yielded polymorphic sequences in G. intraradices (Mathimaran et al., 2008).

Despite only two markers, distinct genotypes in the two single spore cultures from each of the nine sampling sites were differentiated. Only in the location WV123A were both isolates genetically identical. Differentiation observed in such small sample sizes indicates that any assemblage of spores collected from a location may contain more than one genotype. Therefore, studies using inocula from multiple spores are not likely to distinguish self from non-self interactions (Giovannetti et al., 2001; 2003).

Asymbiotic hyphal networks from germinating spores of G. clarum were extensively formed in 14 days instead of the 35 days reported for $G$. intraradices (Croll et al., 2009). An average of eight contacts was present per pairing of spores, compared to an average of one contact per paired spores of $G$. intraradices. Hyphal fusions might be of more limited benefit for initial "colony" formation in G. clarum than in other Glomus species. The overall frequency of self anastomosis in G. clarum (19\%) was lower than the 46-51\% measured in germinating spores of G. intraradices (Croll et al., 2009) and the 36-69\% measured in G. mosseae (Giovannetti et al., 1999).

Non-self anastomosis was observed between germinating spores of genetically similar and different isolates. Pairings of similar genotypes from neighboring locations showed a frequency of non-self anastomosis of less than 3\%. Even though the two markers were identical, 
it is unlikely that these fusions were self interactions because self-anastomosis averaged $25 \%$ (Table 3). Since similar frequencies of non-self anastomosis were measured between genetically different isolates of G. intraradices (Croll et al., 2009), the G. clarum non-self anastomoses likely occurred between isolates that differed in other loci of the genome.

Non-self anastomosis was observed only between isolates sampled from geographically close locations with similar environments. Likewise, G. intraradices was collected by Croll et al. (2009) from locations separated by less than 85 meters in the same field site. Non-self anastomosis in these two AMF species appears to occur only between isolates that are geographically close, suggesting that populations in similar environments are selecting for gene combinations that allow vegetative compatibility. Geographically distant isolates that also were genetically different failed to anastomose, as observed by Giovannetti et al. (2003) for $G$. mosseae. Non-self anastomosis between the genetically different isolates 1UnC\#5 and 1UnC\#7 collected from the same location provides further evidence to support this hypothesis.

Methods to study anastomosis in symbiotic hyphae have relied mainly on transformed root organ cultures (Bécard \& Fortin, 1988). Our approach was to use a rhizohyphatron which permitted symbiotic hyphae from plants to grow on an agar-coated cover slip (Purin \& Morton, 2011). The plant compartment from which a mycelium originated always could be identified because hyphae in the center of the cover slip were traceable back to a plant compartment.

Self anastomosis in symbiotic hyphal networks emerging from the same plant compartment averaged $8.6 \%$ across all isolates, which was only slightly higher than the $6.7 \%$ observed in situ from a genetically uncharacterized multi-spore culture of G. clarum (Purin \& Morton, 2011). In both experiments, self anastomosis was considerably lower than the $80 \%$ and $88 \%$ measured for symbiotic hyphae of $G$. proliferum and G. intraradices, respectively, growing from root organ cultures (Voets et al., 2006). This disparity may have a genetic basis, once frequencies of putative self anastomosis in paired symbiotic mycelia of $G$. mosseae range between 36 and 70\% (Giovannetti et al., 2004; Sbrana et al., 2010). Alternatively, it also may be caused by differences in the experimental environment.

Non-self anastomosis was never observed between symbiotic mycelia of any isolate of $G$. clarum, regardless of genetic identity. The lack of anastomosis was not an artifact of the experimental environment, because hyphal fusions were always observed when an isolate was paired with itself. The frequency of anastomosis was two-fold higher in hyphae from paired 
spores or plants than in hyphae originating from the same source. Germ tubes from some paired spores fused and formed a common hyphal network so fast that the zone of recognition could not be identified. Symbiotic hyphae from paired plants quickly established contact in the center region of the cover slip and produced more frequent bridges between long, thick runner hyphae rather than the fine hyphae on the periphery of each mycelium. These patterns indicate behavior of self recognition within a colony-forming network is distinct from that of hyphae meeting from separate colonies. Self anastomosis between hyphae originating from spatially separated plants has important implications for formation of common mycorrhizal networks (CMNs). Interconnection of roots and nutrient transfer between plants are possible through CMNs, which can affect population and community-level processes (Giovannetti et al., 2004; Simard \& Durall, 2004). Our data suggest that CMNs are formed only between hyphae originated from the same parental spore.

Hyphal fusions were typically characterized by bridges similar in structure to those reported in other studies (Giovannetti et al., 1999; 2001; Purin \& Morton, 2011). Although nuclear staining was not performed, perfect fusion was evidenced visually by continuity in cytoplasmic streaming. Non-recognition characterized the majority of hyphal contacts, with no evidence of post-fusion incompatibility reported by Croll et al. (2009) in G. intraradices or by Sbrana et al. (2010) in G. mosseae. Septation was observed in approximately $25 \%$ of the contacts between asymbiotic hyphae and in 5\% of the contacts between symbiotic hyphae. However, septa are observed in hyphal tips and certain regions of the mycelium during normal growth of AMF, and so presence of these structures may not reflect signs of incompatibility.

The occurrence of non-self anastomosis only in germinating spores may not be unusual. Some ascomycete fungi are able to anastomose only after a short period following conidia germination (Read et al., 2009). These species form specialized conidial anastomosis tubes (CATs) that allow fusion between vegetative incompatible individuals. The mechanism underlying fusion between CATs is unknown, but likely involves suppression of vic genes (Roca et al., 2005). Similar behavior may occur in AMF, where genes regulating vegetative interactions are differentially expressed in germinating spores. Certain genes involved in AMF metabolism have been reported to be differentially expressed in either asymbiotic versus symbiotic hyphae (Requena et al., 2003; Tamasloukht et al., 2003; Requena et al., 2007), and similar regulation may occur for genes involved in recognition. 
Results of this study and those from Croll et al. (2009) are complementary. The frequency of self anastomosis in paired spores averaged $25 \%$ in $G$. clarum and $48 \%$ in $G$. intraradices. In non-self interactions, the frequency of anastomosis decreased in both species to less than $10 \%$. Anastomosis behavior in other glomeromycotan genera has not been studied in detail, but likely differs from Glomus. For example, self anastomosis in symbiotic hyphae of representative Gigaspora and Scutellospora species growing from root organ cultures is less than 10\% (de la Providencia et al., 2005; Voets et al., 2006) and completely absent when growing from plants in a rhizohyphatron (Purin \& Morton, 2011). In Paraglomus and Ambispora as well, no self anastomosis is observed in symbiotic mycelia (Purin \& Morton, 2011). With rare self anastomosis, non-self anastomosis likely will be even rarer in these lineages. All of the available evidence indicates that non-self anastomosis is a relatively rare phenomenon in glomeromycotan fungi, occurring mostly or possibly even exclusively during the asymbiotic phase of fungal growth and development. However, even the low frequencies of non-self fusions in asymbiotic hyphal networks of both G. clarum and G. intraradices still is sufficient to permit horizontal gene transfer and produce new genotypes in a population (Croll et al., 2009).

\section{Acknowledgements}

The authors wish to thank Teresa Pawlowska for insightful guidance and Jianbo Yao for the use of the inverted microscope in his laboratory. Rhizohyphatrons were built and maintained with the help of Bill Wheeler and Robert Bills. We thank David Douds for the DC2 isolate of $R i$ T-DNA transformed carrot roots developed by Guillaume Bécard. Funding was provided by a CAPES/Fulbright Ph.D. scholarship to Sonia Purin and NSF grant DEB-0649341 to Joseph Morton. 
Table 1. Microsatellite repeats used as PCR primers to amplify flanking regions in genomic DNA extracted from a pooled population of Glomus clarum spores.

\begin{tabular}{llcc}
\hline \multicolumn{1}{c}{ Repeat } & \multicolumn{1}{c}{ Reference } & $\begin{array}{c}\text { TA } \\
\left({ }^{\mathbf{C}} \mathbf{C}\right)\end{array}$ & $\begin{array}{c}\text { Approximate amplicon size } \\
(\mathbf{b p})\end{array}$ \\
\hline$(\mathrm{CA})_{9} *$ & Longato \& Bonfante $(1997)$ & 45 & - \\
$(\mathrm{GTG})_{5}$ & Longato \& Bonfante $(1997)$ & 52 & 500,600 \\
$(\mathrm{GACA})_{5} *$ & Longato \& Bonfante $(1997)$ & 48 & 400,650 \\
$(\mathrm{AAT})_{8}$ & Douhan \& Rizzo $(2003)$ & 37 & - \\
$(\mathrm{AAC})_{8}$ & Douhan \& Rizzo $(2003)$ & 55 & 650 \\
$(\mathrm{AGC})_{5} *$ & Douhan \& Rizzo $(2003)$ & 50 & 550,650 \\
$(\mathrm{CAT})_{8}$ & Douhan \& Rizzo $(2003)$ & 55 & - \\
$(\mathrm{CCG})_{5} *$ & Douhan \& Rizzo $(2003)$ & 58 & - \\
$(\mathrm{CAA})_{6}$ & Mathimaran et al. $(2008)$ & 43 & - \\
$(\mathrm{TAT})_{8} *$ & Mathimaran et al. $(2008)$ & 36 & - \\
$(\mathrm{TTA})_{8}$ & Mathimaran et al. $(2008)$ & 37 & $400,600,690,770,1155$ \\
$(\mathrm{AATG})_{5} *$ & Mathimaran et al. $(2008)$ & 43 & - \\
$(\mathrm{AATGGT})_{5}^{*}$ & Mathimaran et al. $(2008)$ & 55 & \\
\hline
\end{tabular}

* The number of repeats was modified in order to increase the annealing temperature. 
Table 2. Primer pairs used to amplify microsatellite-flanking regions from single spore cultures of Glomus clarum. Primers were designed from ten amplicons generated by microsatelliteprimers (Table 1).

\begin{tabular}{|c|c|c|c|c|}
\hline $\begin{array}{c}\text { Origin of sequence: } \\
\text { microsatellite - } \\
\text { amplicon size (bp) }\end{array}$ & Primer name & Primer sequence (5'-3') & $\begin{array}{r}\text { TA } \\
\left({ }^{\circ} \mathrm{C}\right)\end{array}$ & $\begin{array}{l}\text { Amplicon } \\
\text { size (bp) }\end{array}$ \\
\hline \multirow[t]{2}{*}{$(\mathrm{GACA})_{5}-400$} & Gcla400-9F & GACAGACAGACAATACCAACCA & 55 & 300 \\
\hline & Gcla400-308R & ACTGCTGAATTTCACGTGCTC & & \\
\hline \multirow{2}{*}{$(\mathrm{GACA})_{5}-650$} & Gcla650-32F & AGCCCATCCGTGTCTTT & 55 & 521 \\
\hline & Gcla650-552R & GCATTCATTCGAATCTGTTGG & & \\
\hline \multirow[t]{2}{*}{$(\mathrm{AAC})_{8}-650$} & Gcla650-125F & TCATGTAATTCATAATACCTTCCAAT & 55 & 448 \\
\hline & Gcla650-572R & CGAAGAGAACGATAGTCATGGA & & \\
\hline \multirow[t]{2}{*}{$(\mathrm{AGC})_{5}-550$} & Gcla550-80F & GTATCGGCGGCATCAAAC & 57 & 418 \\
\hline & Gcla550-497R & TAGCCGATAGCGGAAACG & & \\
\hline \multirow[t]{2}{*}{$(\mathrm{AGC})_{5}-650$} & Gcla650-77F & ACCGTCACTGCCACATACG & 56 & 555 \\
\hline & Gcla650-631R & GCAGCAGCGATAGTTGGAA & & \\
\hline \multirow[t]{2}{*}{$(\mathrm{AATG})_{5}-400$} & Gcla400-87F & CCСCTTAAGATGAACGGAATC & 56 & 311 \\
\hline & Gcla400-397R & TGAATGAATGAAATTAGAGGCTACC & & \\
\hline \multirow[t]{2}{*}{$(\mathrm{AATG})_{5}-600$} & Gcla600-52F & TTTCCTTGGTCATCATGTCG & 57 & 502 \\
\hline & Gcla600-553R & TTTCCATTCATCATCCCAAC & & \\
\hline \multirow[t]{2}{*}{$(\mathrm{AATG})_{5}-690$} & Gcla690-61F & CGACGCTATTCCCATCA & 57 & 493 \\
\hline & Gcla690-553R & CACCGCCAAATTGAACCT & & \\
\hline \multirow[t]{2}{*}{$(\mathrm{AATG})_{5}-770$} & Gcla770-99F & ATCCGTCATCTCCGAACTTT & 57 & 575 \\
\hline & Gcla770-673R & GGCCGAATTTGTGACGTTAT & & \\
\hline \multirow[t]{2}{*}{$(\mathrm{AATG})_{5}-1155$} & Gcla1155-98F & AAAATGGCCAATTGGAACC & 57 & 961 \\
\hline & Gcla1155-1058R & TCACCGTCCAATCAAGCA & & \\
\hline
\end{tabular}


Table 3. Percentage of hyphal contacts that resulted in compatible hyphal fusion (self anastomosis) in relation to the total number of hyphal contacts observed. Fusions were observed in germinating spores (asymbiotic phase) and in extraradical mycelium of Glomus clarum growing from mycorrhizal plants (symbiotic phase).

\begin{tabular}{ccccc}
\hline \multirow{2}{*}{ Isolate } & Single spore & Paired spores & Single plant & Paired plants \\
\hline 1UnC\#7 & 6.3 & 8.0 & 15.6 & 37.4 \\
6AmA\#2 & 6.6 & 19.7 & 10.3 & 24.5 \\
CRwest\#8 & 17.4 & 37.5 & 11.7 & 32.4 \\
WV123A\#6 & 13.4 & 30.3 & 2.4 & 5.4 \\
WV123A\#7 & 11.4 & 18.1 & 4.7 & 11.1 \\
WV310\#5 & 20.6 & 35.7 & 6.8 & 17.8 \\
Average \pm SE & $12.6 \pm 3.7 \mathrm{~b}$ & $24.9 \pm 3.7 \mathrm{a}$ & $8.6 \pm 3.8 \mathrm{~b}$ & $21.4 \pm 3.8 \mathrm{a}$ \\
\hline
\end{tabular}


Table 4. Genetic distance, geographic distance and percentage of contacts that resulted in compatible hyphal fusion (non-self anastomosis) in relation to the total number of hyphal contacts observed. Data from pairings where non-self anastomosis was evaluated in germinating spores (asymbiotic phase) and extraradical mycelium of Glomus clarum growing from mycorrhizal plants (symbiotic phase).

\begin{tabular}{|c|c|c|c|c|}
\hline $\begin{array}{c}\text { Pairing } \\
\text { of } \\
\text { isolates }\end{array}$ & $\begin{array}{l}\text { Genetic } \\
\text { distance } \\
(\mathrm{Tr} N+\mathrm{I})\end{array}$ & $\begin{array}{c}\text { Geographic } \\
\text { distance } \\
\text { (meters) }\end{array}$ & $\begin{array}{c}\text { Anastomosis in } \\
\text { asymbiotic } \\
\text { phase }(\%)\end{array}$ & $\begin{array}{c}\text { Anastomosis in } \\
\text { symbiotic phase } \\
(\%)\end{array}$ \\
\hline 6AmA\#2 x 1UnC\#7 & 0.013 & 15 & 0 & 0 \\
\hline 6AmA\#2 x CRwest\#8 & 0.011 & 11000 & 0 & 0 \\
\hline 1UnC\#7 x CRwest\#8 & 0.008 & 11000 & 0 & 0 \\
\hline $1 \mathrm{UnC \# 5} \times 1 \mathrm{UnC \# 7}$ & 0.011 & 0 & 5.8 & 0 \\
\hline 1UnC\#7 x WV310\#5 & 0.014 & 190000 & 0 & 0 \\
\hline 1UnC\#7 x WV123A\#6 & 0.014 & 190000 & 0 & 0 \\
\hline 1UnC\#7 x WV123A\#7 & 0.014 & 190000 & 0 & 0 \\
\hline WV310\#5 x WV123A\#6 & 0.000 & 20 & 0.9 & 0 \\
\hline WV123A\#7 x WV123A\#6 & 0.000 & 0 & 1.6 & 0 \\
\hline WV123A\#7 x WV310\#5 & 0.000 & 20 & 2.2 & 0 \\
\hline
\end{tabular}




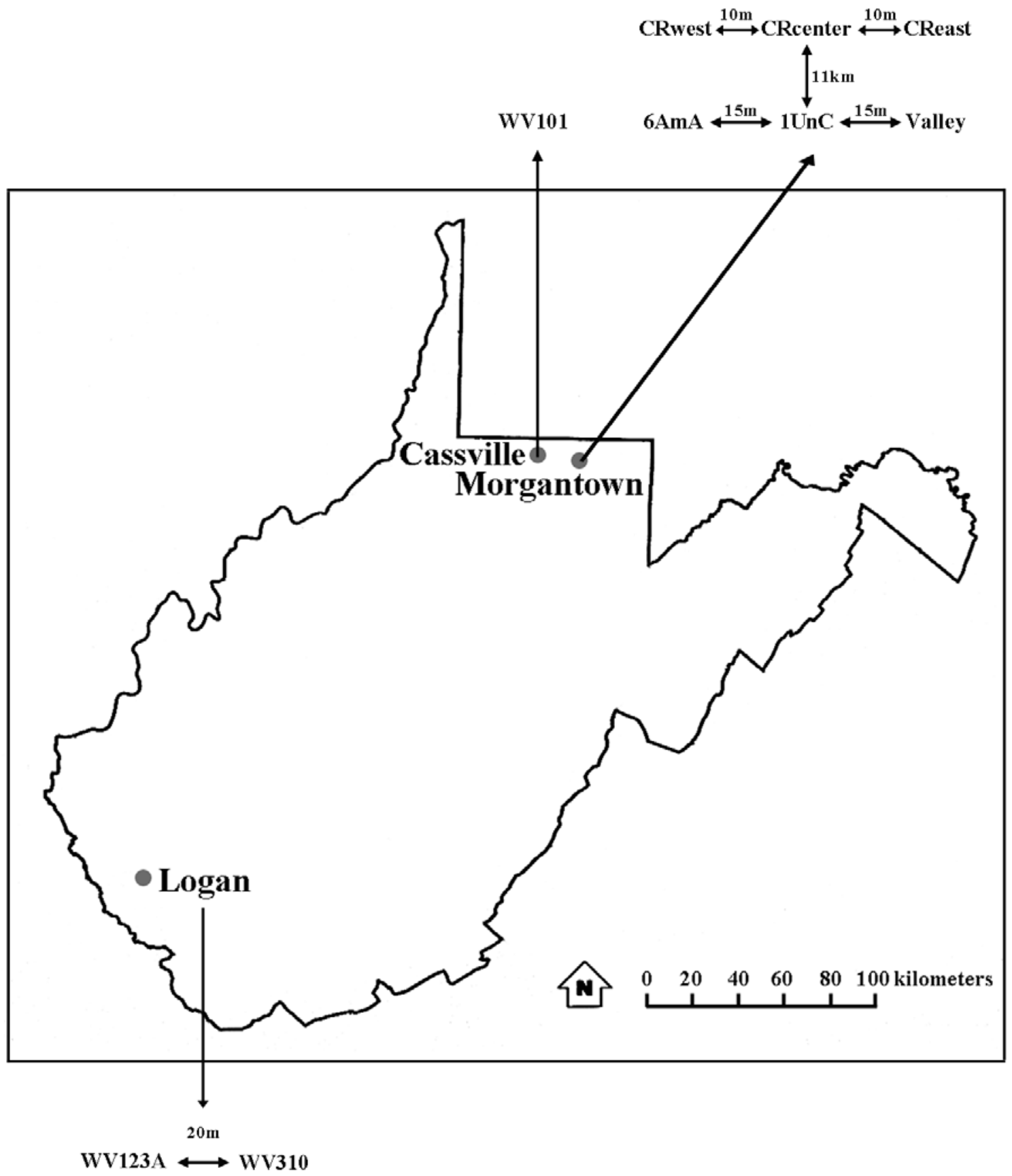

Figure 1. Map of West Virginia state (USA) indicating sampled locations and respective distances. 6AmA, Valley, 1UnC, CRwest, CRcenter and CReast are abandoned coal stripmine sites near Morgantown. WV101 originated from a pasture near Cassville. WV123A and WV310 originated from sites in 4-6 year-old hydroseeded pasture mixture near Logan, WV. Two single spore cultures were obtained from each sampling population (except CReast, with one culture). 


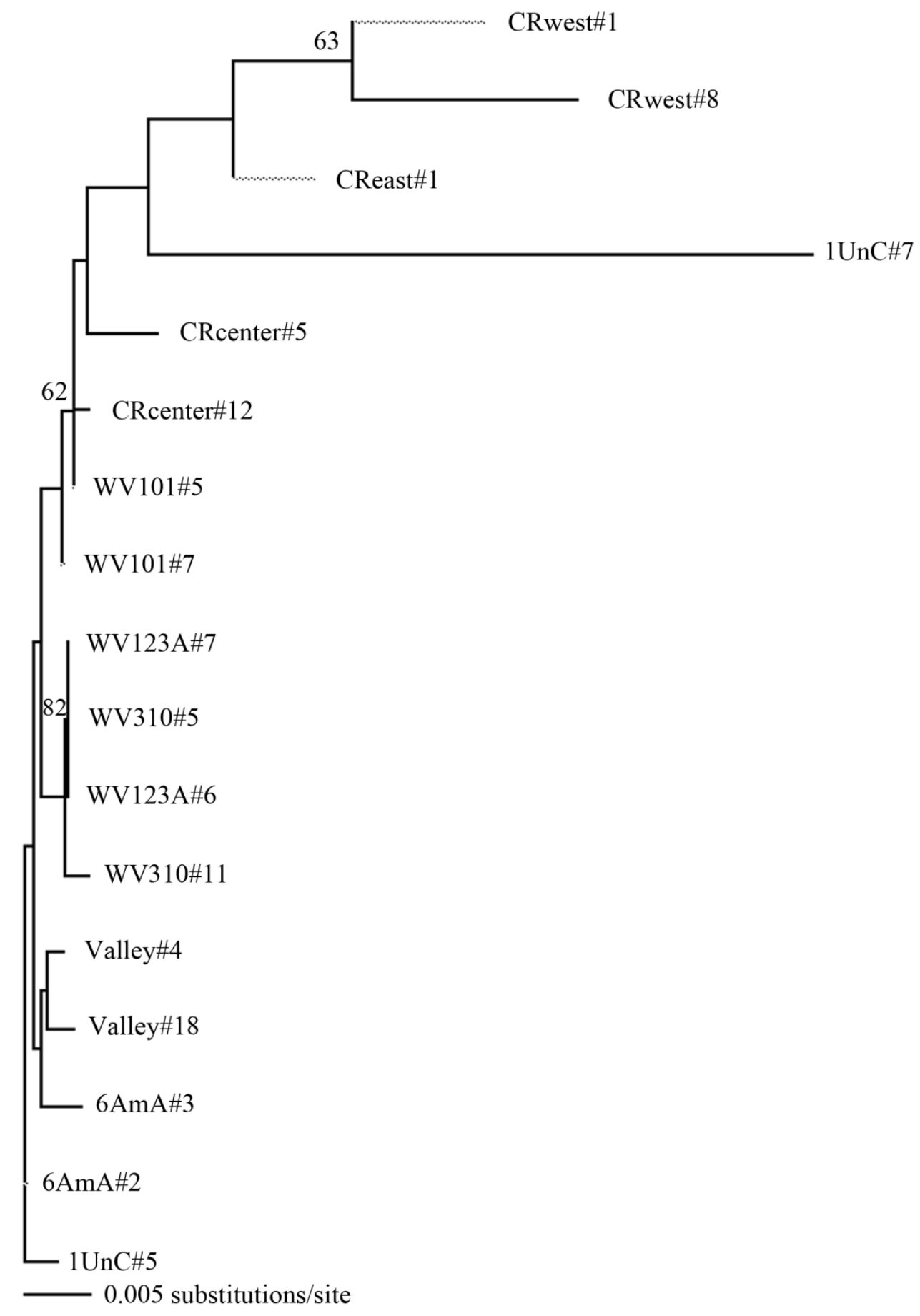

Figure 2. Neighbor-joining tree reconstructed from two concatenated sequences of microsatellite-flanking regions amplified from the G. clarum genome. Numbers at nodes indicate bootstrap values $\geq 50 \%$. The model of nucleotide substitution is $\operatorname{TrN}+\mathrm{I}$, with a rate substitution matrix of $A$ to $\mathrm{G}$ at 14.34 and $\mathrm{C}$ to $\mathrm{T}$ at 6.25 and proportion of invariable sites of 0.97 . 


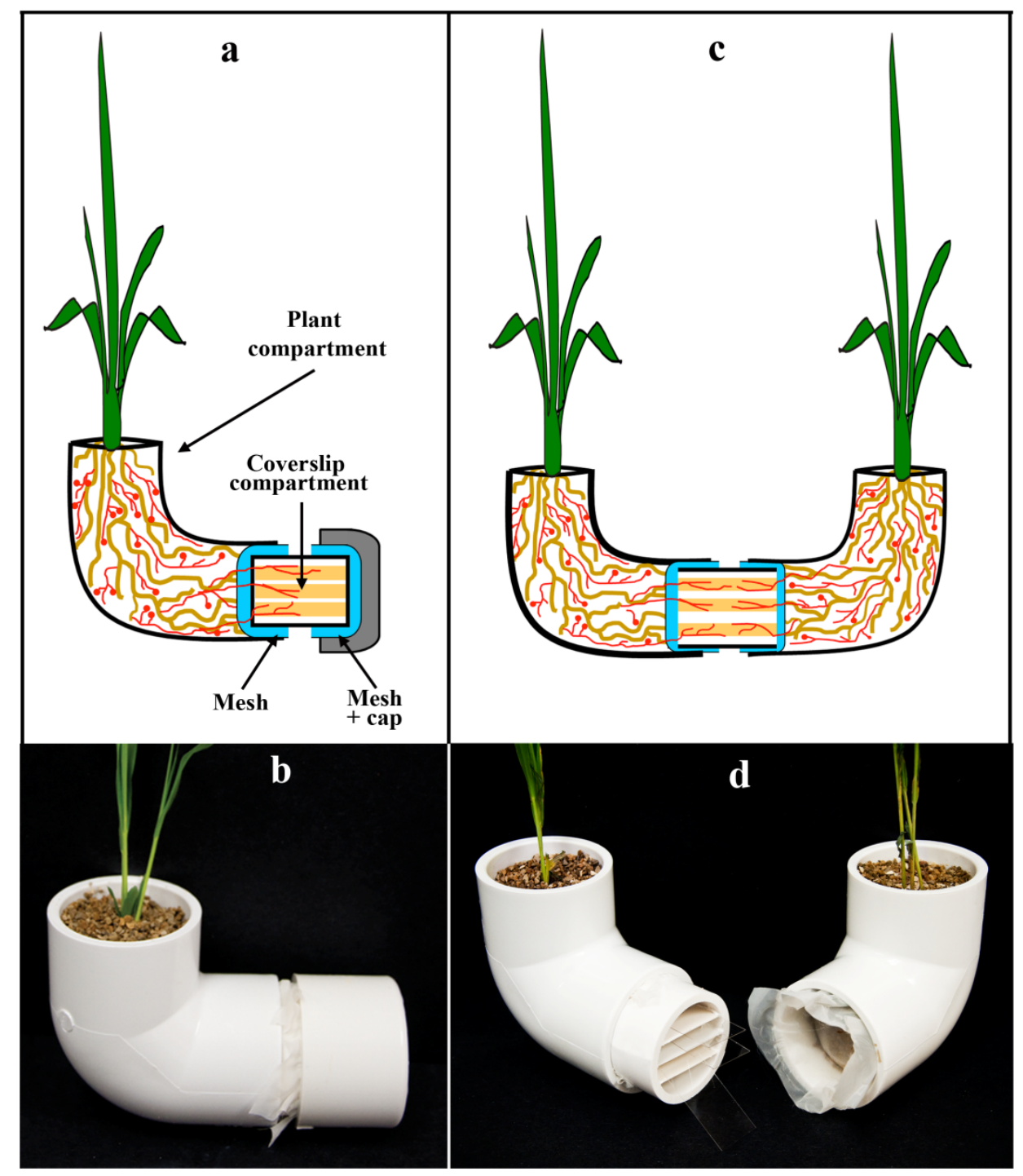

Figure 3. Plant-fungal growth system (rhizohyphatron) designed to study anastomosis in isolates of Glomus clarum. a) Illustration of a single plant compartment unit. The cover slip compartment provides placement for three agar-coated cover slips. Nylon mesh with $41 \mu \mathrm{m}$ openings (Sefar America Inc., Depew, NY) separates contents of the plant growth compartment from the cover slip compartment. Nylon mesh + cap tightly seal open end of the cover slip compartment. b) Assembled single unit containing sorghum seedlings preinoculated with spores. c) Illustration of a two plant compartment unit. Nylon mesh (blue lines) blocks roots so that only hyphae grow onto agar-coated cover slips in the cover slip compartment. d) Open rhizohyphatron showing mesh barrier, plastic supports for agar-coated cover slips spaced 1-cm apart in the center compartment, and sorghum seedlings preinoculated with spores. 

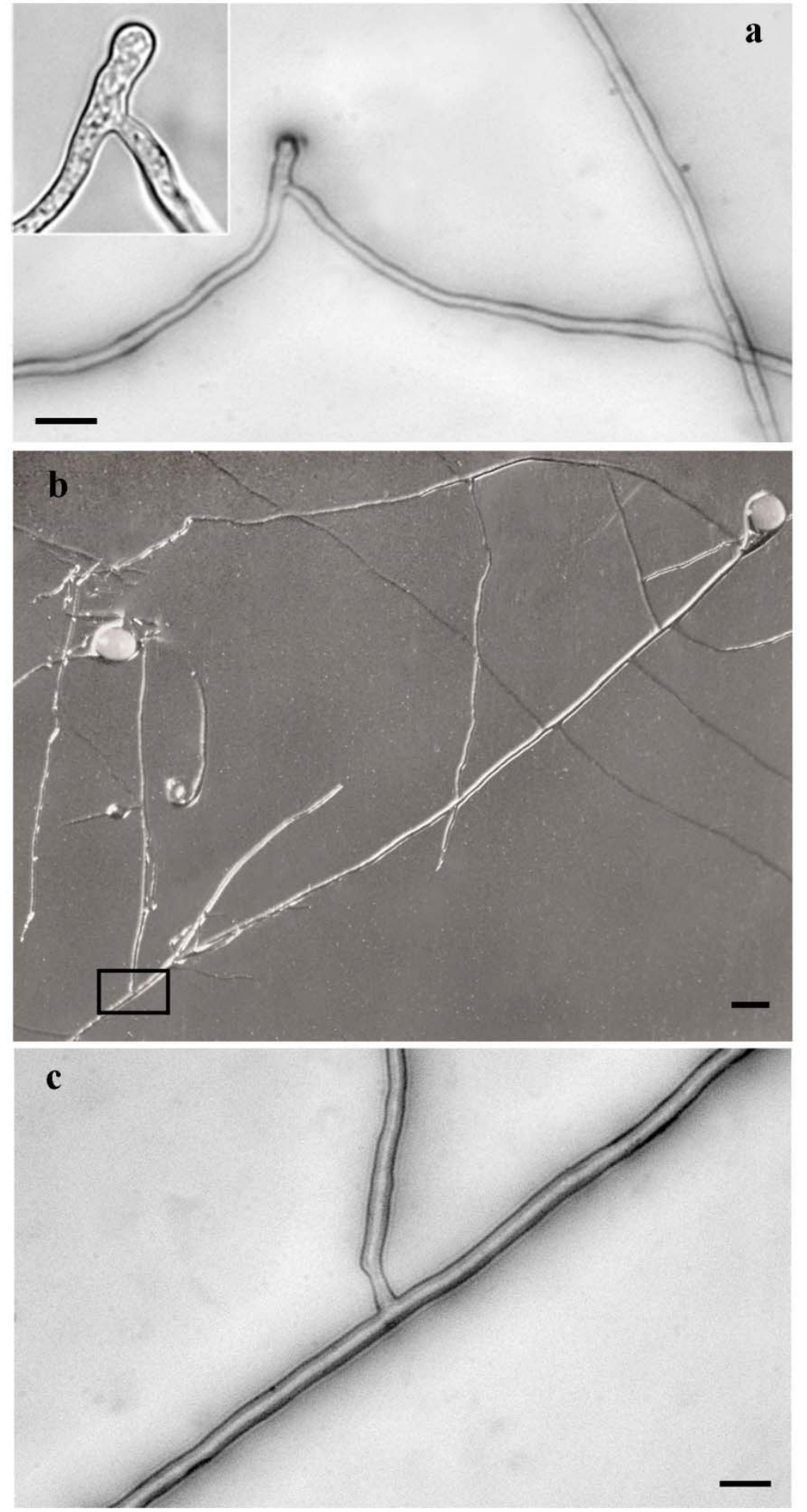

Figure 4. Non-self anastomosis between asymbiotic hyphae of Glomus clarum. a) Fusion between hyphae of isolates WV123A\#6 and WV123A\#7, with insert showing complete wall fusion and protoplasm continuity, bar $=25 \mu \mathrm{m}$. b) Paired spores of the isolates 1UnC\#5 and

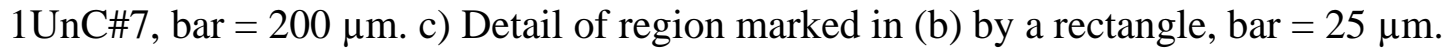



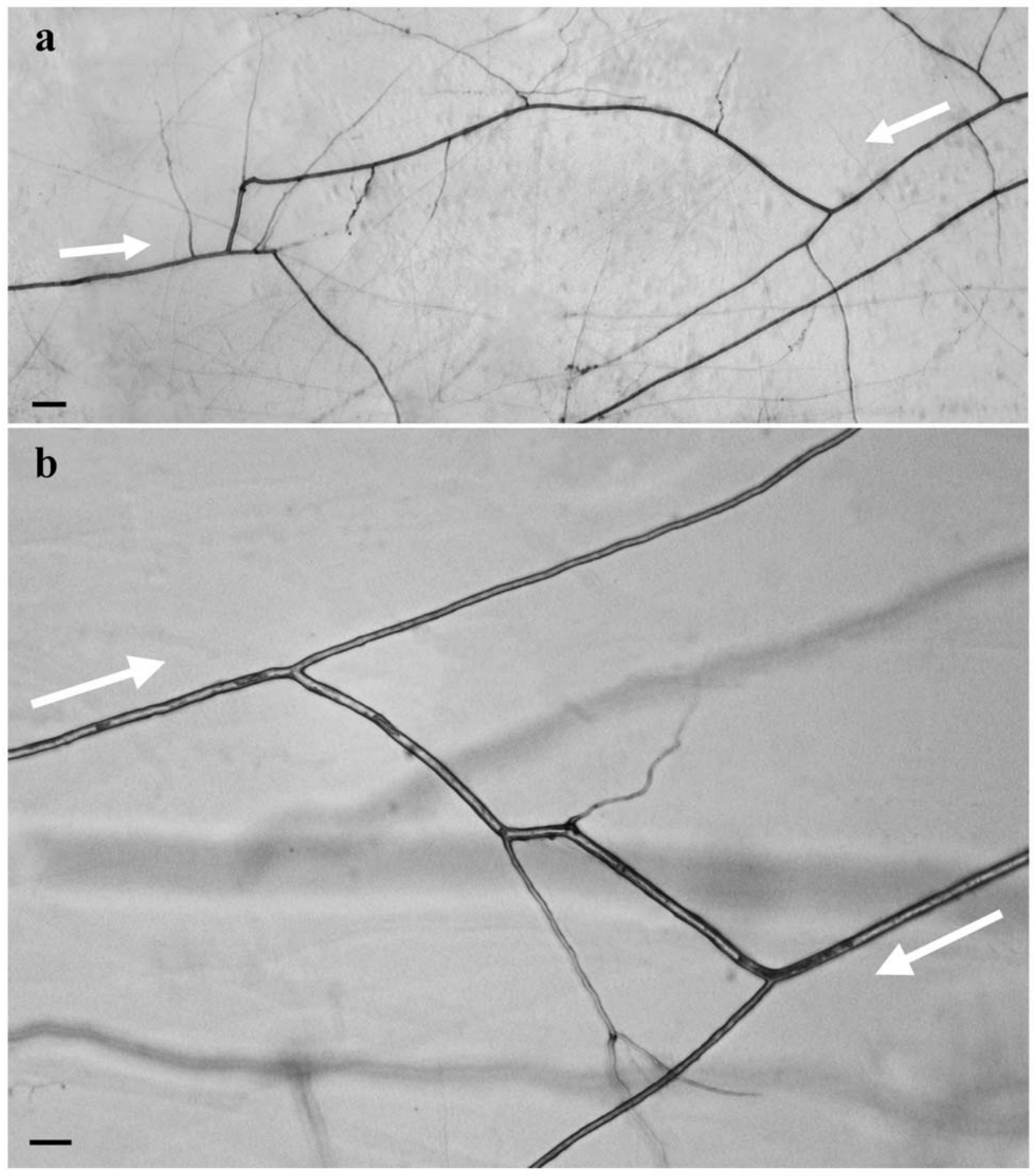

Figure 5. Self anastomosis between symbiotic hyphae of Glomus clarum from paired plant compartments. a) A long bridge between two hyphae of isolate 1UnC\#7 from opposite compartments (arrows), bar $=100 \mu \mathrm{m}$. b) Another long bridge from isolate $1 \mathrm{UnC \# 7}$ forming between two hyphae from opposite compartments passing each other (arrows). Cytoplasmic flow into the bridge is evident, bar $=50 \mu \mathrm{m}$. 


\section{References}

Aanen DK, Debets AJM, de Visser JAGM, Hoekstra RF. 2008. The social evolution of somatic fusion. BioEssays 30: 1193-1203.

Altschul SF, Gish W, Miller W, Myers EW, Lipman DJ. 1990. Basic local alignment search tool. Journal of Molecular Biology 215: 403-10.

Bécard G, Fortin JA. 1988. Early events of vesicular arbuscular mycorrhiza formation on Ri TDNA transformed roots. New Phytologist 108: 211-218.

Bécard G, Piché Y. 1990. Physiological factors determining vesicular-arbuscular mycorrhizal formation in host and non-host Ri TDNA transformed roots. Canadian Journal of Botany 68: 1260-1264.

Bever JD, Wang M. 2005. Arbuscular mycorrhizal fungi - Hyphal fusion and multigenomic structure. Nature 433: E3-E4.

Cardenas Flores A, Draye X, Bivort C, Cranenbrouck S, Declerck S. 2010. Impact of multispores in vitro subcultivation of Glomus sp. MUCL 43194 (DAOM 197198) on vegetative compatibility and genetic diversity detected by AFLP. Mycorrhiza 20: 415-425.

Caten CE, Jinks JL. 1966. Heterokaryosis: its significance in wild homothallic ascomycetes and fungi imperfecti. Transactions of the British Mycological Society 49: 81-93.

Cortesi P, Milgroom MG. 1998. Genetics of vegetative incompatibility in Cryphonectria parasitica. Applied and Environmental Microbiology 64: 2988-2994.

Croll D, Giovannetti M, Koch AM, Sbrana C, Ehinger M, Lammers PJ, Sanders IR. 2009. Nonself vegetative fusion and genetic exchange in the arbuscular mycorrhizal fungus Glomus intraradices. New Phytologist 181: 924-937.

Croll D, Wille L, Gamper HA, Mathimaran N, Lammers PJ, Corradi N, Sanders IR. 2008. Genetic diversity and host plant preferences revealed by simple sequence repeat and mitochondrial markers in a population of the arbuscular mycorrhizal fungus Glomus intraradices. New Phytologist 178: 672-687.

de la Providencia IE, de Souza FA, Fernandez F, Delmas NS, Declerck S. 2005. Arbuscular mycorrhizal fungi reveal distinct patterns of anastomosis formation and hyphal healing mechanisms between different phylogenic groups. New Phytologist 165: 261-271.

de Souza FA, Declerck S. 2003. Mycelium development and architecture, and spore production of Scutellospora reticulata in monoxenic culture with Ri T-DNA transformed carrot roots. Mycologia 95: 1004-1012.

Debets AJM, Griffiths AJF. 1998. Polymorphism of het-genes prevents resource plundering in Neurospora crassa. Mycological Research 102: 1343-1349. 
Douhan GW, Rizzo DM. 2003. Amplified fragment length microsatellites (AFLM) might be used to develop microsatellite markers in organisms with limited amounts of DNA applied to arbuscular mycorrhizal (AM) fungi. Mycologia 95: 368-373.

Excoffier L, Laval G, Schneider S. 2005. Arlequin ver. 3.0: An integrated software package for population genetics data analysis. Evolutionary Bioinformatics Online 1: 47-50.

Friese CF, Allen MF. 1991. The spread of VA mycorrhizal fungal hyphae in the soil: inoculum types and external hyphal architecture. Mycologia 83: 409-418.

Gauthier N, Dalleau-Clouet C, Fargues J, Bon M-C. 2007. Microsatellite variability in the entomopathogenic fungus Paecilomyces fumosoroseus: genetic diversity and population structure. Mycologia 99: 693-704.

Gerdemann JW, Nicolson TH. 1963. Spores of mycorrhizal Endogone species extracted from soil by wet sieving and decanting. Transactions of the British Mycological Society 46: 235-244.

Giovannetti M, Azzolini D, Citernesi AS. 1999. Anastomosis formation and nuclear and protoplasmic exchange in arbuscular mycorrhizal fungi. Applied and Environmental Microbiology 65: 5571-5575.

Giovannetti M, Fortuna P, Citernesi AS, Morini S, Nuti MP. 2001. The occurrence of anastomosis formation and nuclear exchange in intact arbuscular mycorrhizal networks. New Phytologist 151: 717-724.

Giovannetti M, Sbrana C, Avio L, Strani P. 2004. Patterns of below-ground plant interconnections established by means of arbuscular mycorrhizal networks. New Phytologist 164: 175-181.

Giovannetti M, Sbrana C, Strani P, Agnolucci M, Rinaudo V, Avio L. 2003. Genetic diversity of isolates of Glomus mosseae from different geographic areas detected by vegetative compatibility testing and biochemical and molecular analysis. Applied and Environmental Microbiology 69: 616-624.

Glass NL, Kaneko I. 2003. Fatal attraction: non-self recognition and heterokaryon incompatibility in filamentous fungi. Eukaryotic Cell 2: 1-8.

Glass NL, Kuldau GA. 1992. Mating type and vegetative incompatibility in filamentous Ascomycetes. Annual Review of Phytopathology 30: 201-224.

Glass NL, Rasmussen C, Roca MG, Read ND. 2004. Hyphal homing, fusion and mycelial interconnectedness. TRENDS in Microbiology 12: 135-141.

Gregory PH. 1984. The fungal mycelium: an historical-perspective. Transactions of the British Mycological Society 82: 1-11. 
Guerin F, Franck P, Loiseau A, Devaux M, Le Cam B. 2004. Isolation of 21 new polymorphic microsatellite loci in the phytopathogenic fungus Venturia inaequalis. Molecular Ecology Notes 4: $268-270$.

Hall TA. 1999. BioEdit: a user-friendly biological sequence alignment editor and analysis program for Windows 95/98/NT. Nucleic Acids Symposium Series 41: 95-98.

Jinks JL. 1952. Heterokaryosis: a system of adaptation in wild fungi. Proceedings of the Royal Society of London 140: 83-99.

SAS Institute. 1989-2002. JMP Version 5.0. SAS Institue. Cary, North Carolina, USA.

Li XL, George E, Marschner H. 1991. Extension of the phosphorus depletion zone in VAmycorrhizal white clover in a calcareous soil. Plant and Soil 136: 41-48.

Liu YC, Milgroom MG. 1996. Correlation between hypovirus transmission and the number of vegetative incompatibility (vic) genes different among isolates from a natural population of Cryphonectria parasitica. Phytopathology 86: 79-86.

Longato S, Bonfante P. 1997. Molecular identification of mycorrhizal fungi by direct amplification of microsatellite regions. Mycological Research 101: 425-432.

Mathimaran N, Falquet L, Ineichen K, Picard C, Redecker D, Boller T, Wiemken A. 2008. Microsatellites for disentangling underground networks: Strain-specific identification of Glomus intraradices, an arbuscular mycorrhizal fungus. Fungal Genetics and Biology 45: 812-817.

Posada D, Crandall KA. 1998. Modeltest: testing the model of DNA substitution. Bioinformatics 14: 817-818.

Purin S, Morton JB. 2011. In situ analysis of anastomosis in representative genera of arbuscular mycorrhizal fungi. Mycorrhiza. doi: 10.1007/s00572-010-0356-9.

Read ND, Lichius A, Shoji J, Goryachev AB. 2009. Self-signalling and self-fusion in filamentous fungi. Current Opinion in Microbiology 12: 608-615.

Requena N, Breuninger M, Franken P, Ocón A. 2003. Symbiotic status, phosphate, and sucrose regulate the expression of two plasma membrane $\mathrm{H}^{+}$-ATPase genes from the mycorrhizal fungus Glomus mosseae. Plant Physiology 132: 1540-1549.

Requena N, Serrano E, Ocón A, Breuninger M. 2007. Plant signals and fungal perception during arbuscular mycorrhiza establishment. Phytochemistry 68: 33-40.

Roca MG, Read ND, Wheals AE. 2005. Conidial anastomosis tubes in filamentous fungi. FEMS Microbiology Letters 249: 191-198. 
Rozen S, Skaletsky HJ. 2000. Primer3 on the WWW for general users and for biologist programmers. In: Krawetz S, Misener S, eds. Bioinformatics Methods and Protocols: Methods in Molecular Biology. Totowa, NJ: Humana Press, 365-386.

Sbrana D, Fortuna P, Giovannetti M. 2010. Plugging into the network: belowground connections between germlings and extraradical mycelium of arbuscular mycorrhizal fungi. Mycologia. doi:10.3852/10-125.

Schüßler A, Schwarzott D, Walker C. 2001. A new fungal phylum, the Glomeromycota: phylogeny and evolution. Mycological Research 105: 1413-1421.

Schwarzott D, Walker C, Schüßler A. 2001. Glomus, the largest genus of the arbuscular mycorrhizal fungi (Glomales), is nonmonophyletic. Molecular Phylogenetics and Evolution 21: 190-197.

Silva GA, Lumini E, Maia LC, Bonfante P, Bianciotto V. 2006. Phylogenetic analysis of Glomeromycota by partial LSU rDNA sequences. Mycorrhiza 16: 183-189.

Simard SW, Durall DM. 2004. Mycorrhizal networks: a review of their extent, function, and importance. Canadian Journal of Botany 82: 1140-1165.

Smith SE, Dickson S, Smith FA. 2001. Nutrient transfer in arbuscular mycorrhizas: how are fungal and plant processes integrated? Australian Journal of Plant Physiology 28: 683-694.

Swofford DL. 1998. PAUP* 4.0 Beta Version. Sinauer, Sunderland, MA.

Tamasloukht M, Séjalon-Delmas N, Kluever A, Jauneau A, Roux C, Bécard G, Franken P. 2003. Root factors induce mitochondrial-related gene expression and fungal respiration during the developmental switch from asymbiosis to presymbiosis in the arbuscular mycorrhizal fungus Gigaspora rosea. Plant Physiology 131: 1468-1478.

Tamura K, Nei M. 1993. Estimation of the number of nucleotide substitutions in the control region of mitochondrial DNA in humans and chimpanzees. Molecular Biology and Evolution 10: $512-526$.

Thompson JD, Gibson TJ, Plewniak F, Jeanmougin F, Higgins DG. 1997. The CLUSTAL_X windows interface: flexible strategies for multiple sequence alignment aided by quality analysis tools. Nucleic Acids Research 25: 4876-4882.

Voets L, de la Providencia IE, Declerck S. 2006. Glomeraceae and Gigasporaceae differ in their ability to form hyphal networks. New Phytologist 172: 185-188. 


\section{CONCLUSIONS}

The rhizohyphatron invented in this study is an experimental break-through because it permits the study of anastomosis in any culturable AMF species. This methodology allows a shift of focus from germinating spores to symbiotic hyphae, and both life cycle phases can be studied together. The rhizohyphatron can be used also as a comparative tool to study structure and behavior of mycelium in diverse AMF lineages.

Anastomosis mediates development of symbiotic hyphal networks in plants colonized by populations of Glomus species. Other taxonomic groups (Ambispora, Gigaspora, Paraglomus, Scutellospora) are unique in that symbiotic hyphae do not form linked networks via anastomosis when populations are used as source of inoculum. Such distinct behavior between Glomus and other AMF groups may indicate other unique symbiotic interactions/traits.

Self anastomosis occurs in both asymbiotic hyphae (germinating spores) and in symbiotic hyphae (mycorrhizae) of G. clarum. Non-self anastomosis only occurs in the asymbiotic phase at a low rate, so only during this short period is gene flow possible. Anastomosis of symbiotic

hyphae from different plants can only occur when genotypes are identical (self anastomosis). The absence of non-self anastomosis in symbiotic hyphae likely extends to all other AMF lineages, since self anastomosis between paired colonies is absent in other groups such as Gigaspora and Scutellospora.

Distinct processes of non-self recognition in asymbiotic vs. symbiotic phases suggest that other physiological processes also may be unique to each phase of the life cycle and should be investigated. AMF seem to be unique amongst fungal groups because inter-individual interactions are limited to germinating spores. Once in an association, each individual grows and functions in isolation. 


\section{APPENDIX}

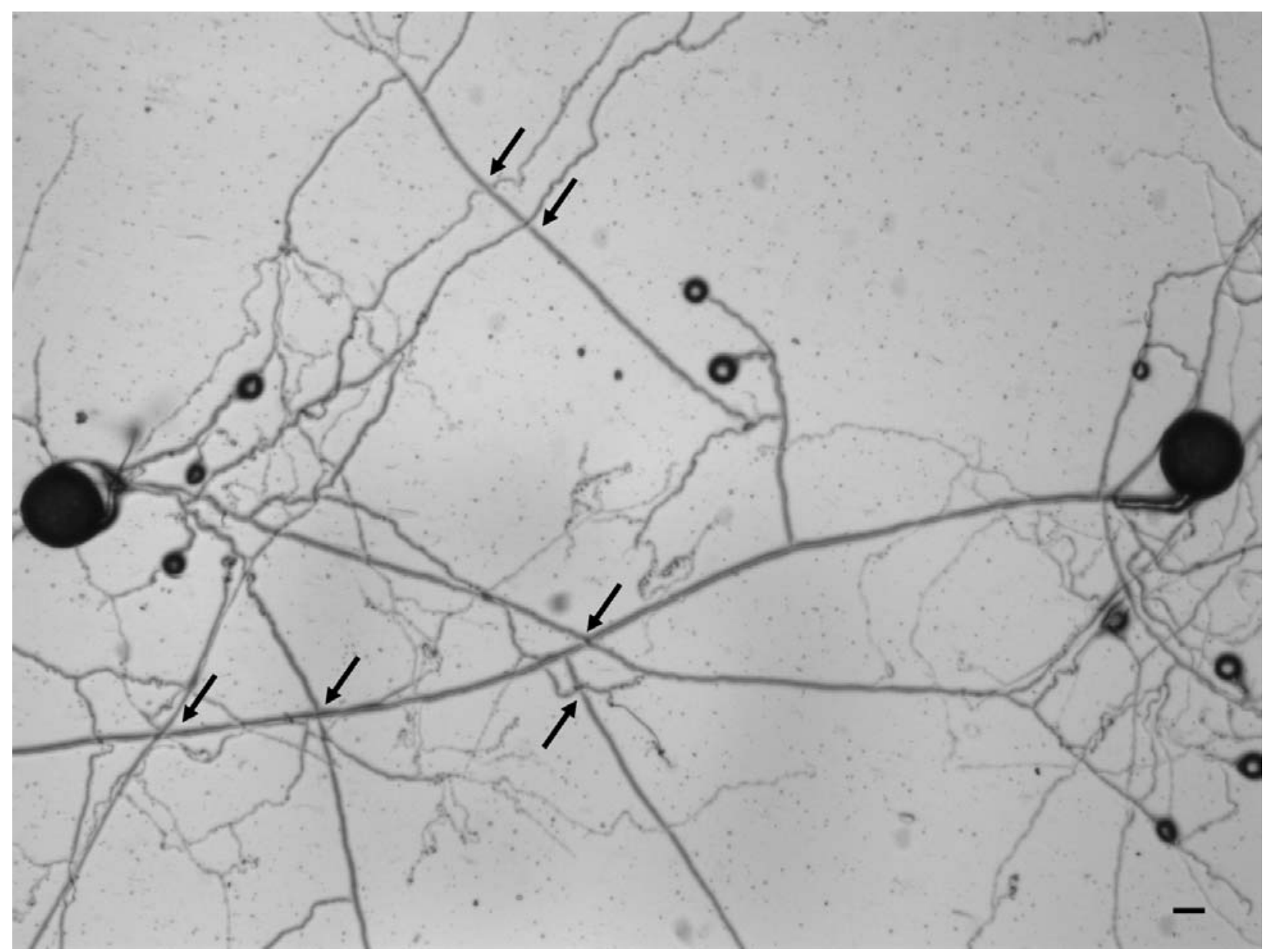

Figure 1. Germinating spores of Glomus clarum isolates WV123A\#6 (left) and 1UnC\#7 (right) on a cellophane membrane over $\mathrm{M}$ medium. Note hyphal contacts (arrows) in regions where hyphae overlap. Bar $=100 \mu \mathrm{m}$. 


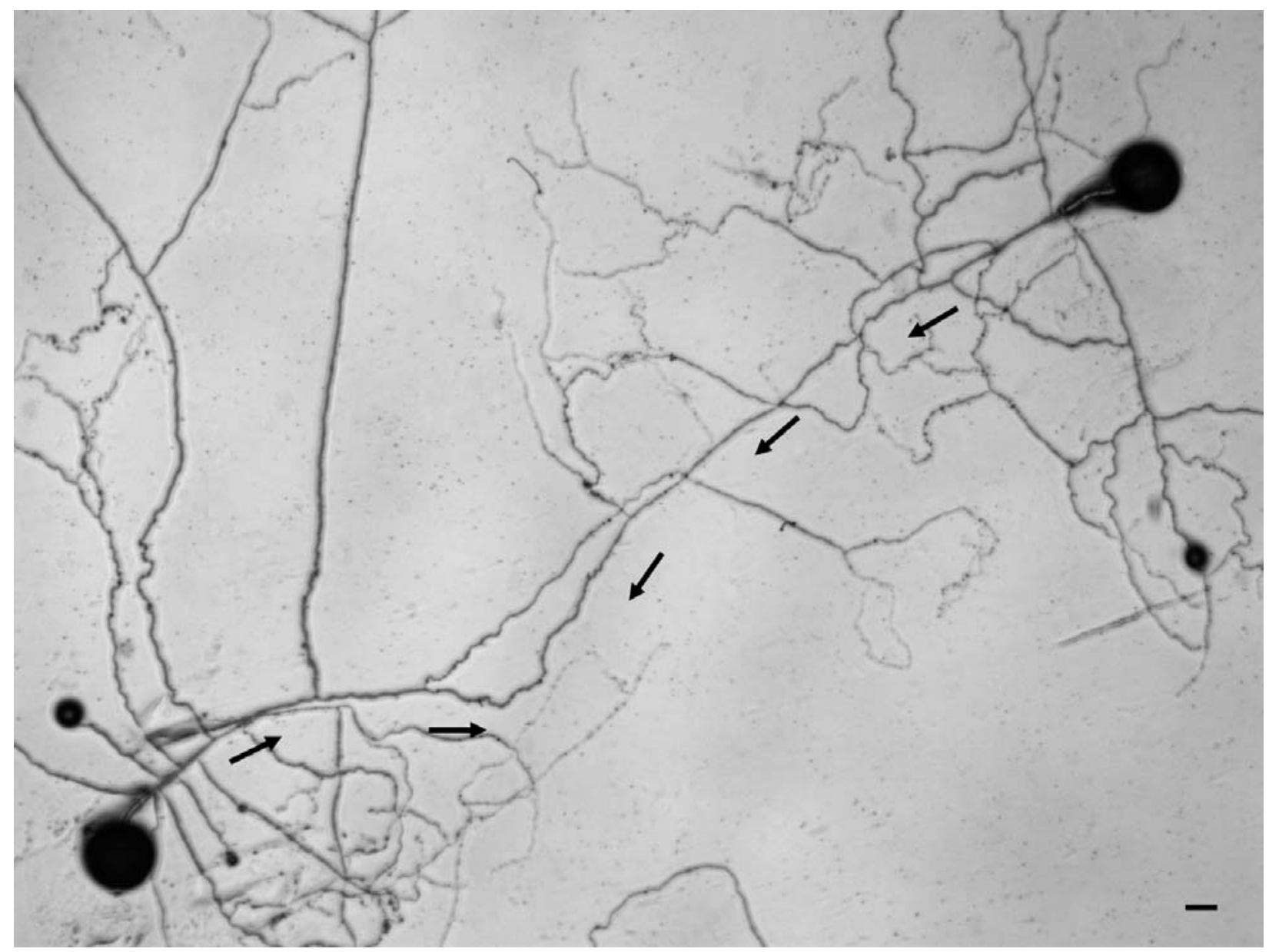

Figure 2. Germinating spores of the Glomus clarum isolates WV123A\#6 on a cellophane membrane over M medium. The main hypha from each spore fused to form one continuous hypha. Bar $=100 \mu \mathrm{m}$. 


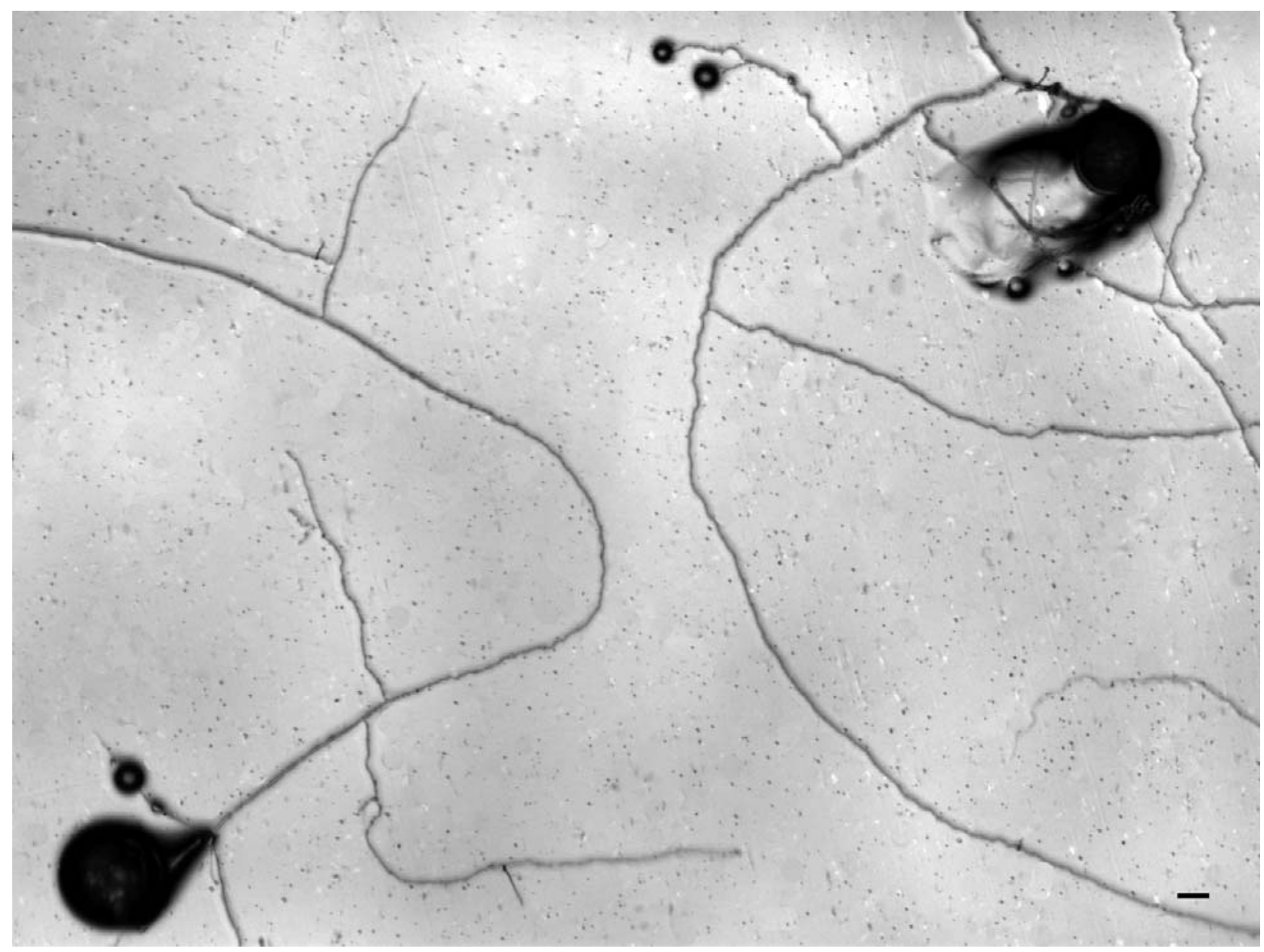

Figure 3. Germinating spores of Glomus clarum isolates 6AmA\#2 (left) and 1UnC\#7 (right) on a cellophane membrane over M medium. The main hyphae from each spore are directed away from each other and thereby avoid any physical contact. Bar $=100 \mu \mathrm{m}$. 


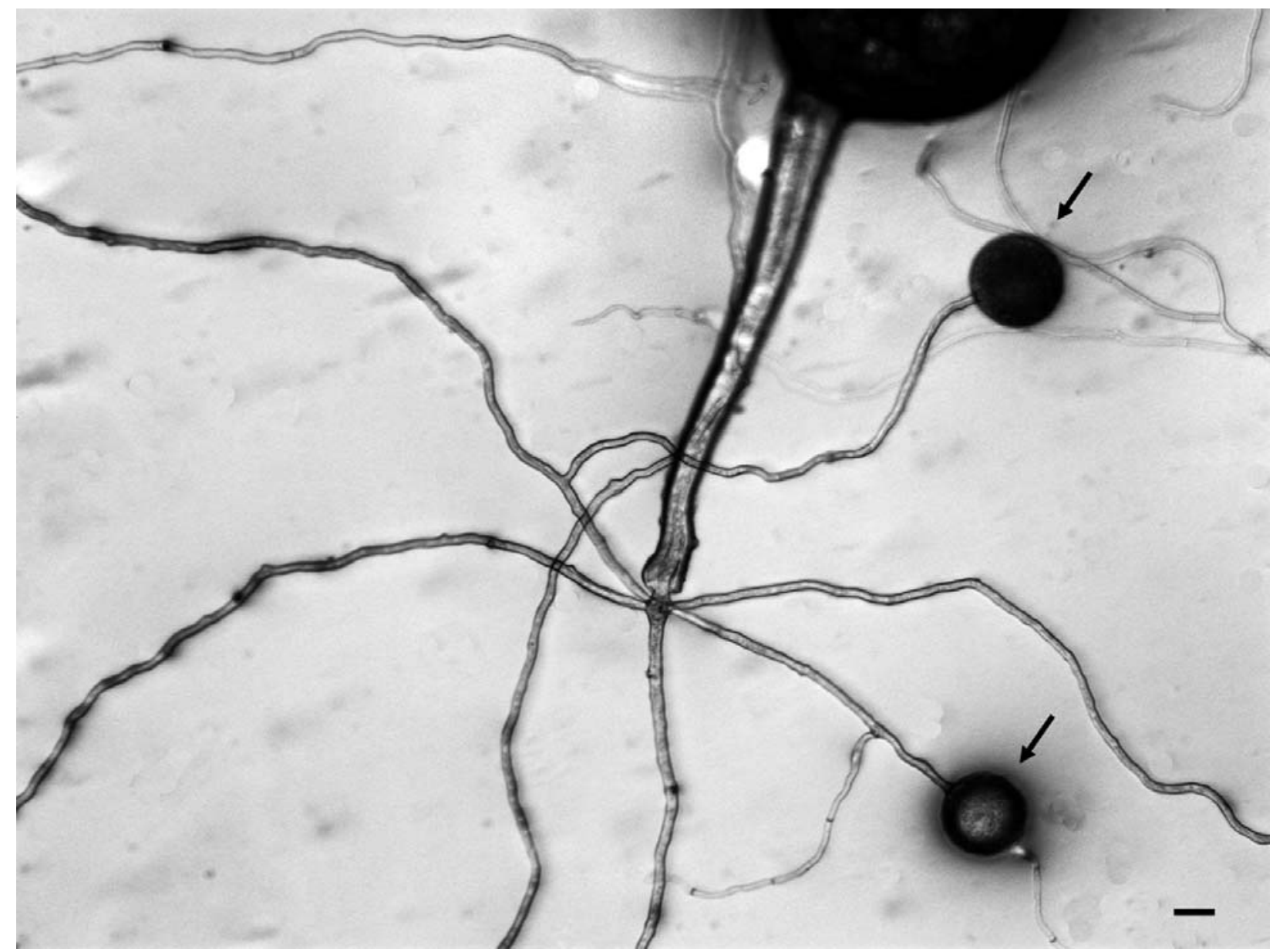

Figure 4. Germinating spore of Glomus clarum isolate WV123A\#7 on a cellophane membrane over M medium. The main hypha branches immediately after emergence from the broken end of the subtending hypha. Two "spore-like" structures (arrows) are formed 14 days after germination. Bar $=25 \mu \mathrm{m}$. 


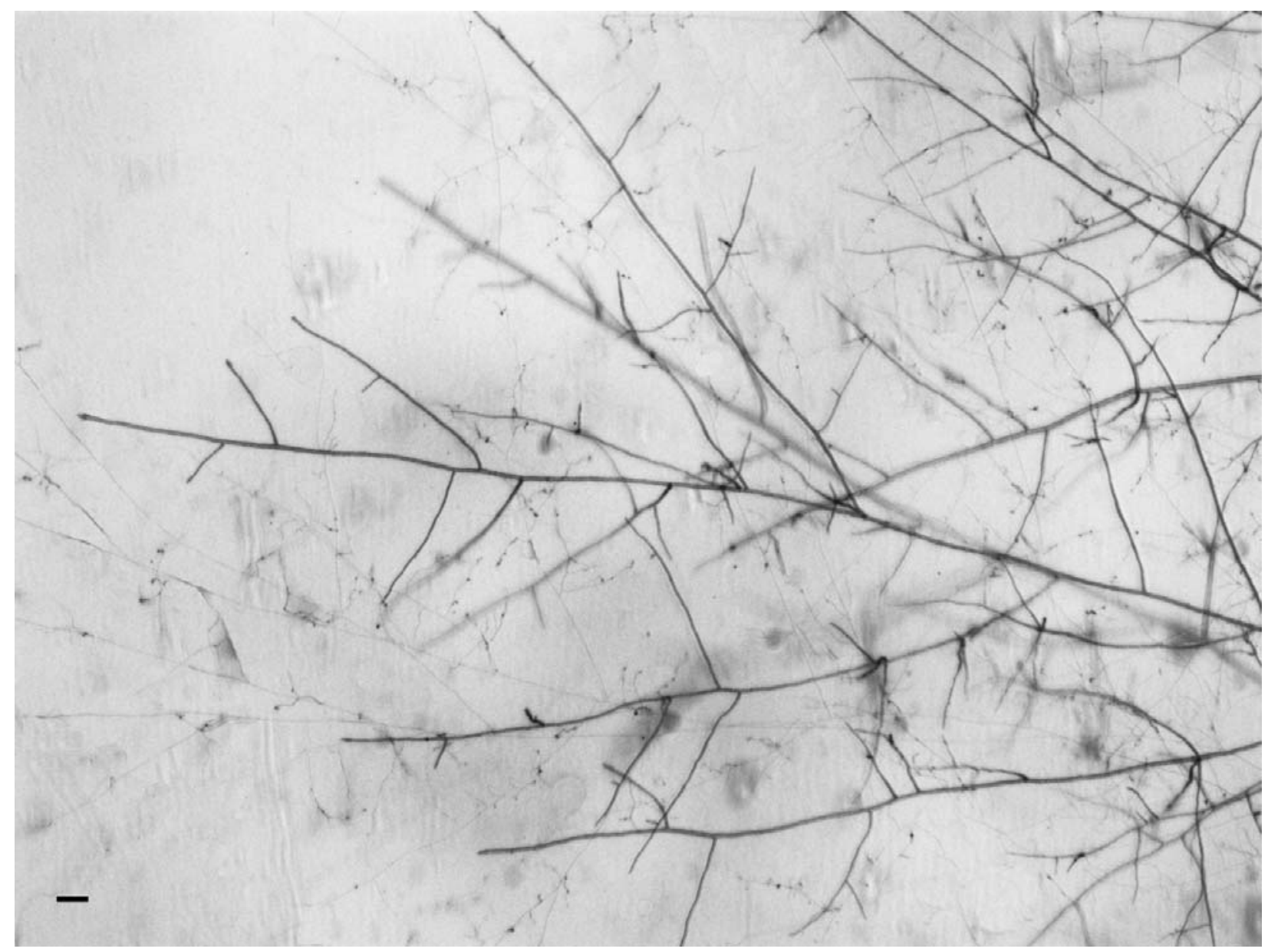

Figure 5. Symbiotic mycelium of Glomus clarum isolate CRwest\#8 growing on a cover slip coated with $1.5 \%$ agar. Bar $=100 \mu \mathrm{m}$. 


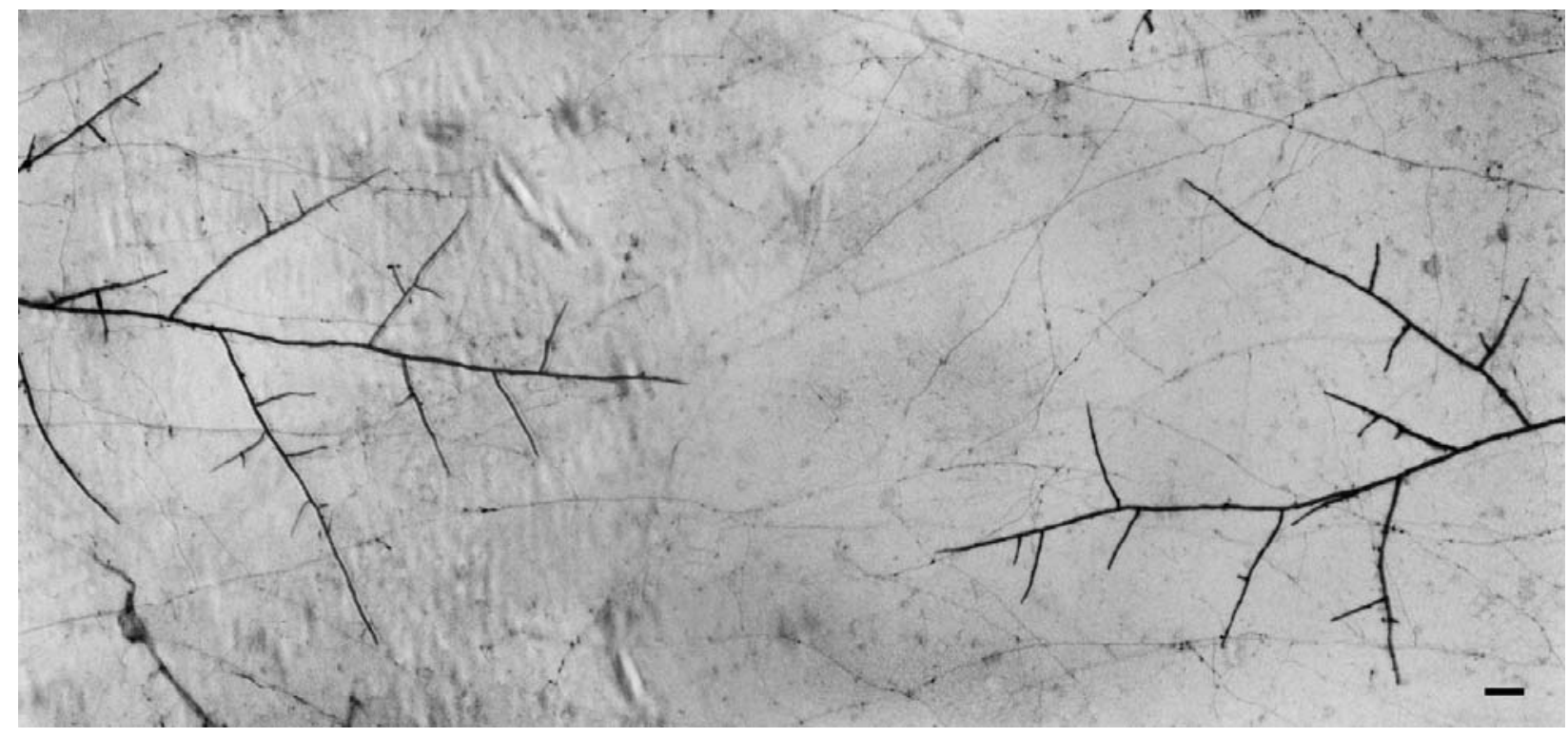

Figure 6. Symbiotic mycelia of Glomus clarum isolates $1 \mathrm{UnC \# 7} \mathrm{(left)} \mathrm{6AmA \# 3} \mathrm{(right)} \mathrm{growing}$ on a cover slip coated with $1.5 \%$ agar. Hyphae from each isolate originated from mycorrhizal plant compartments in direct contact with the cover slip. Bar $=100 \mu \mathrm{m}$. 


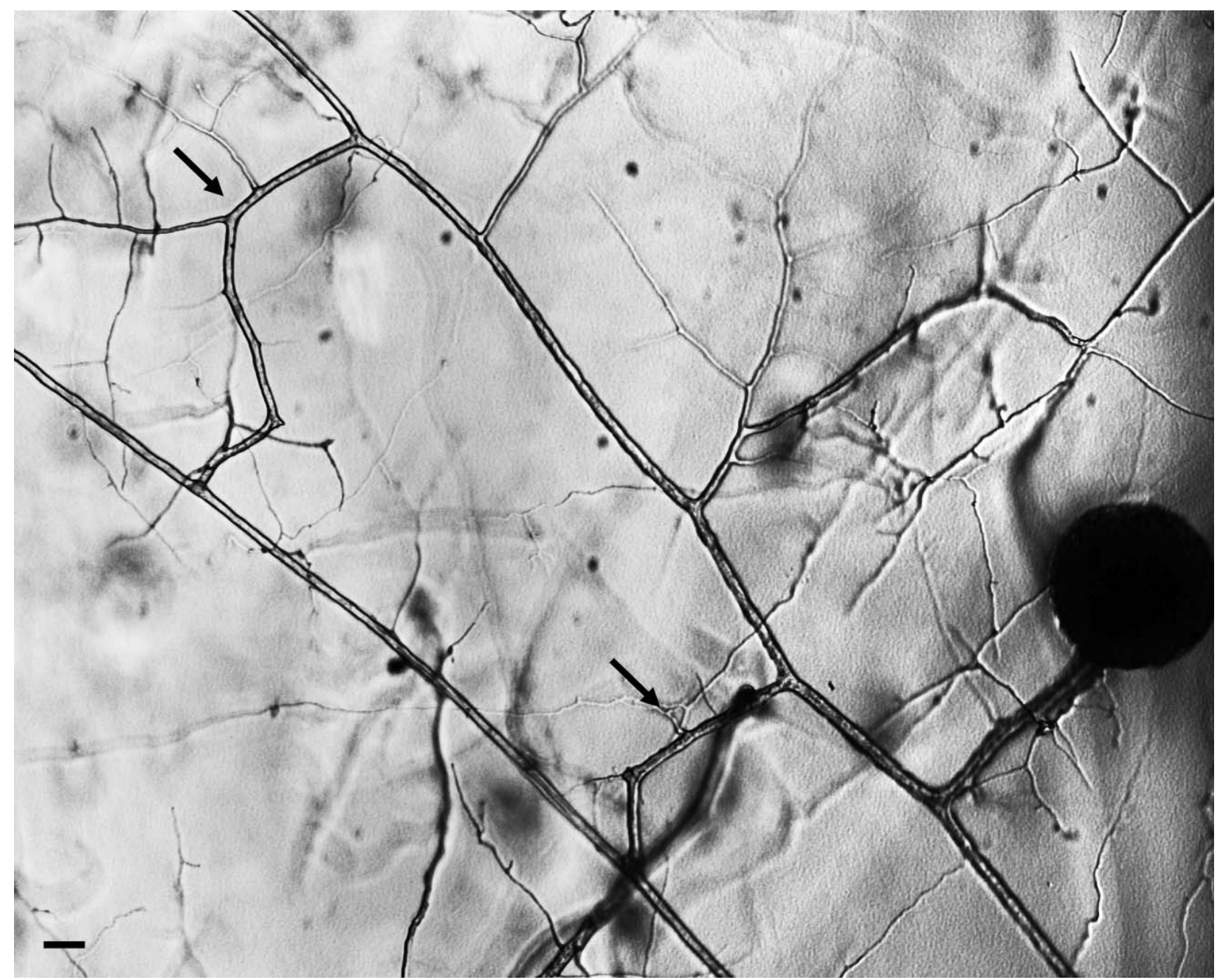

Figure 7. Self anastomosis in symbiotic mycelium of Glomus clarum isolate 1UnC\#7 growing on a cover slip coated with $1.5 \%$ agar. Two long bridges are formed (arrows) together with one spore. $\operatorname{Bar}=50 \mu \mathrm{m}$. 


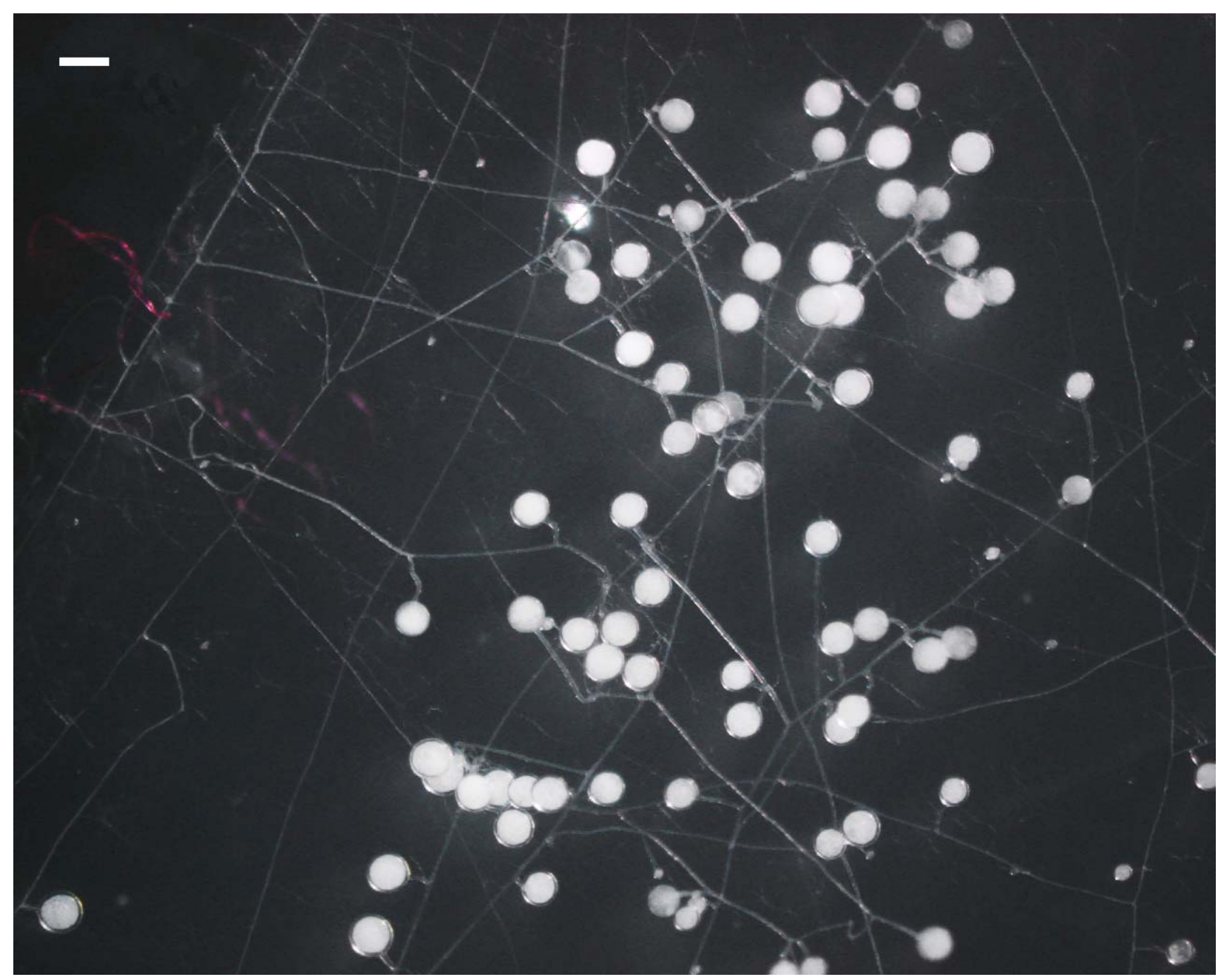

Figure 8. Symbiotic mycelium of Glomus clarum isolate 6AmA\#2 growing on a cover slip coated with $1.5 \%$ agar. Intense sporulation occurs 6 weeks after inoculation of sorghum seedlings. Bar $=200 \mu \mathrm{m}$. 


\section{CURRICULUM VITAE}

\section{Sonia Purin}

Rua 15 de novembro, 611 Bairro Carijós, Indaial SC 89130-000 Brasil

spurinwvu@gmail.com

\section{Education}

Ph.D. in Agricultural Sciences, with emphasis on Applied and Environmental Microbiology, Spring 2011 (expected), sponsored by Fulbright and CAPES

West Virginia University, Morgantown, WV, USA

M.Sc. in Soil Sciences, 2005

Universidade do Estado de Santa Catarina, Lages, SC, Brasil

B.S. in Biological Sciences, 2002

Universidade Regional de Blumenau, Blumenau, SC, Brasil

\section{Research Experience}

Research Assistantship, 2010 - 2011

West Virginia University, Morgantown, WV - Dr. Joseph Morton laboratory.

Maintenance of the INVAM culture collection and preparation of material supplied to other researchers. Establishment of in vitro root organ cultures of several isolates of arbuscular mycorrhizal fungi. Other responsibilities included ordering laboratory supplies, training and monitoring visitors in the laboratory.

\section{Professional development}

Workshop on Active Learning Techniques for the science, technology, engineering, and mathematics (STEM) disciplines

Office of Graduate Education and Life, West Virginia University, Morgantown, WV, 2010

\section{Publications}

\section{Refereed Journal Articles}

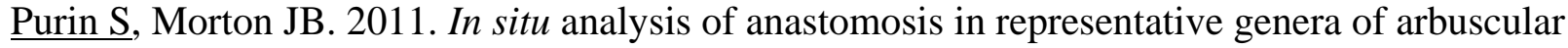
mycorrhizal fungi. Mycorrhiza. doi: 10.1007/s00572-010-0356-9.

Purin S, Rillig MC. 2008. Immuno-cytolocalization of glomalin in the mycelium of the arbuscular mycorrhizal fungus Glomus intraradices. Soil Biology and Biochemistry 40: 10001003. 
Purin S, Rillig MC. 2008. Parasitism of arbuscular mycorrhizal fungi: reviewing the evidence. FEMS Microbiology Letters 279: 8-14.

Purin S, Rillig MC. 2008. The arbuscular mycorrhizal fungal protein glomalin: Limitations, progress, and a new hypothesis for its function. Pedobiologia 51: 123-130.

Purin S, Klauberg Filho O, Sturmer SL. 2006. Mycorrhizae activity and diversity in conventional and organic apple orchards from Brazil. Soil Biology and Biochemistry 38: 1831-1839.

\section{Book Chapters}

Purin S, Klauberg Filho O. Glomalina: nova abordagem para entendermos a biologia dos fungos micorrízicos arbusculares. In: Siqueira, J.O.; Souza, F.A.; Cardoso, E.J.B.N.; Tsai, S.M. (Eds). Micorrizas: 30 anos de pesquisas no Brasil. Lavras: UFLA, 2010. 503-524.

\section{Oral Presentations}

In situ analysis of anastomosis in representative genera of Glomeromycotan fungi using a unique rhizohyphatron design.

$14^{\text {th }}$ Annual Davis College Graduate Research Day Conference, West Virginia University, Morgantown, WV, USA, 2010

In situ analysis of anastomosis in all genera of Glomeromycotan fungi using a unique rhizohyphatron design.

International Congress on Mycorrhizas (ICOM 6), Belo Horizonte, MG, Brasil, 2009

\section{Teaching experience}

\section{College level}

Guest Lecturer: "Beginner's Guide to Genetics" (GEN 101), 2008

West Virginia University, Morgantown, WV

Topic: Meiosis, mitosis and errors in genetic recombination"

Teaching assistant: "Soil Microbiology", 2004

Universidade do Estado de Santa Catarina, Lages, SC, Brasil

Topics: Concepts about microbial ecology, methods to assess diversity and activity of soil microbes, ecto and endomycorrhizal fungi.

\section{Junior high school}

Teacher: Science, chemistry and physics, 2006

Colégio Municipal de Indaial, Indaial, SC, Brasil 


\section{Awards}

Ph.D. scholarship from Fulbright/CAPES, with complete funding for 4 years.

First place, Ph.D. paper presentation. $14^{\text {th }}$ Annual Davis College Graduate Research Day Conference, West Virginia University, Morgantown, WV, USA, 2010

Second runner, oral presentation. International Congress on Mycorrhizas (ICOM 6), Belo Horizonte, MG, Brasil, 2009

Honorable mention, highest GPA in the graduating class, 2002

Universidade Regional de Blumenau, Blumenau, SC, Brasil

"Mérito Universitário Catarinense", 2001. The State government of SC (Brasil) selected 3 students with outstanding academic performance to be awarded with an international trip. Fulltime research activities were conducted at The University of Wisconsin Oshkosh, USA, for 30 days.

\section{Service}

Reviewer for the journals Mycologia, Symbiosis and Scientia Agricola

Reviewer of a proposal submitted to Fondecyt (Funding agency of the Chile government) - 2008

Member of the Search Committee for a faculty position in the Plant and Soil Sciences Division, West Virginia University, Morgantown, WV, 2010

Member of the Safety committee responsible for the South Agricultural Sciences Building Emergency Response Plan. West Virginia University, Morgantown, WV, since 2009

Part of the organizing committee of the National meeting on Soil Fertility, Plant Nutrition and Mycorrhizas (FERTBIO) in Brasil, 2004

\section{Memberships}

Gamma Sigma Delta Society, since 2009

International Mycorrhizal Society, since 2009 